\title{
Discovery of potent phosphodiesterase-9 inhibitors for the treatment of hepatic fibrosis
}

Yinuo Wu ${ }^{\mathrm{a}, \#}$, Quan Wanga, \#, Mei-Yan Jiang, \#, Yi-You Huang ${ }^{\mathrm{a}, \mathrm{b}}$, Ziran Zhu ${ }^{\mathrm{b}}$, Chuan Han ${ }^{\mathrm{a}}$, Yi-Jing Tian $^{\mathrm{a}}$, Bei Zhang ${ }^{\mathrm{a}}$, Hai-Bin Luo ${ }^{\mathrm{a}, \mathrm{b}, *}$

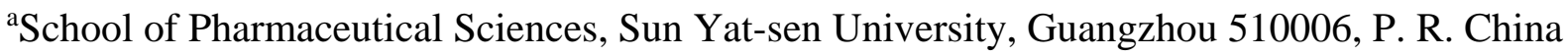

${ }^{\mathrm{b}}$ Key Laboratory of Tropical Biological Resources of Ministry of Education, School of Pharmaceutical Sciences, Hainan University, Haikou 570228, Hainan, China.

*To whom correspondence should be addressed. Fax: +86-20-39943000. E-mail: luohb77@mail.sysu.edu.cn or hbluo@hainanu.edu.cn

\section{Table of Contents:}

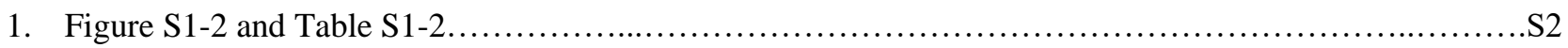

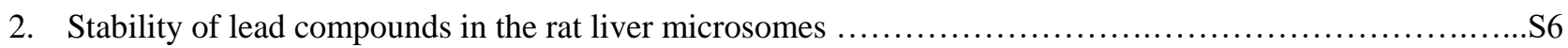

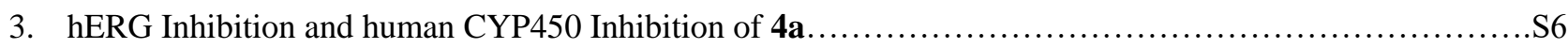

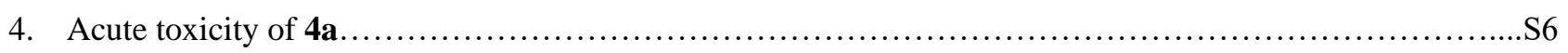

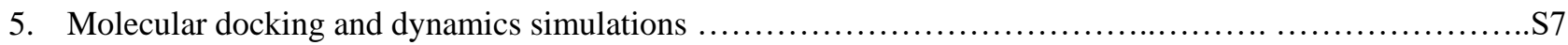

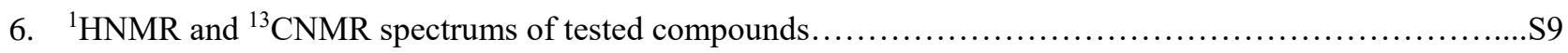

7. High-resolution mass spectra (HRMS) spectrums of tested compounds...............................S30

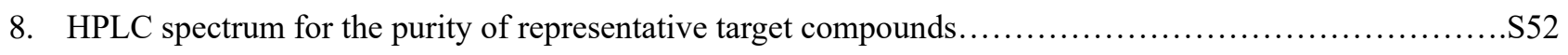

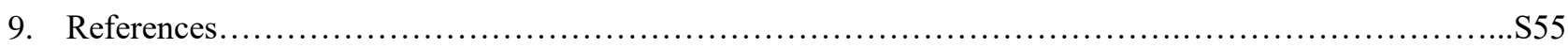


1. Figure S1-2 and Table S1

(A)

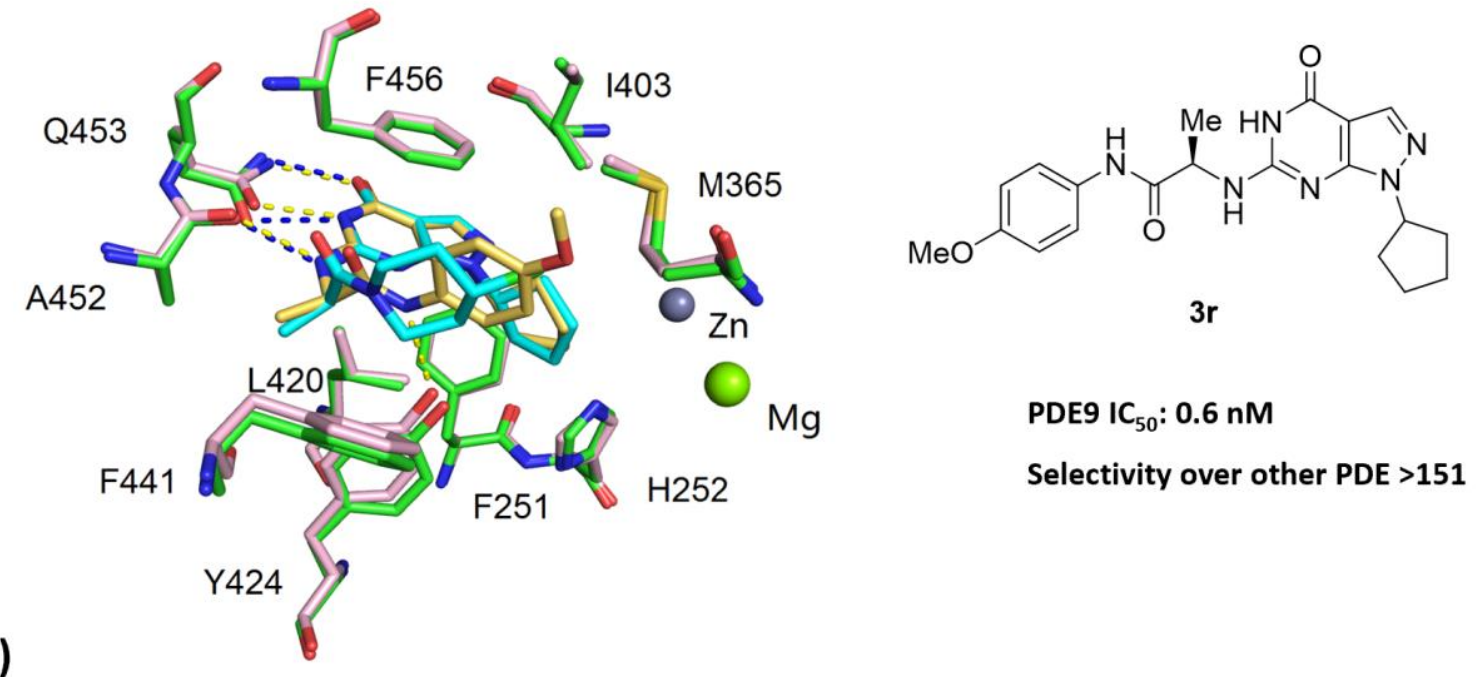

(B)
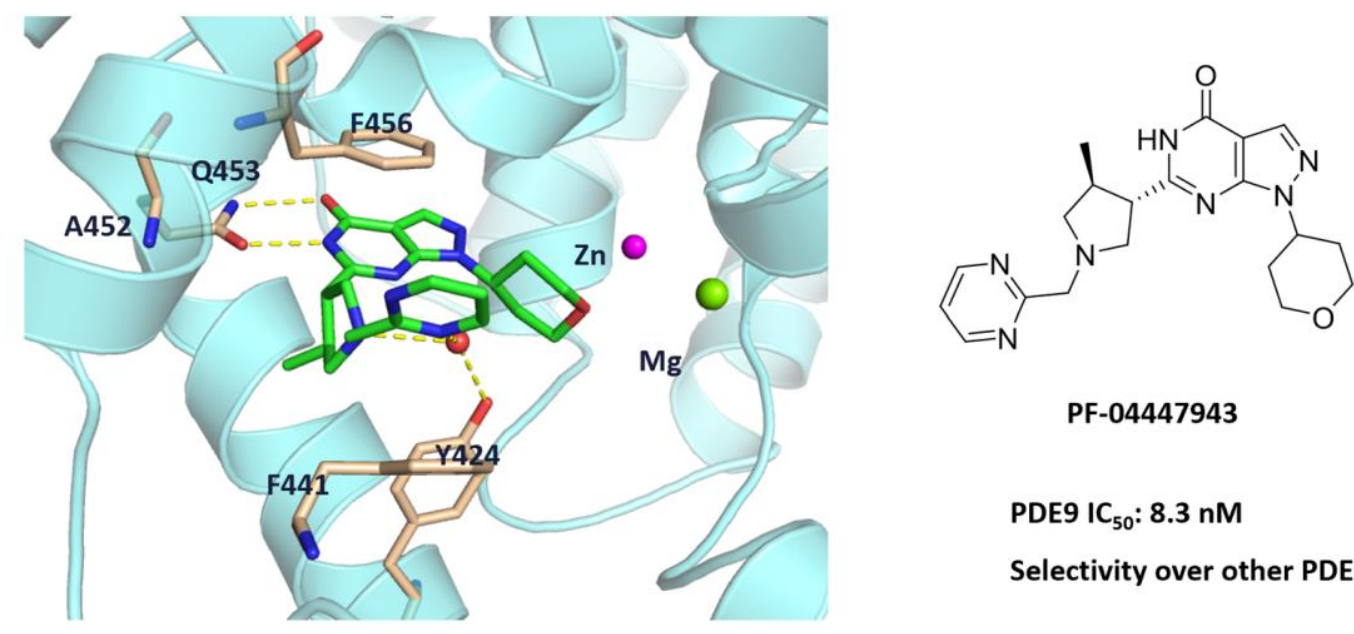

Selectivity over other PDE $>120$

Figure S1: (A) Superposition of PDE9-3a (PDB code: 6A3M, cyan and green) over PDE9-3r (PDB code: 4QGE, yellow and light pink). (B) The binding pattern of PDE9 in complex with compound PF04447943 (PDB code: 4E90). 

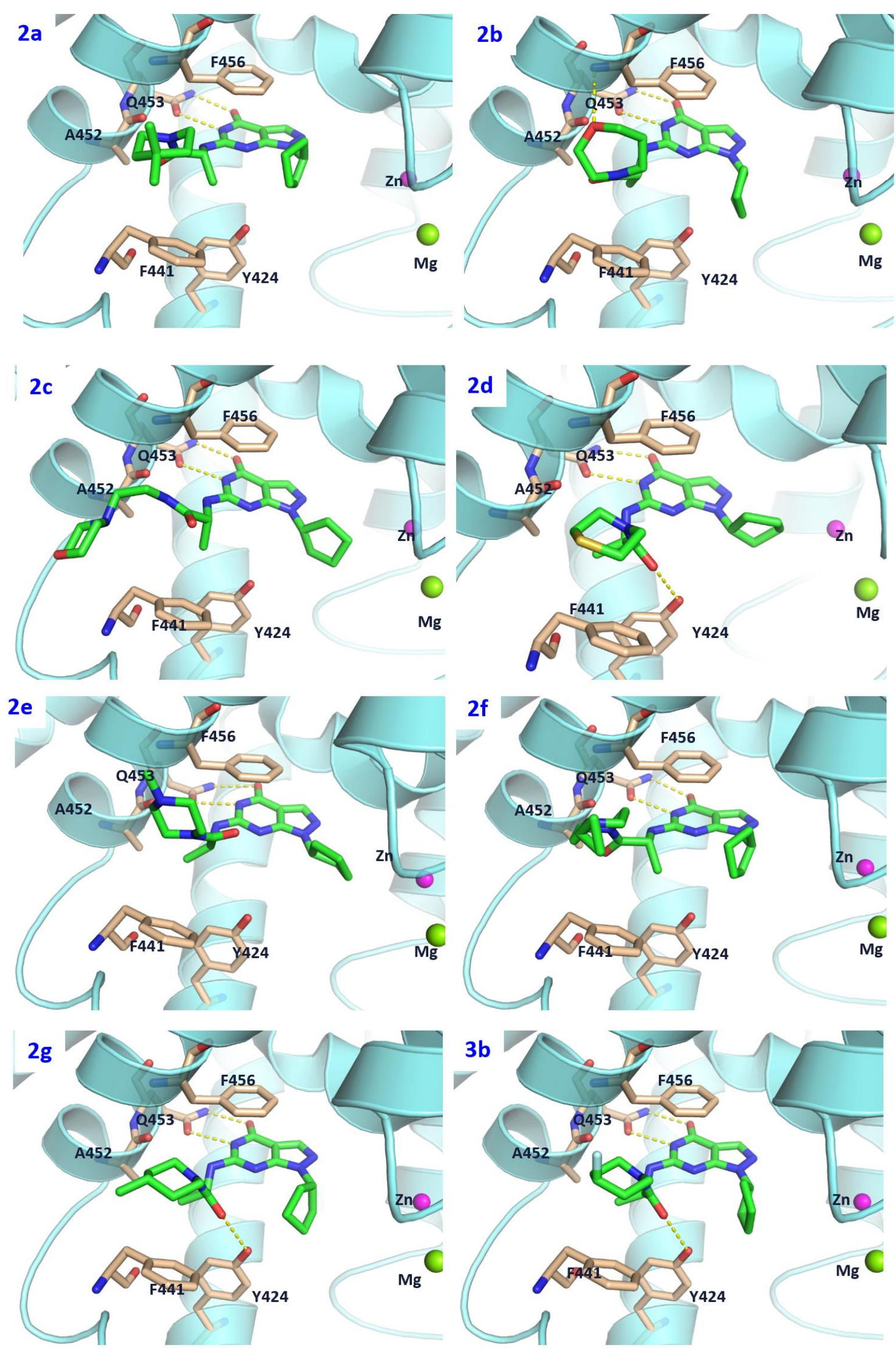


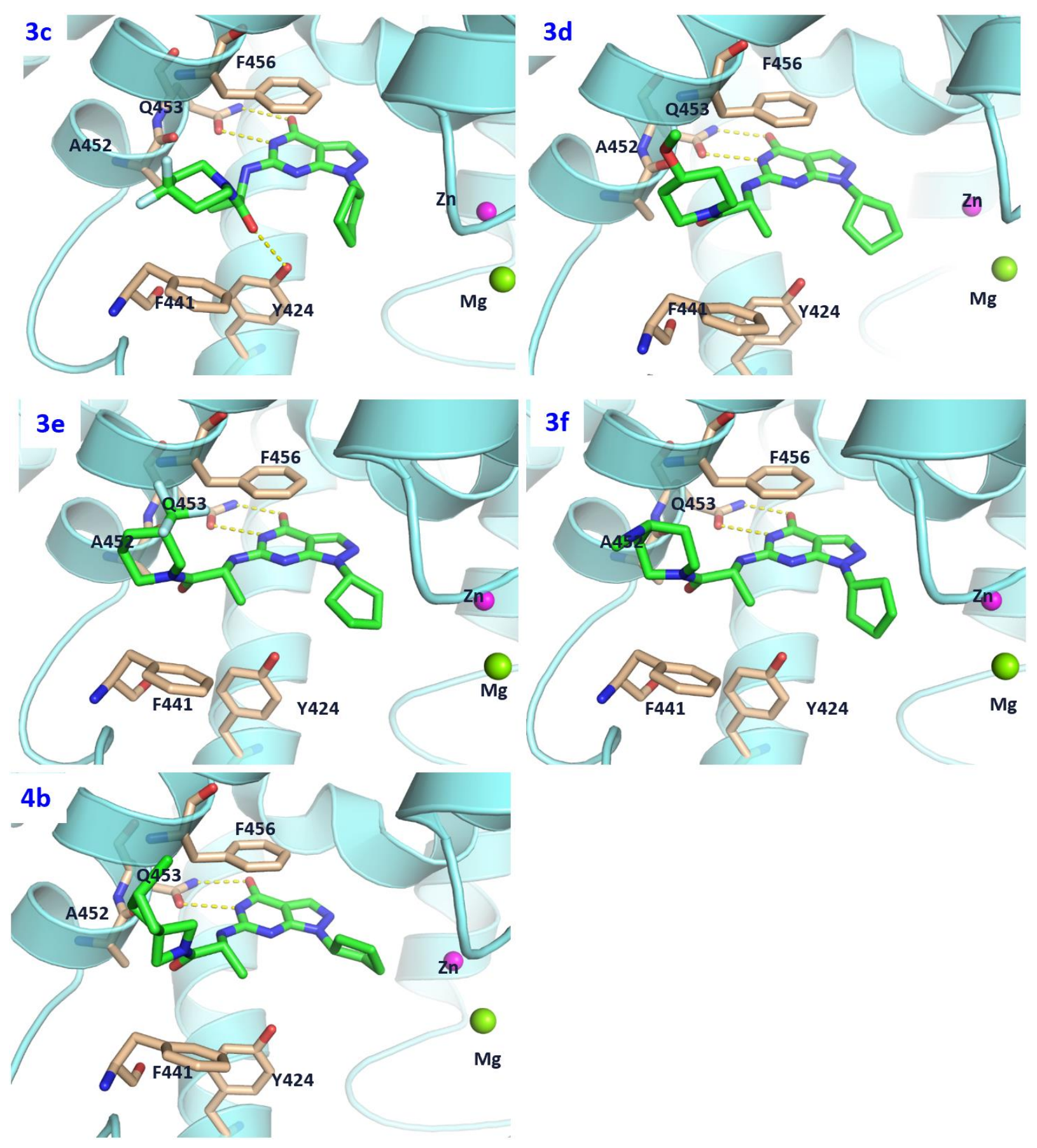

Figure S2. The predicted binding patterns between PDE9 and targeted compounds. 
Table S1: Statistics on diffraction data and structure refinement of the PDE9 complex with inhibitor 3a.

\begin{tabular}{|c|c|}
\hline Data collection & PDE9-3a \\
\hline Space group & $\mathrm{P} 4{ }_{1} 2{ }_{12}$ \\
\hline Unit cell $(\mathrm{a}=\mathrm{b}, \mathrm{c}, \AA)$ & $103.92,269.99$ \\
\hline Resolution $(\AA)$ & 2.7 \\
\hline Unique reflection & 41,252 \\
\hline Redundancy & $8.3(6.9)^{*}$ \\
\hline Completeness (\%) & $100(100)^{*}$ \\
\hline Average $\mathrm{I} / \sigma$ & $14.0(2.5)^{*}$ \\
\hline Rmerge & $0.130(0.698)^{*}$ \\
\hline \multicolumn{2}{|l|}{ Refinement } \\
\hline R-factor & 0.216 \\
\hline R-free & $0.253(4.8 \%) \S$ \\
\hline Resolution $(\AA)$ & 2.7 \\
\hline Reflections & 39,252 \\
\hline \multicolumn{2}{|l|}{ RMS deviation } \\
\hline Bond length $(\AA)$ & 0.008 \\
\hline Angle & $1.23^{\circ}$ \\
\hline \multicolumn{2}{|c|}{ Average B-factor $\left(\AA^{2}\right)$} \\
\hline Protein & $42.849(5322) \mathbb{I}$ \\
\hline inhibitor & $34.8(54) \mathbb{I}$ \\
\hline $\mathrm{Zn}$ & $50.6(2) \mathbb{I}$ \\
\hline $\mathrm{Mg}$ & $30.2(2) \mathbb{I}$ \\
\hline Water & $31.6(99) \mathbb{L}$ \\
\hline \multicolumn{2}{|c|}{ Ramachandran plot (\%) } \\
\hline Most favorable & 93.4 \\
\hline Allowed region & 6.4 \\
\hline General allowed & 0.2 \\
\hline
\end{tabular}

*the numbers in parentheses are for the highest resolution shells of 2.7-2.8 $\AA$ for PDE9-3a.

$\S$ The percentage of reflection omitted in the refinement.

I The numbers in the parentheses are the numbers of atoms. 


\section{Stability of Lead Compounds in the Rat Liver Microsomes.}

The assays were performed at the Medicilon Company, Shanghai, China. The experimental procedures were similar to those in our previous study. ${ }^{1}$ Lead compounds was dissolved in $100 \%$ DMSO to prepare a $0.5 \mathrm{mM}$ stock solution and diluted to a final concentration of $1.5 \mu \mathrm{M}$ for the experiments. sildenafil was used as the positive control.

\section{Biopharmaceutical Profiling (hERG Inhibition and Human CYP450 Inhibition).}

The assays were performed by the Medicilon Company, Shanghai, China. hERG inhibition was performed using an automated patch clamp electrophysiology measurement in CHO-hERG cells. ${ }^{1}$ The CYP450 inhibition assay was performed by incubating compound $\mathbf{4 a}$ with human liver microsomes and NADPH in the presence of the CYP450-isoform specific probe substrate. The IC50 values of compound $\mathbf{4 a}$ for five CYP isoenzymes (i.e., CYP1A2, CYP2B6, CYP2C9, CYP2D6, and CYP3A4) were determined.

\section{Acute Toxicity of Compound 4a.}

The acute toxicity was tested according to similar protocols that were described in our previous study. ${ }^{1}$ Thirty KM mice (22 days, $18-20$ g), which were purchased from the Laboratory Animal Center of Sun Yat-Sen University (Guangzhou, China), were used to evaluate the acute toxicity of 4a. Mice were randomly divided into three groups, and each group was given in single oral dose of 0,1000 , or $1500 \mathrm{mg} / \mathrm{kg} \mathbf{4 a}$ on the first day of the experiment. Mice were maintained on a $12 \mathrm{~h} \mathrm{light/dark} \mathrm{cycle}$ (light from 7:00 to 19:00) at room temperature and 60-70\% relative humidity. Sterile food and water were provided according the institutional guidelines. Prior to each experiment, mice were fasted 
overnight and allowed free access to water. Compound $4 \mathbf{a}$ was dissolved in 5\% DMSO/10\% Solutol/85\% water solution and orally administered. Mice were observed for any abnormal behavior and mortality and weighed $4 \mathrm{~h}$ after $4 \mathrm{a}$ was administered and then every $24 \mathrm{~h}$ for 14 days. Animals were sacrificed on the 14th day, and tissue samples of the heart, liver, and kidney were macroscopically examined for possible damage.

\section{Molecular Docking and Molecular Dynamics Simulations.}

The X-ray crystal structure of 28s-PDE9A complex (PDB ID: 4GH6) reported in our previous studies $^{2}$ was selected for molecular modeling, and Surflex-dock embedded in the software Tripos Sybyl 2.0 was used. Two metal ions crucial for the PDE's catalytic activity in the catalytic domain and water molecules coordinate these two metal ions were retained. Hydrogen atoms were added, and the ionizable residues were protonated at the neutral $\mathrm{pH}$. The protomol, was generated using the parameters by default. The parameters of proto_thresh and proto_bloat were assigned 0.5 and 0 , respectively. After the protomol was the prepared, molecular docking was performed for test molecules.

After molecular docking completed, similar MD simulation procedures as previous studies were used to equilibrate the whole system. Here $8 \mathrm{~ns}$ MD simulations were carried out in the NPT ensemble with a constant pressure of $1 \mathrm{~atm}$ and a constant temperature of $300 \mathrm{~K}$. The periodic boundary conditions were adopted, along with an $8 \AA$ cutoff for long-range electrostatic interactions with the partial mesh Ewald (PME) method. The SHAKE algorithm was utilized to deal with all bonds involving hydrogen atoms, and hence the time step was set to 2 fs. An Intel Xeon E5620 CPU and an NVIDIA Tesla C2050 GPU, which are available in performing floating-point calculations, were applied to accelerate the process of MD simulations for each system. Subsequently, the 100 snapshots were isolated from the final $1.0 \mathrm{~ns}$ period of the MD simulation trajectories, and then used for binding- 
free-energy calculations by the MM-PBSA approach. For the electrostatic contribution to the solvation-free energy, the PBSA program in the Amber 16 suite was used, which could numerically solves the Poissone Boltzmann equations.

In light of the MM-PBSA method, the binding free energy ( $\Delta G$ bind) can be calculated by the

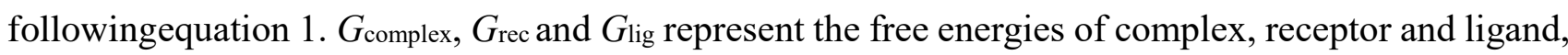
respectively.

$$
\Delta G_{\text {bind }}=G_{\text {complex }}-G_{\text {rec- }}-G_{\text {lig }}
$$

Each free energy was evaluated by the sum of the MM energy $E_{\mathrm{MM}}$, the solvation free energy $G_{\text {solv, }}$, and the entropy contribution $S$, respectively, leading to equation 2 .

$\Delta G_{\text {bind }}=\Delta E_{\mathrm{MM}}+\Delta G_{\text {solv }}-T \Delta S$

$\Delta E_{\mathrm{MM}}$ is the gas phase interaction energy and can be decomposed into $E_{\mathrm{MM}}$, comp, $E_{\mathrm{MM}}$, rec and $E_{\mathrm{MM}}$, lig. Solvation free energy is evaluated by the sum of the electrostatic solvation free energy and nonpolar solvation free energy. The electrostatic solvation free energy, $\Delta G_{\mathrm{PB}}$, was solved by the Poisson Boltzmann (PB) equation, while the nonpolar solvation free energy varies proportionally with the solvation accessible surface area (SASA), leading to by equation 3 and 4 .

$$
\begin{aligned}
& \Delta G_{\mathrm{solv}}=\Delta G_{\mathrm{PB}}+\Delta G_{\mathrm{np}} \\
& \Delta G_{\mathrm{np}}=\gamma \mathrm{SASA}+b
\end{aligned}
$$

In order to get the compromise between efficiency and accuracy, entropy contribution term was omitted for $\Delta G$ bind in equation 2 , since the calculations of the entropy contribution is extremely timeconsuming for large protein-ligand systems. The default parameters were adopted, with $\gamma=0.0072$ $\mathrm{kcal} /\left(\AA^{2}\right)$ and $b=0 \mathrm{kcal} / \mathrm{mol}$.The charges of $\mathrm{Mg}^{2+}$ and $\mathrm{Zn}^{2+}$ were assigned 2.0 for PB calculations, and their bond raddi were used for SA calculations. 
6. ${ }^{1} \mathrm{H}$ NMR and ${ }^{13} \mathrm{C}$ NMR spectrum.
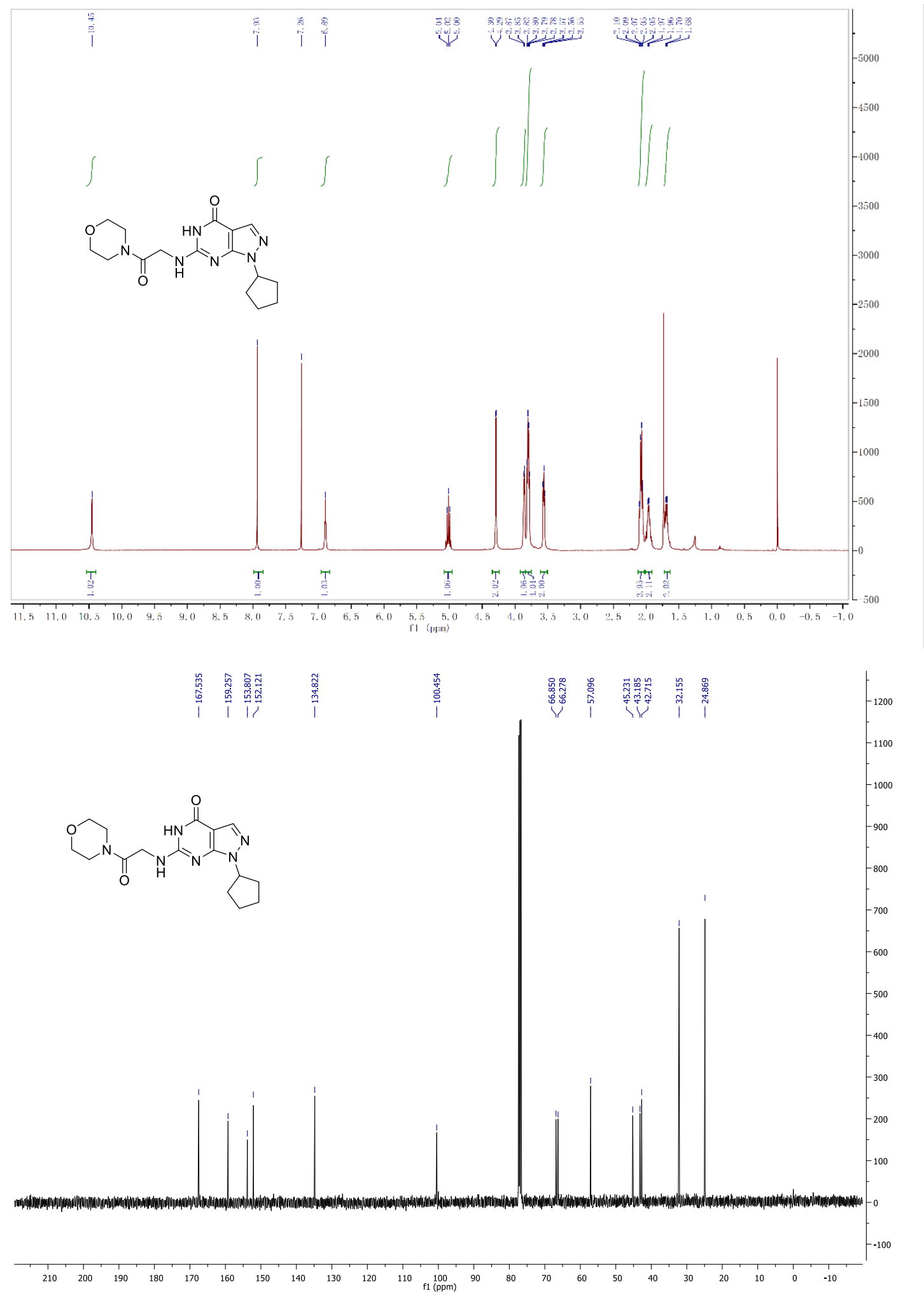

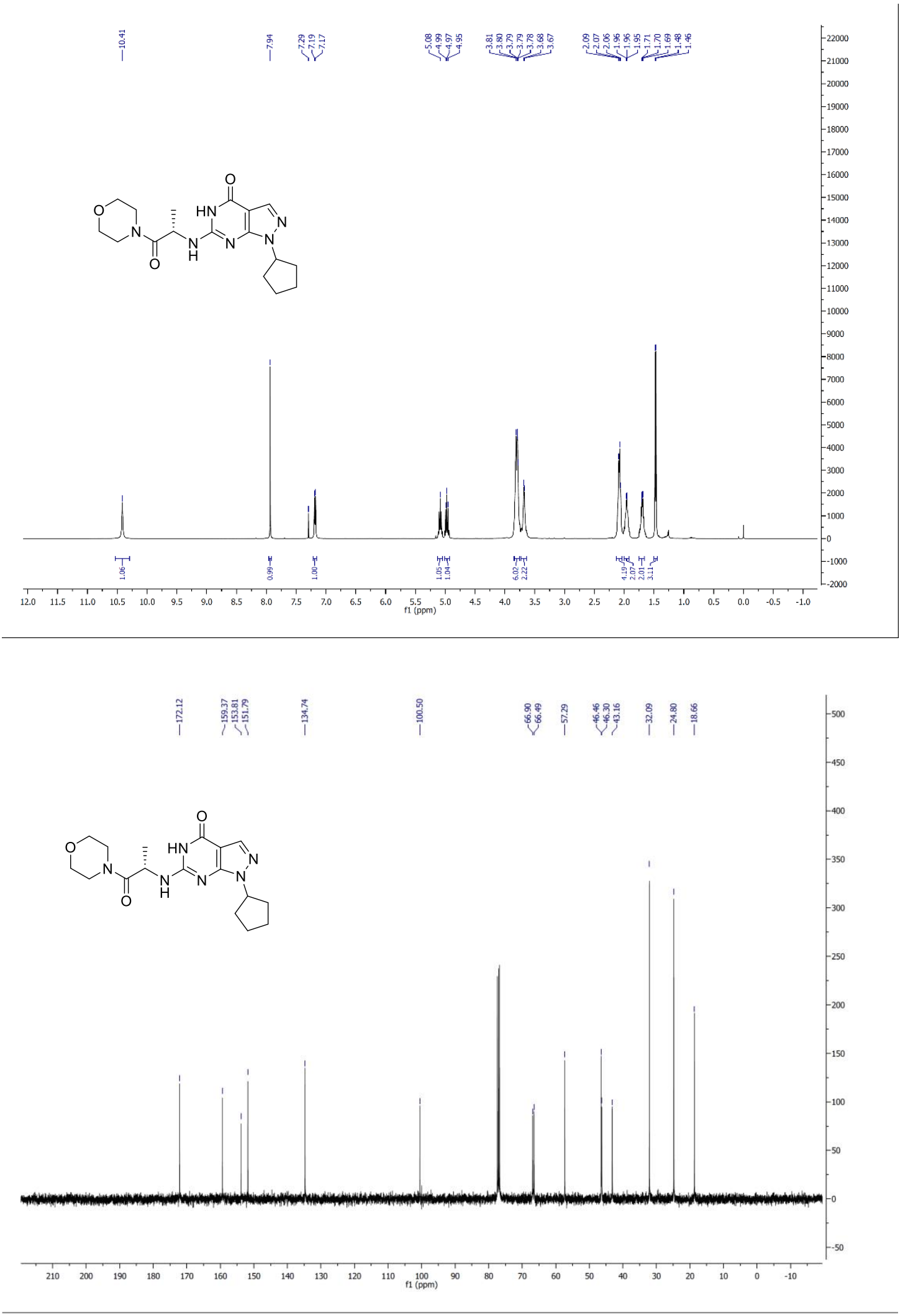


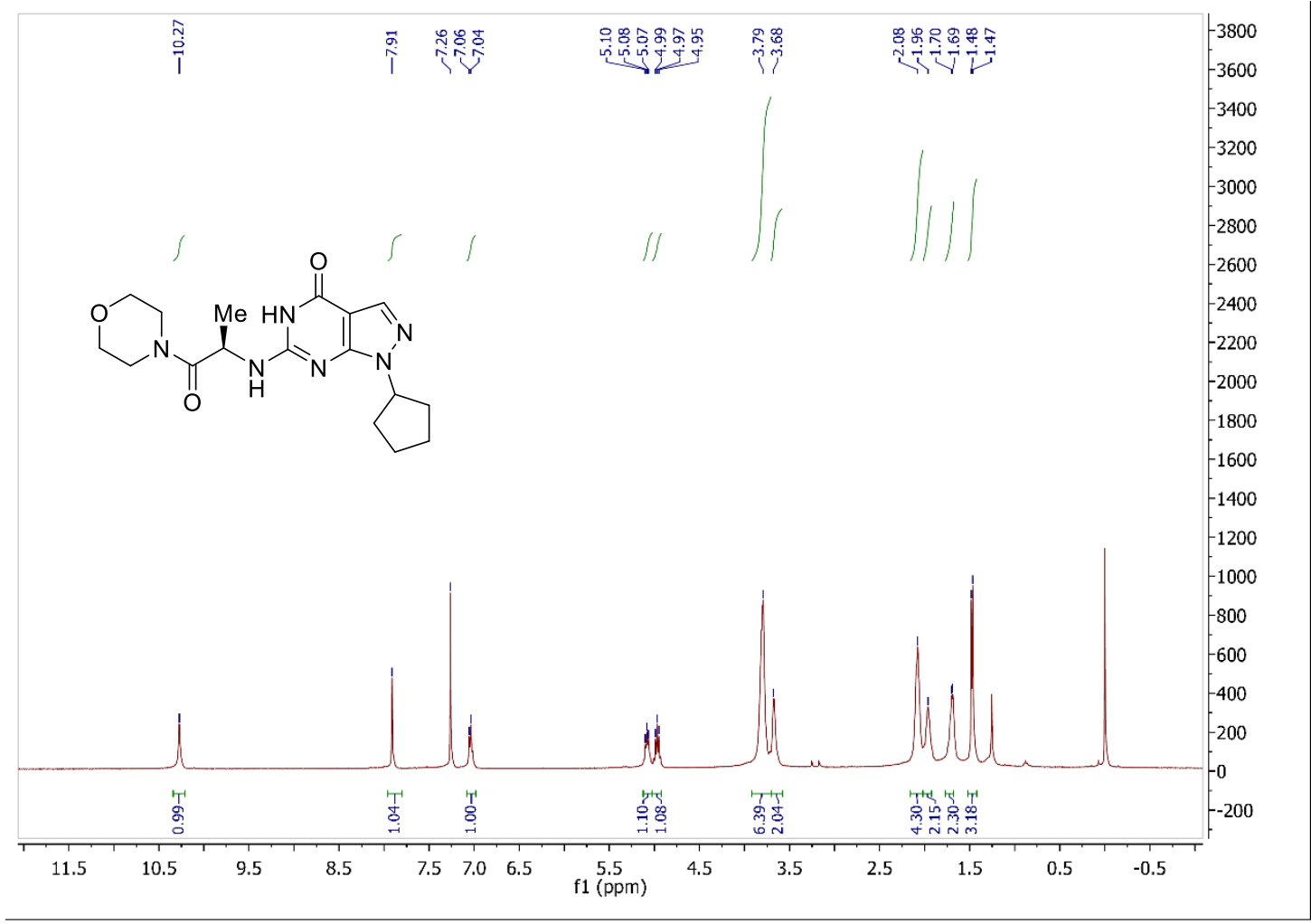

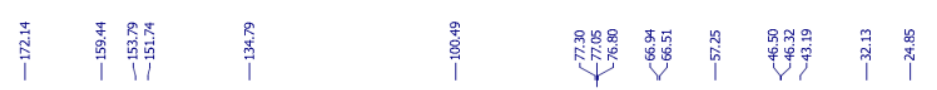
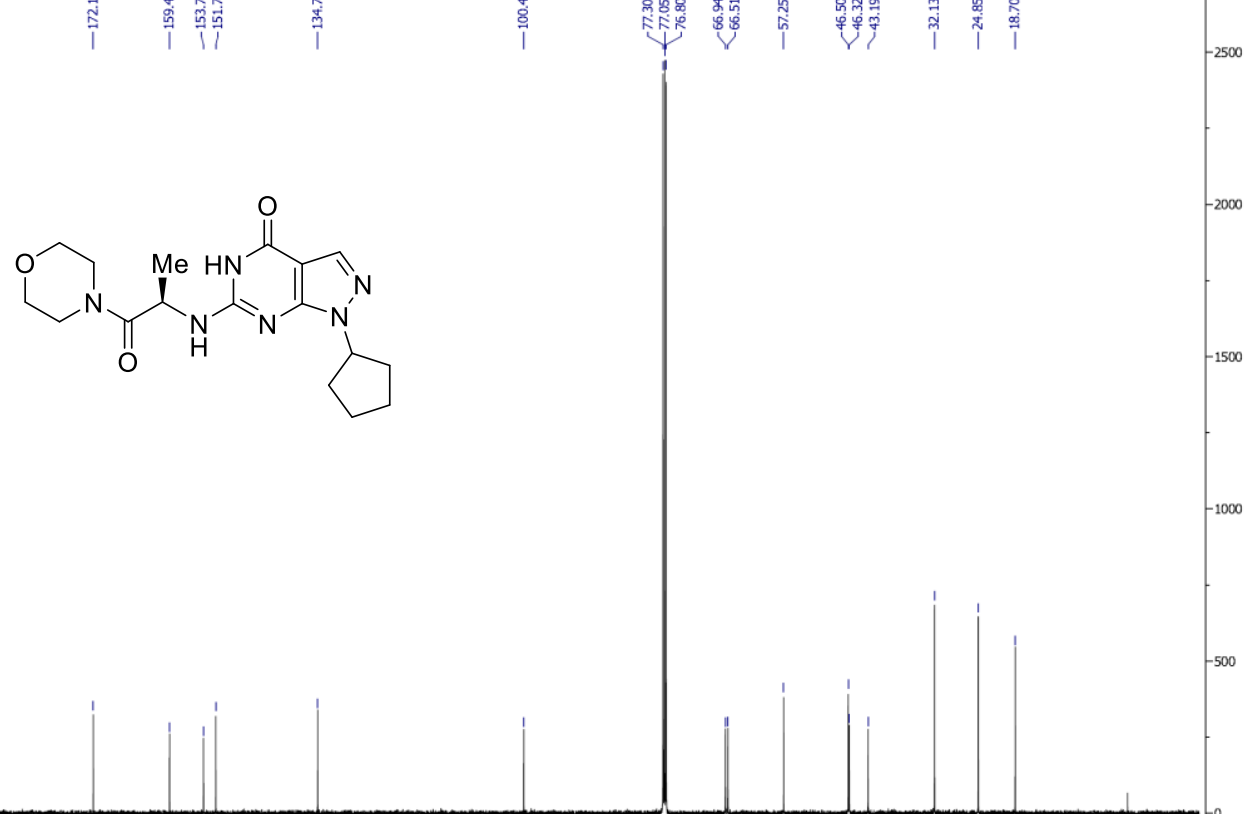

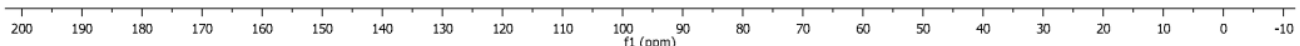




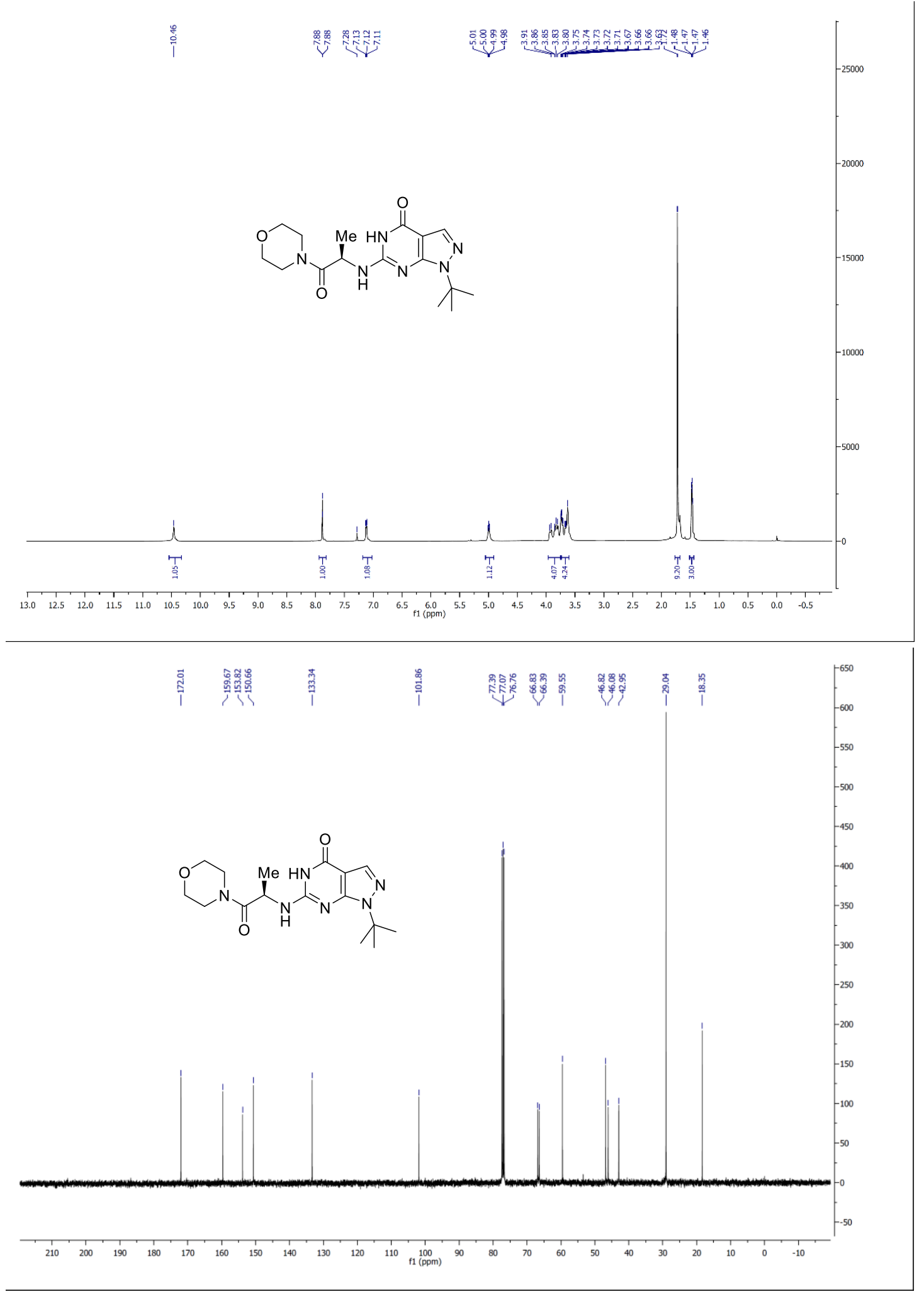




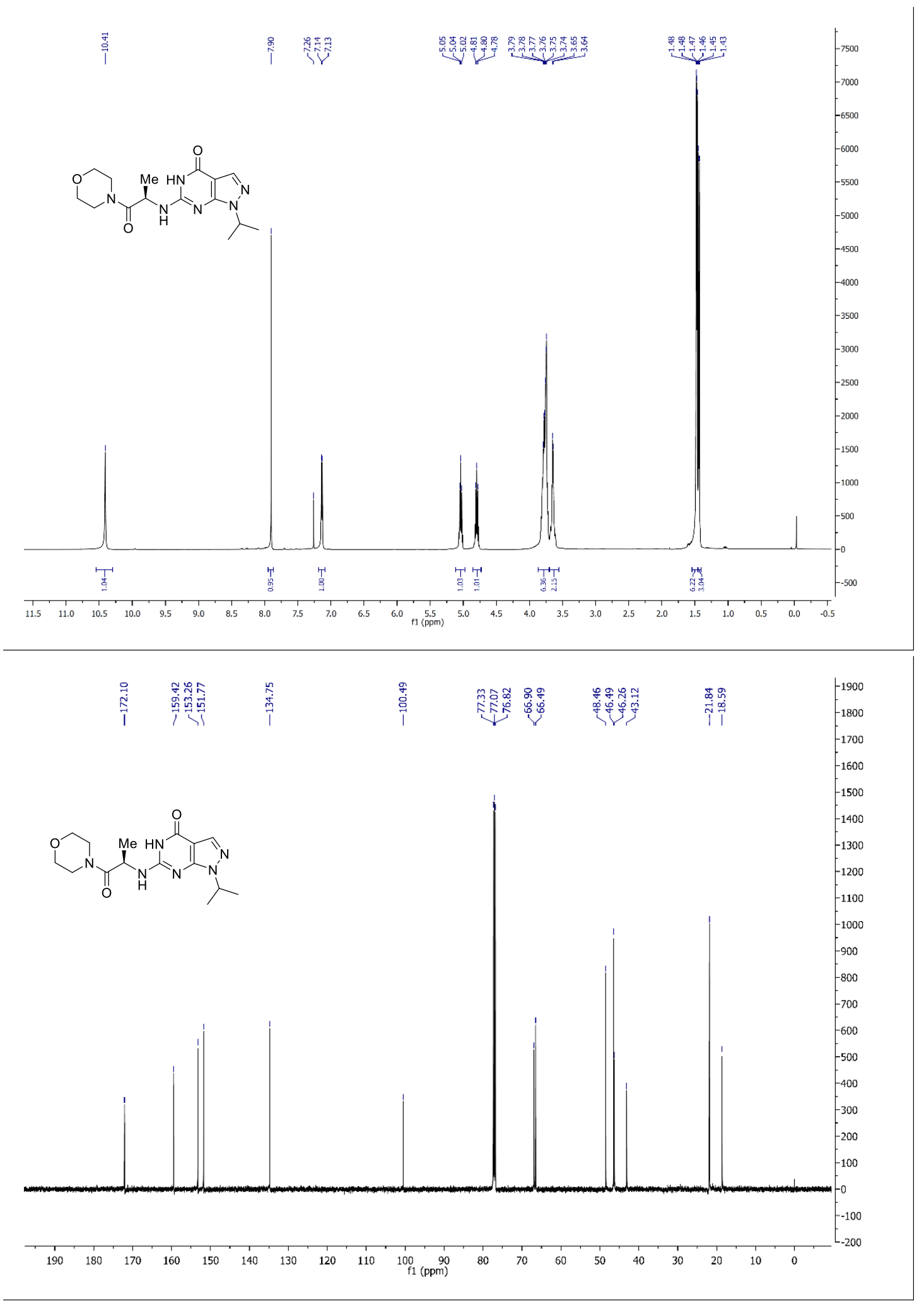



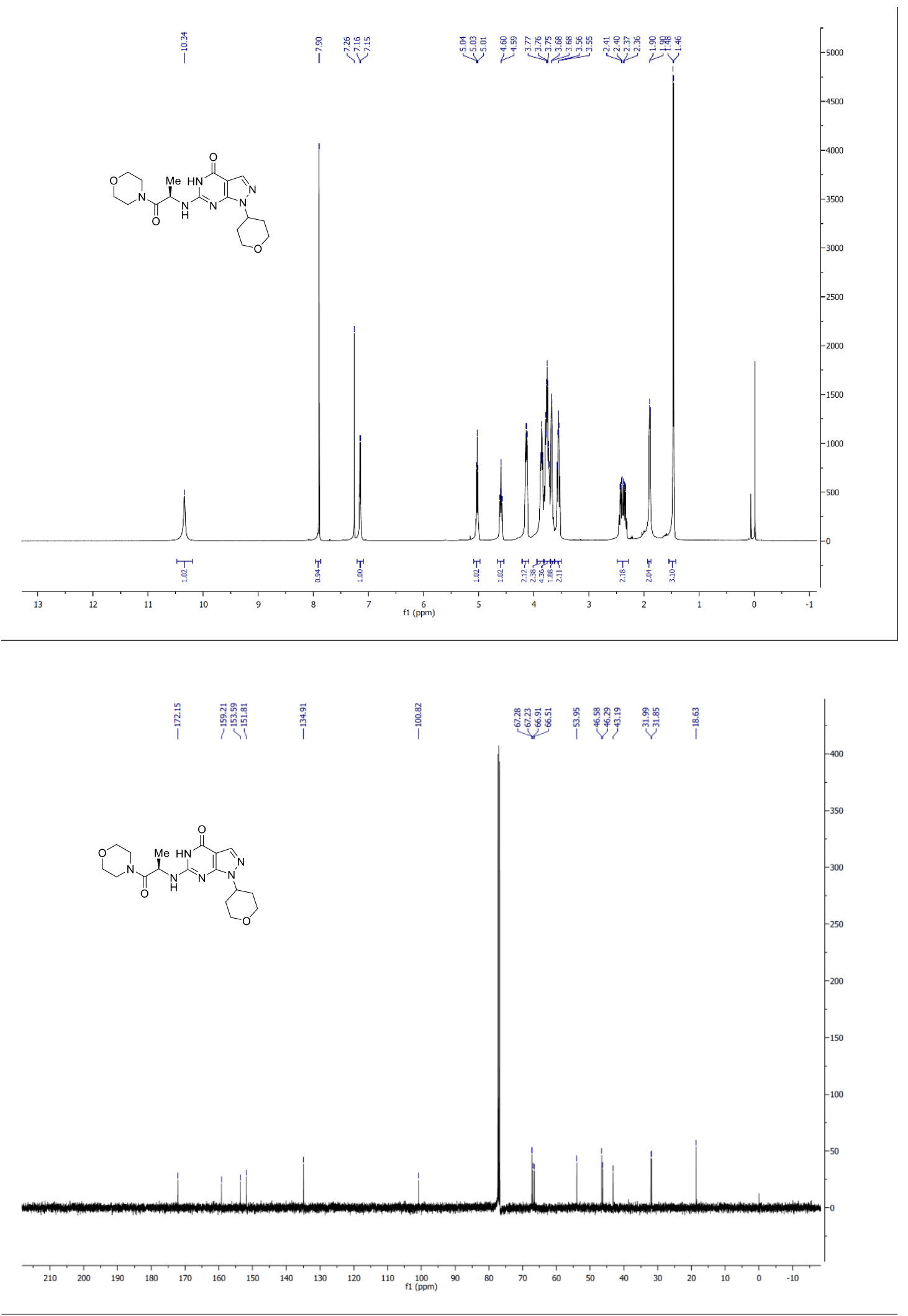

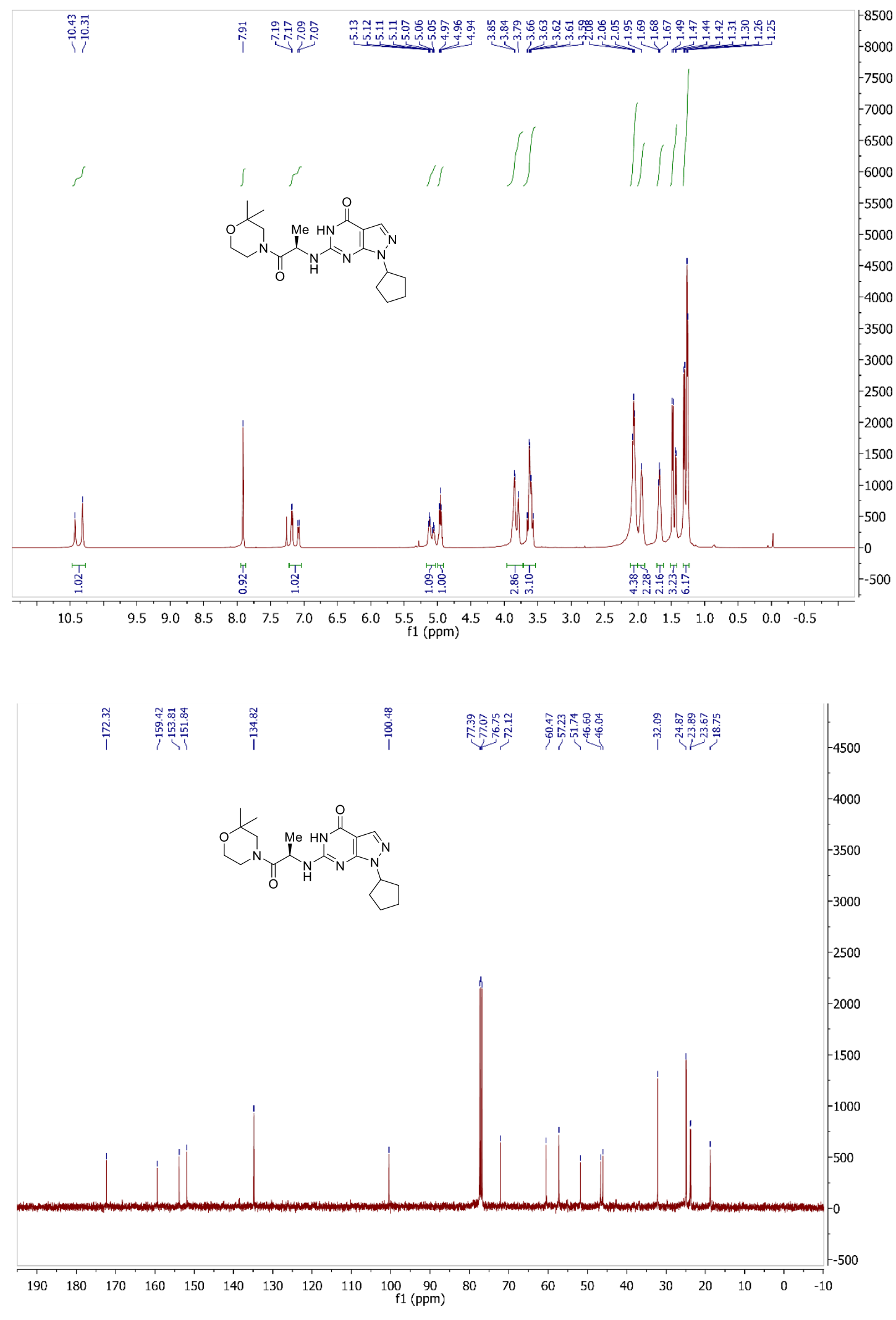

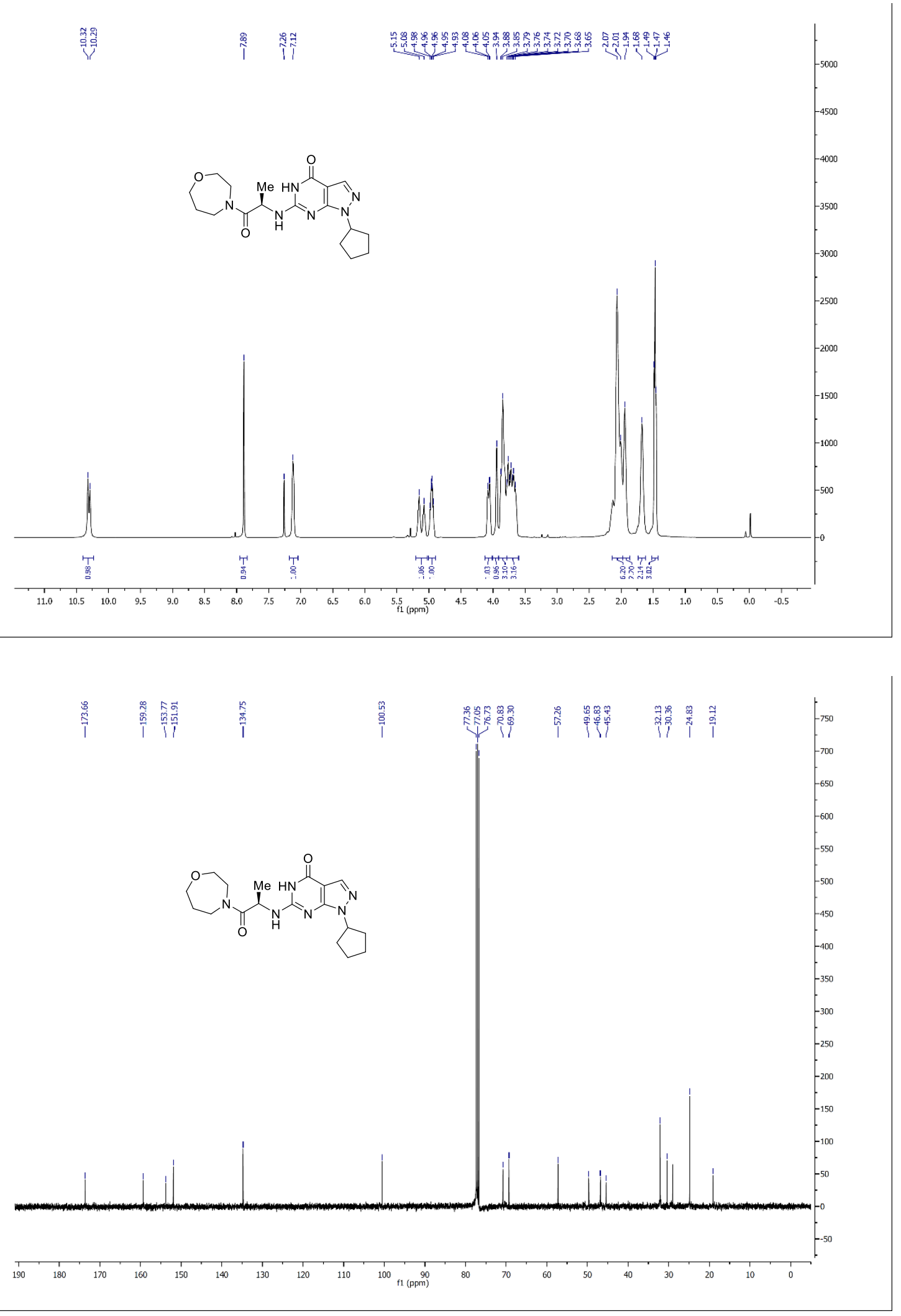


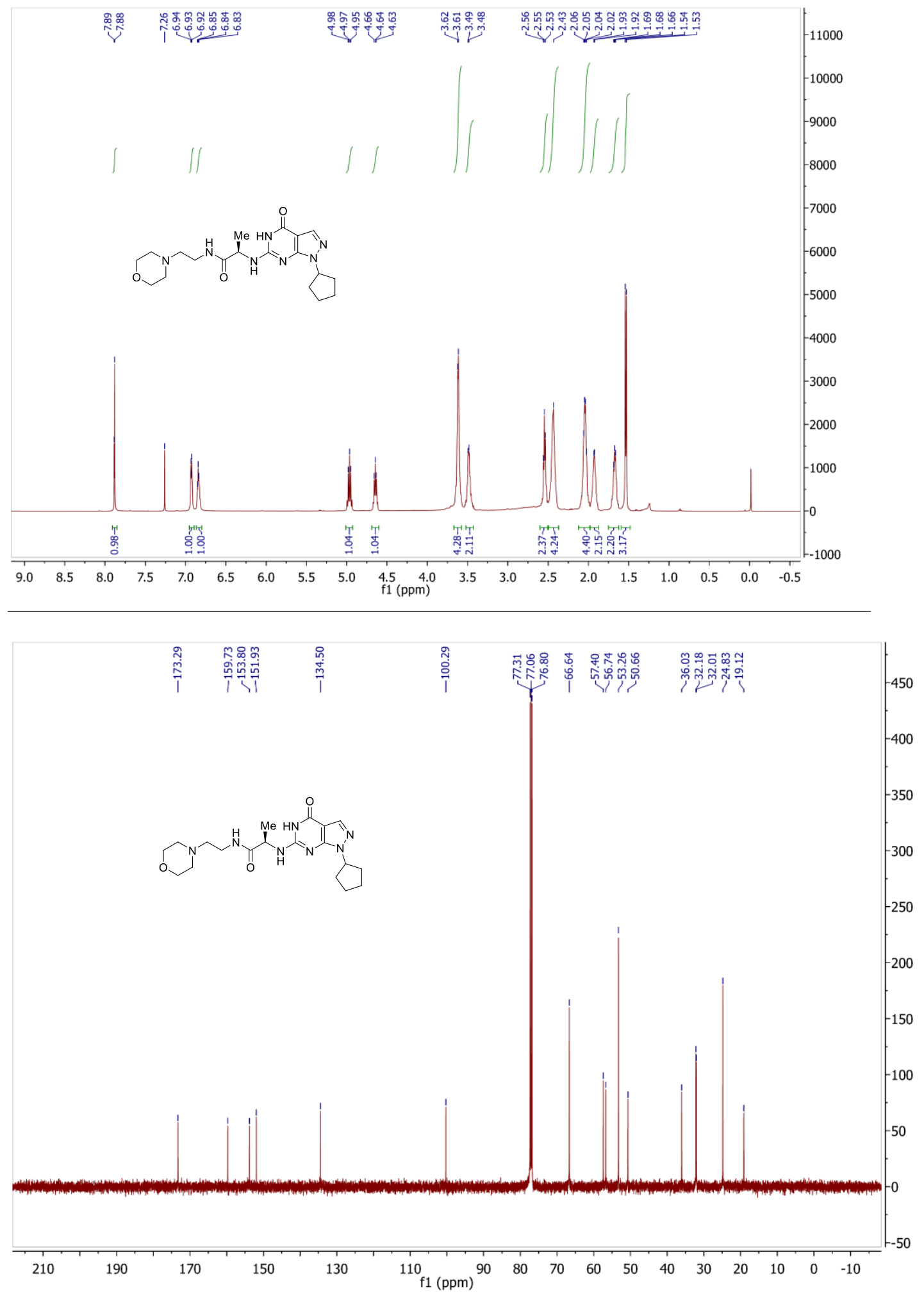



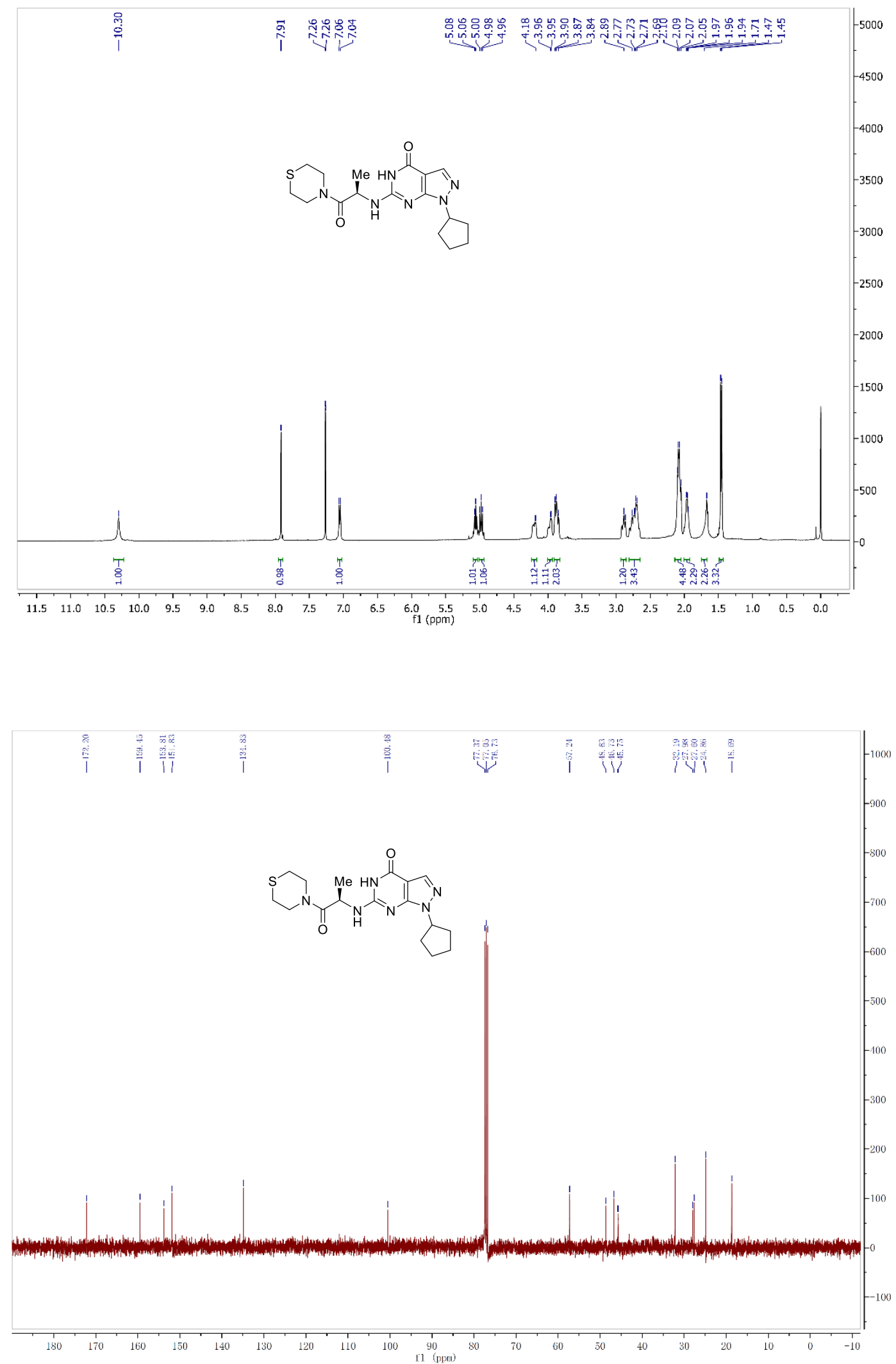

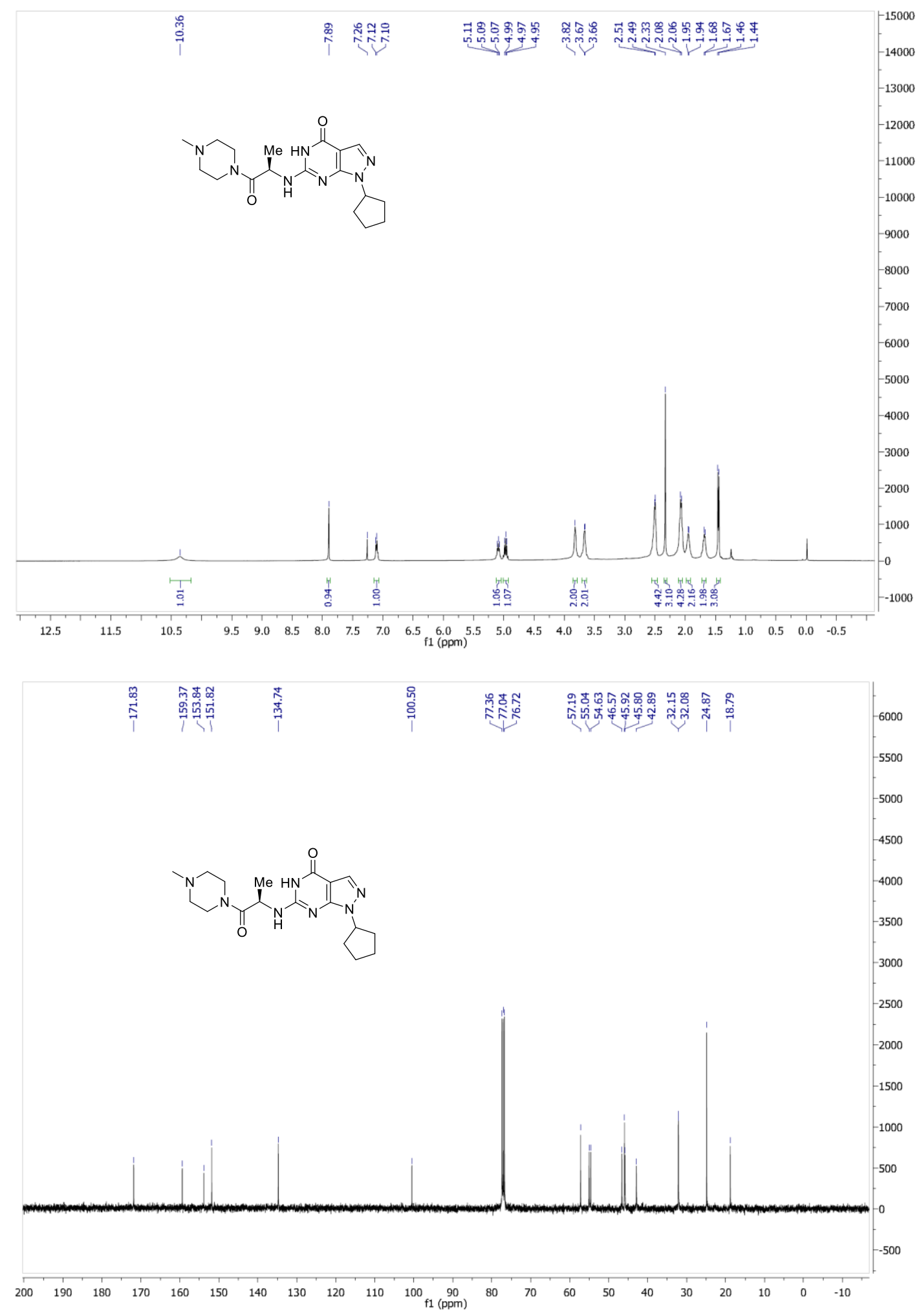

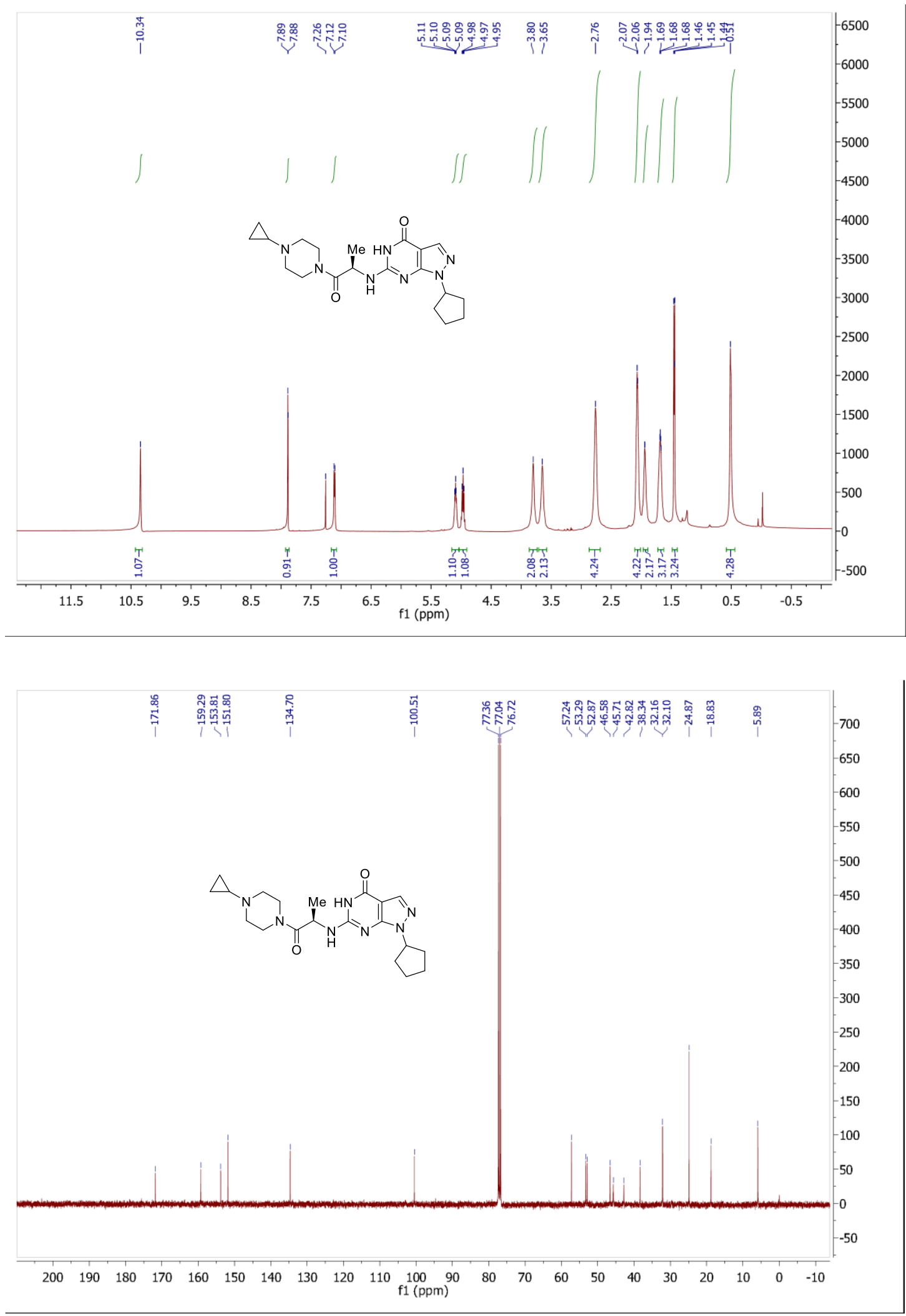


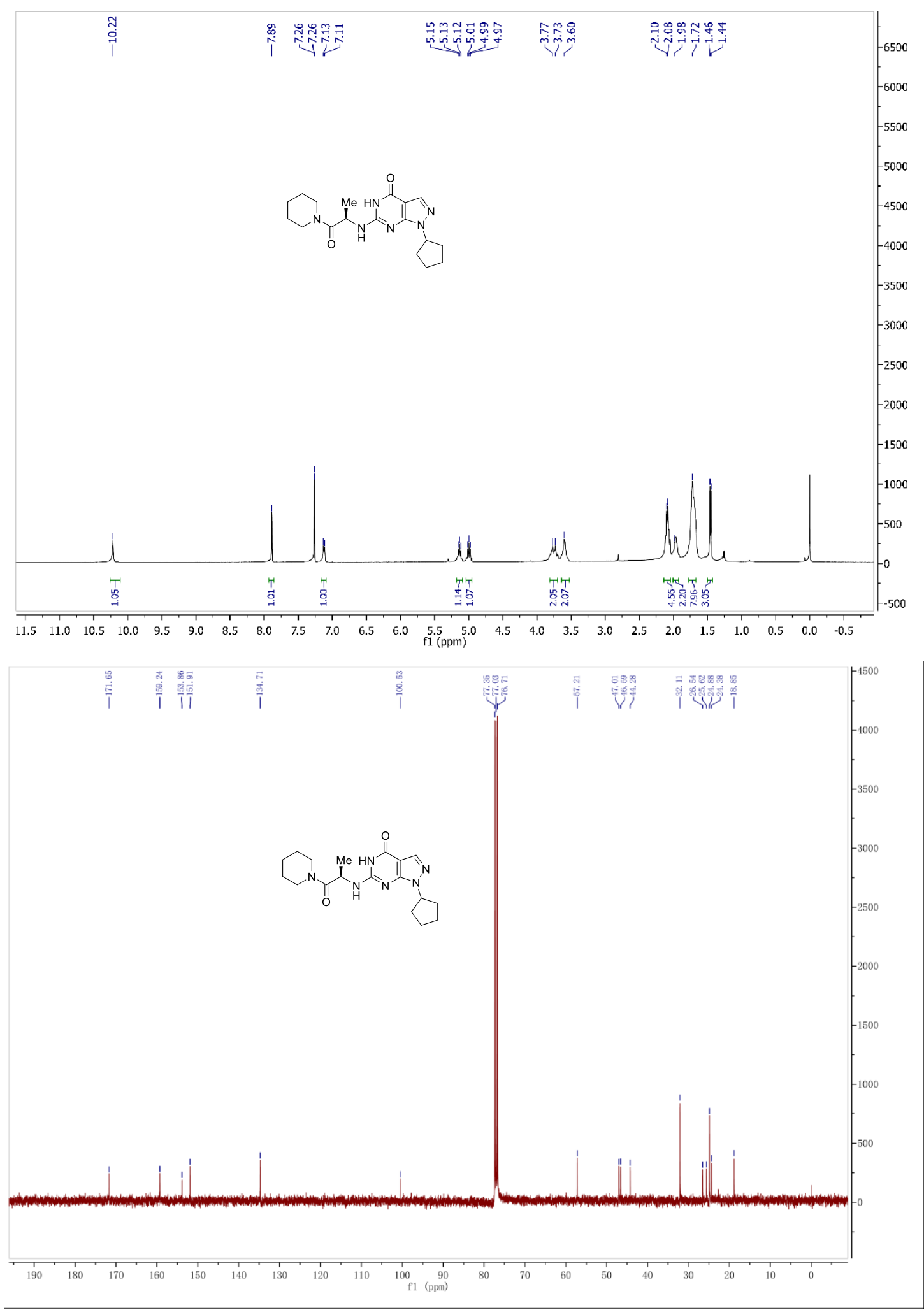




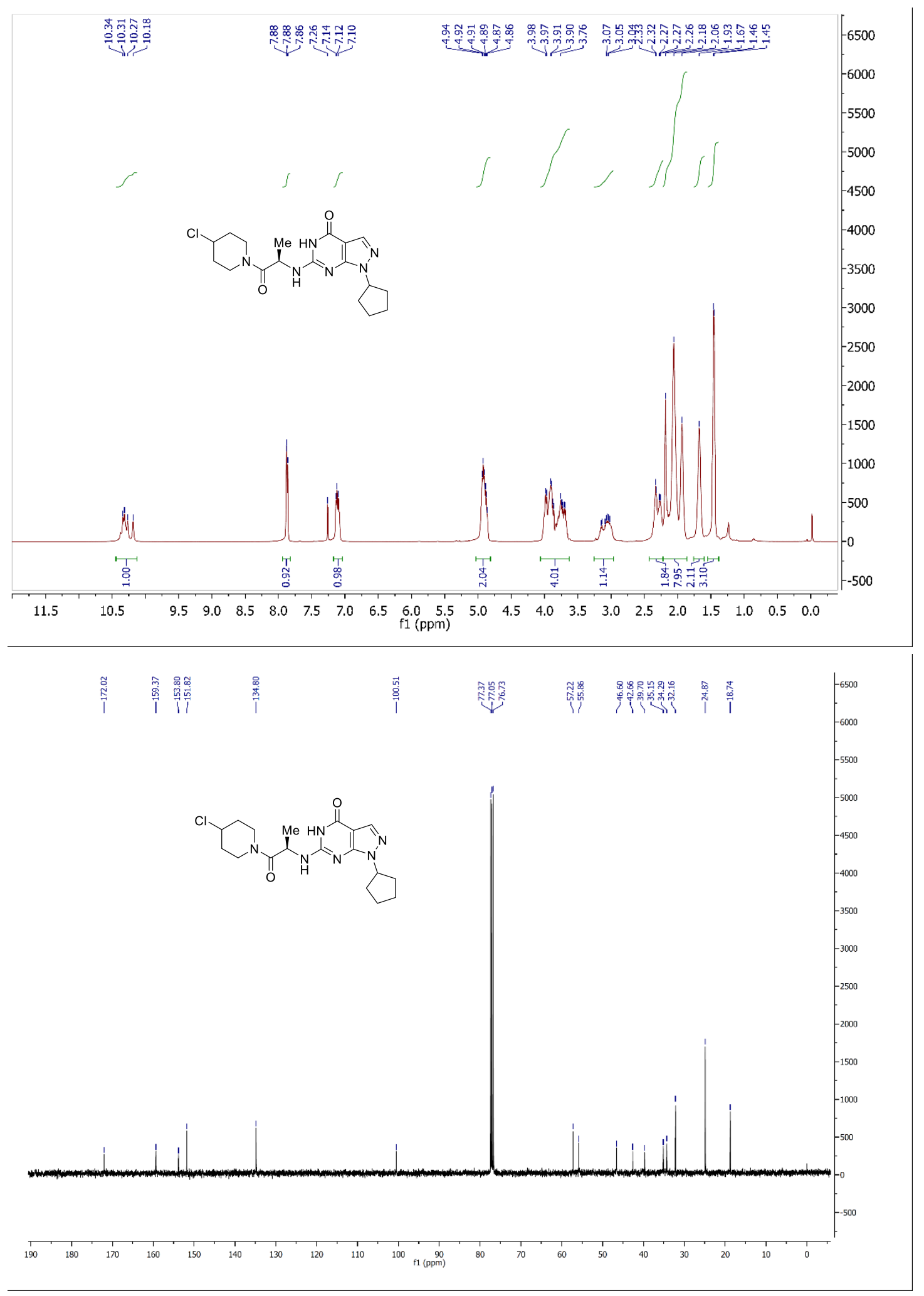




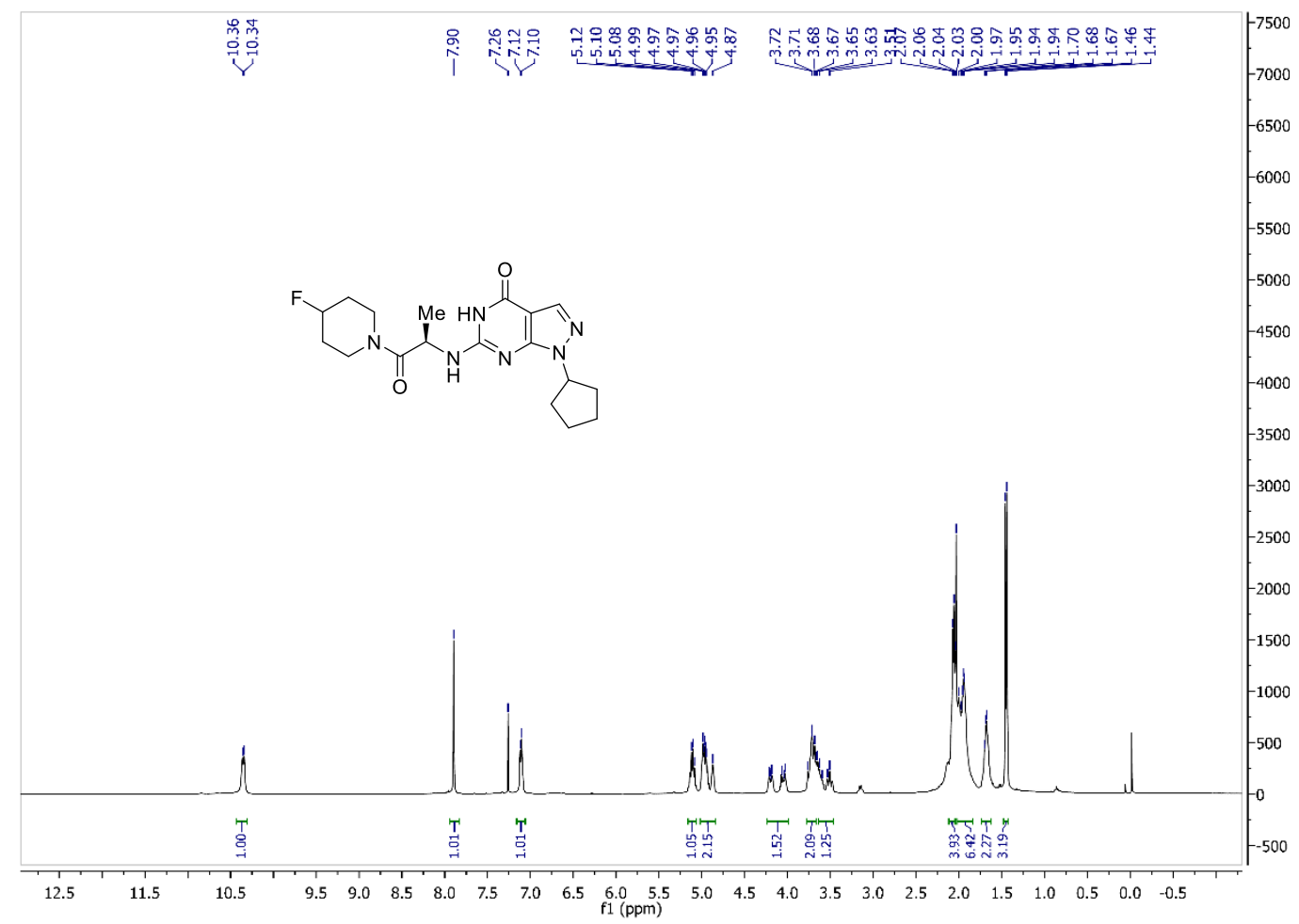

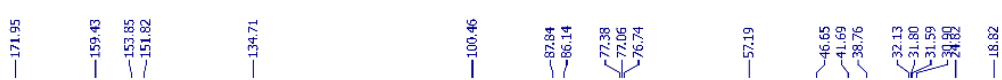

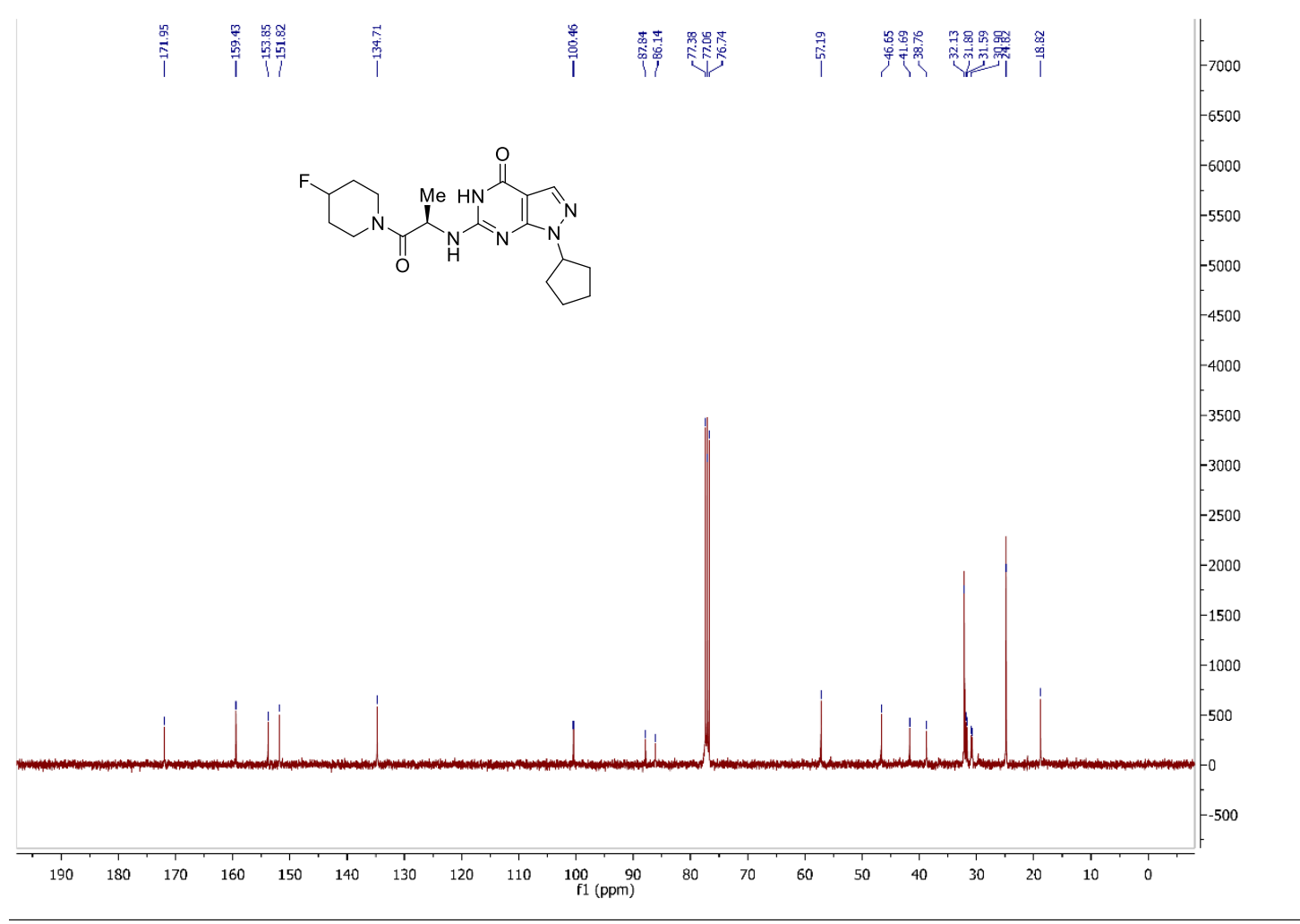




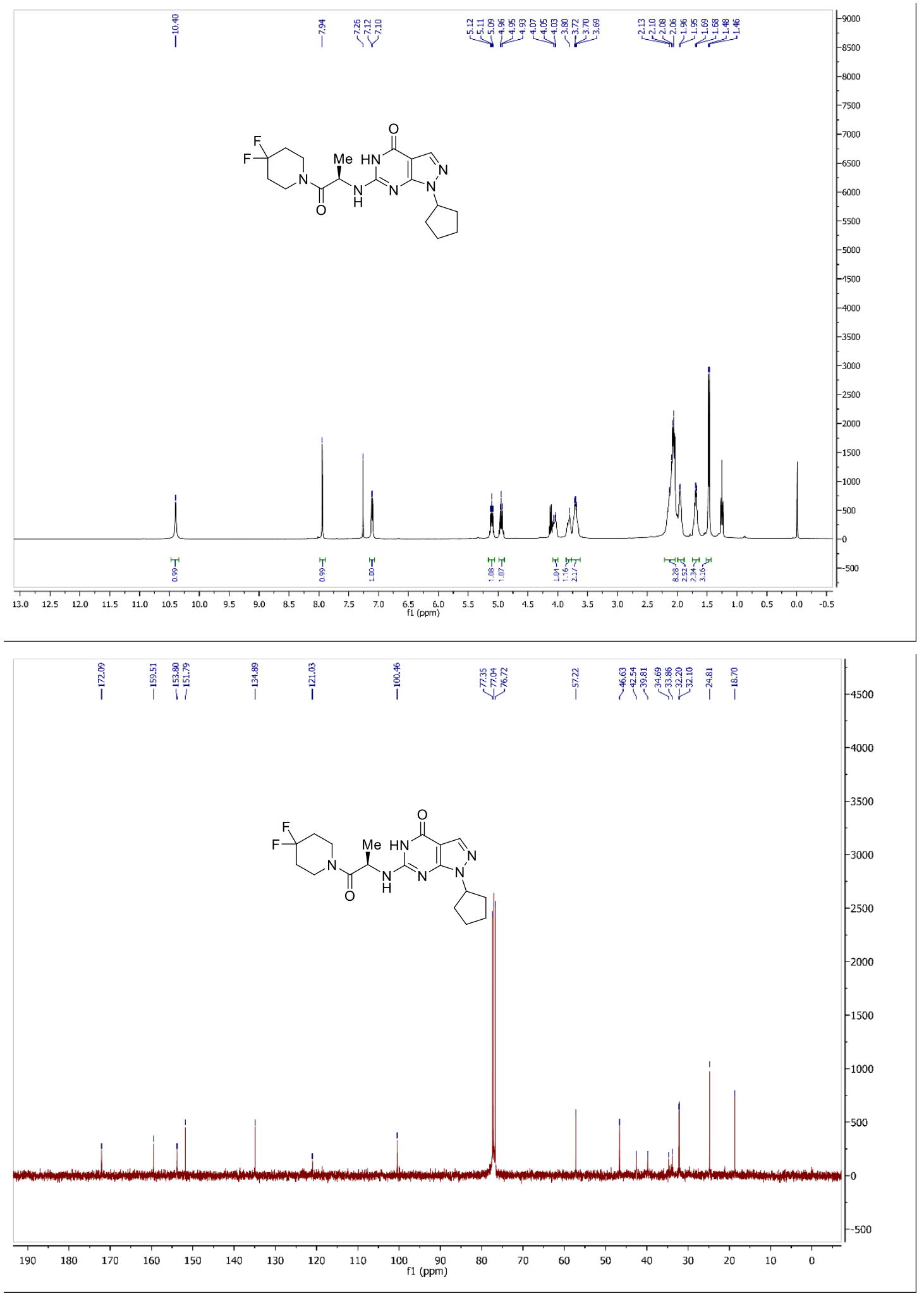



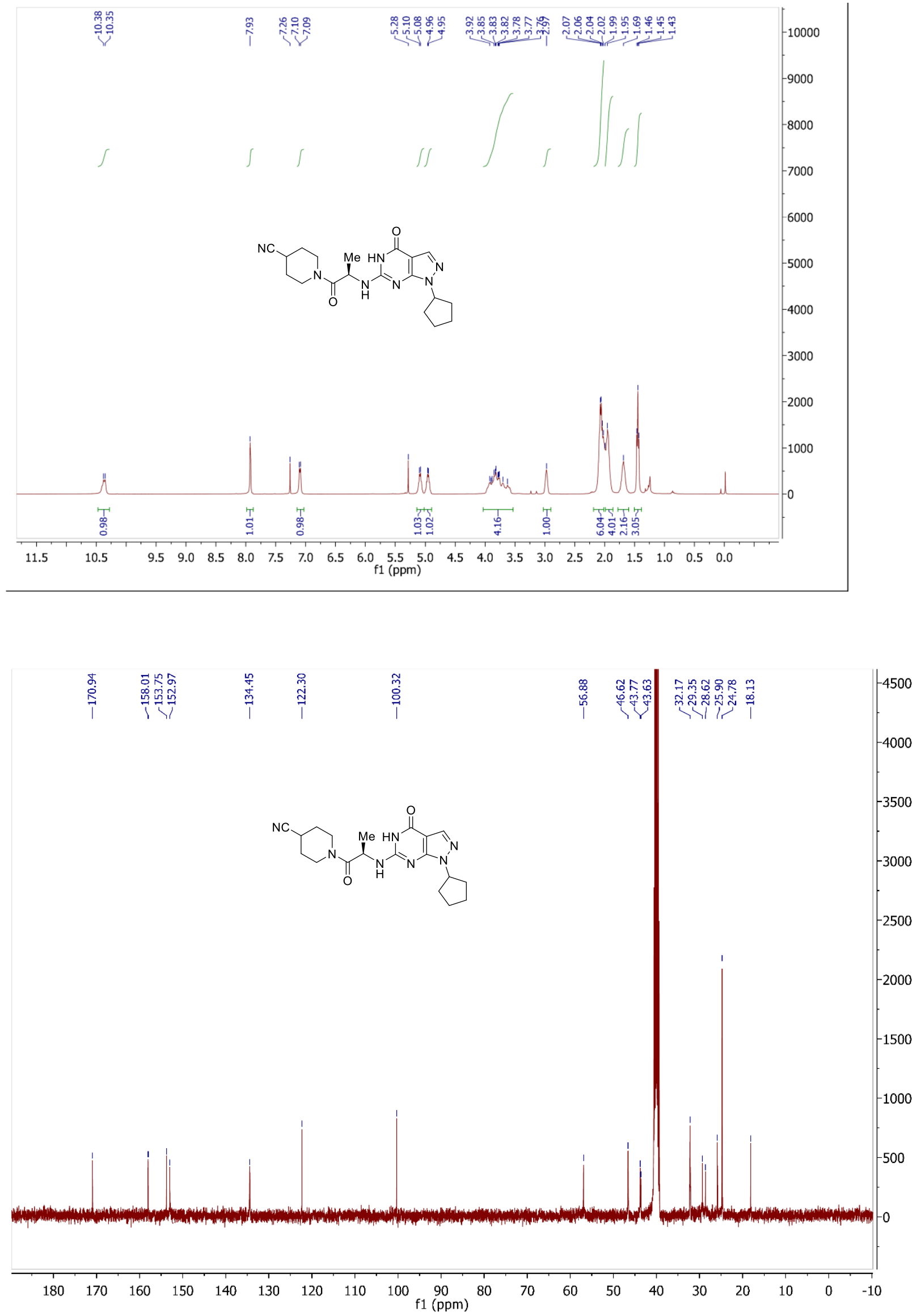


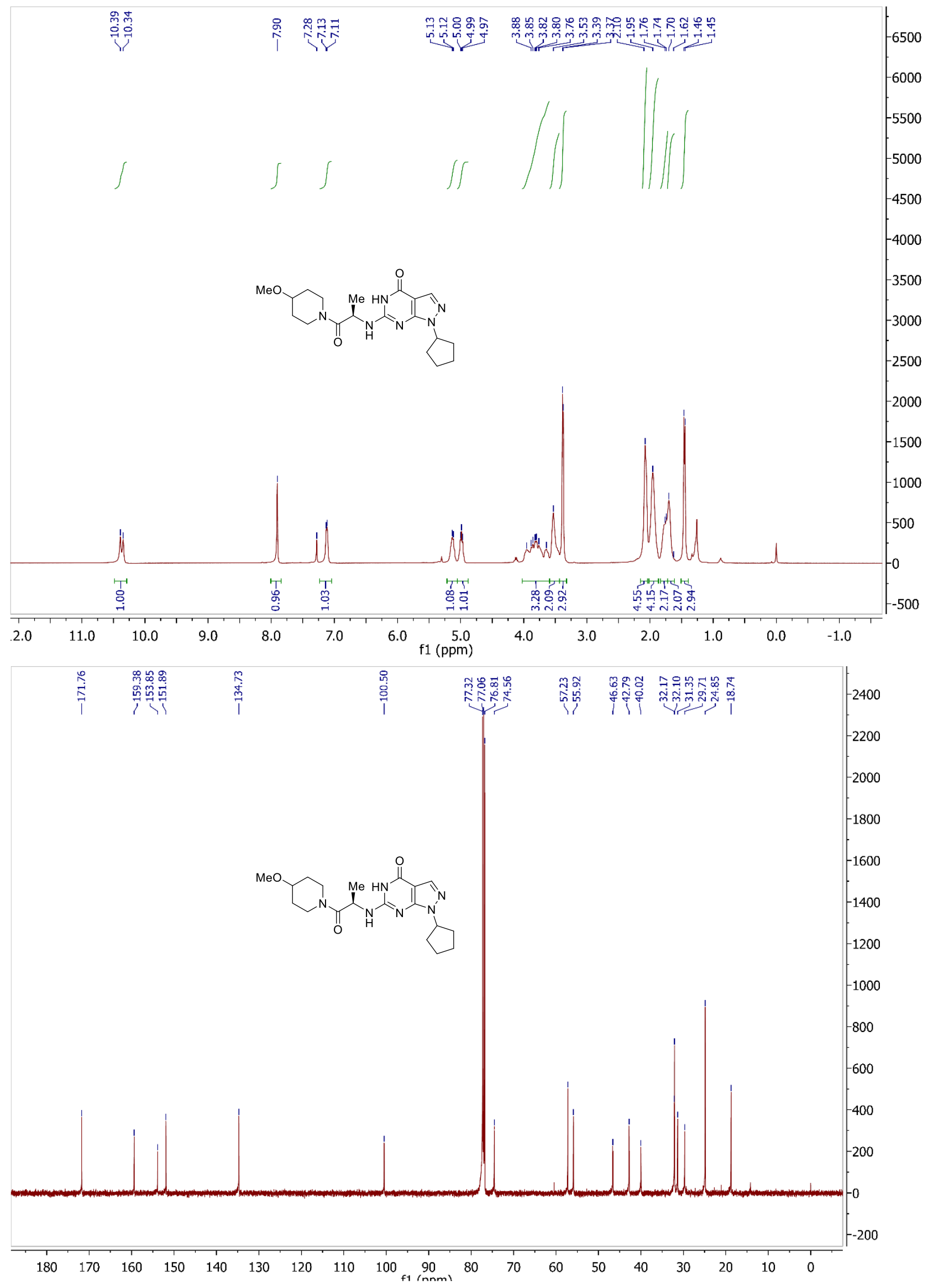




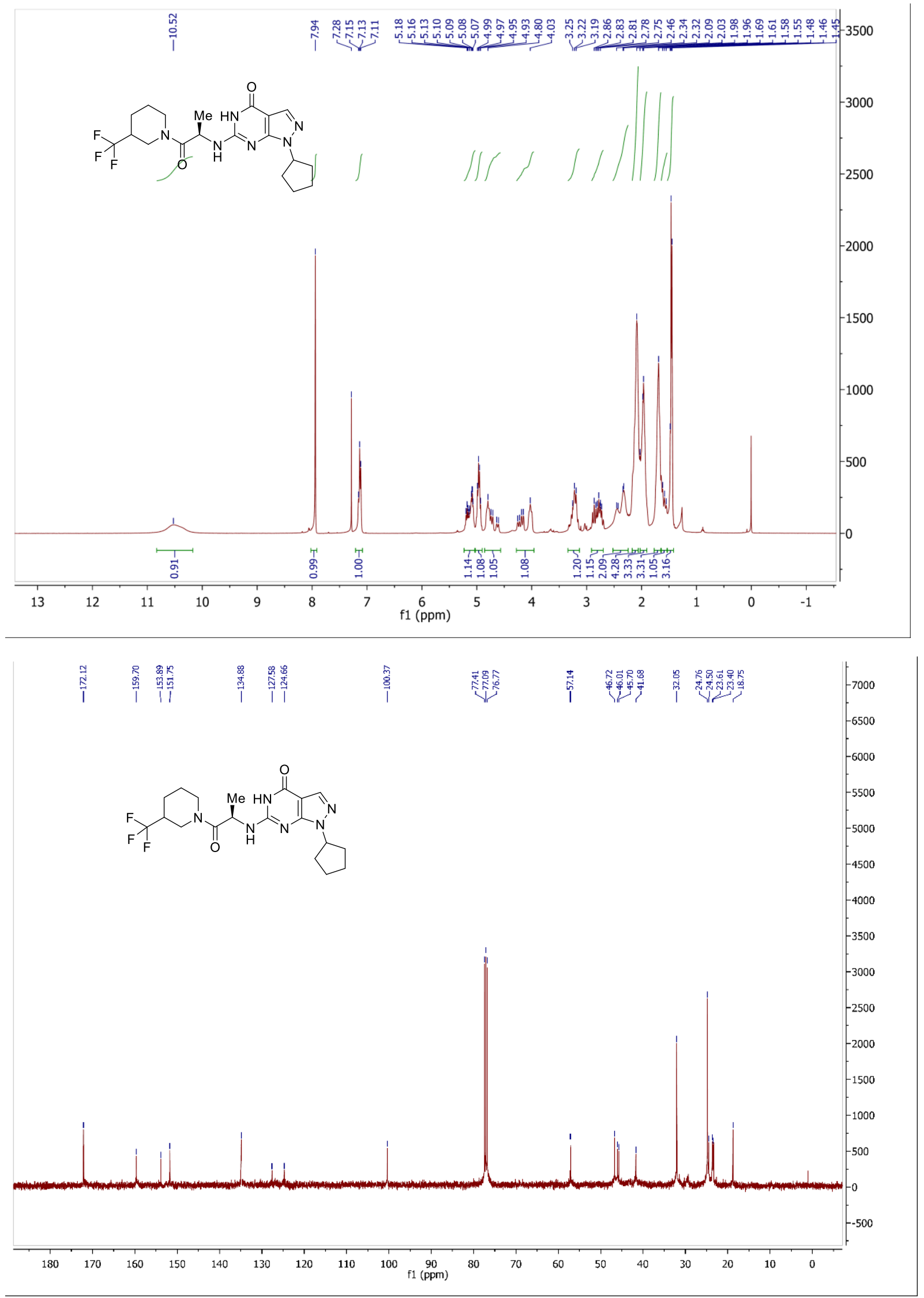




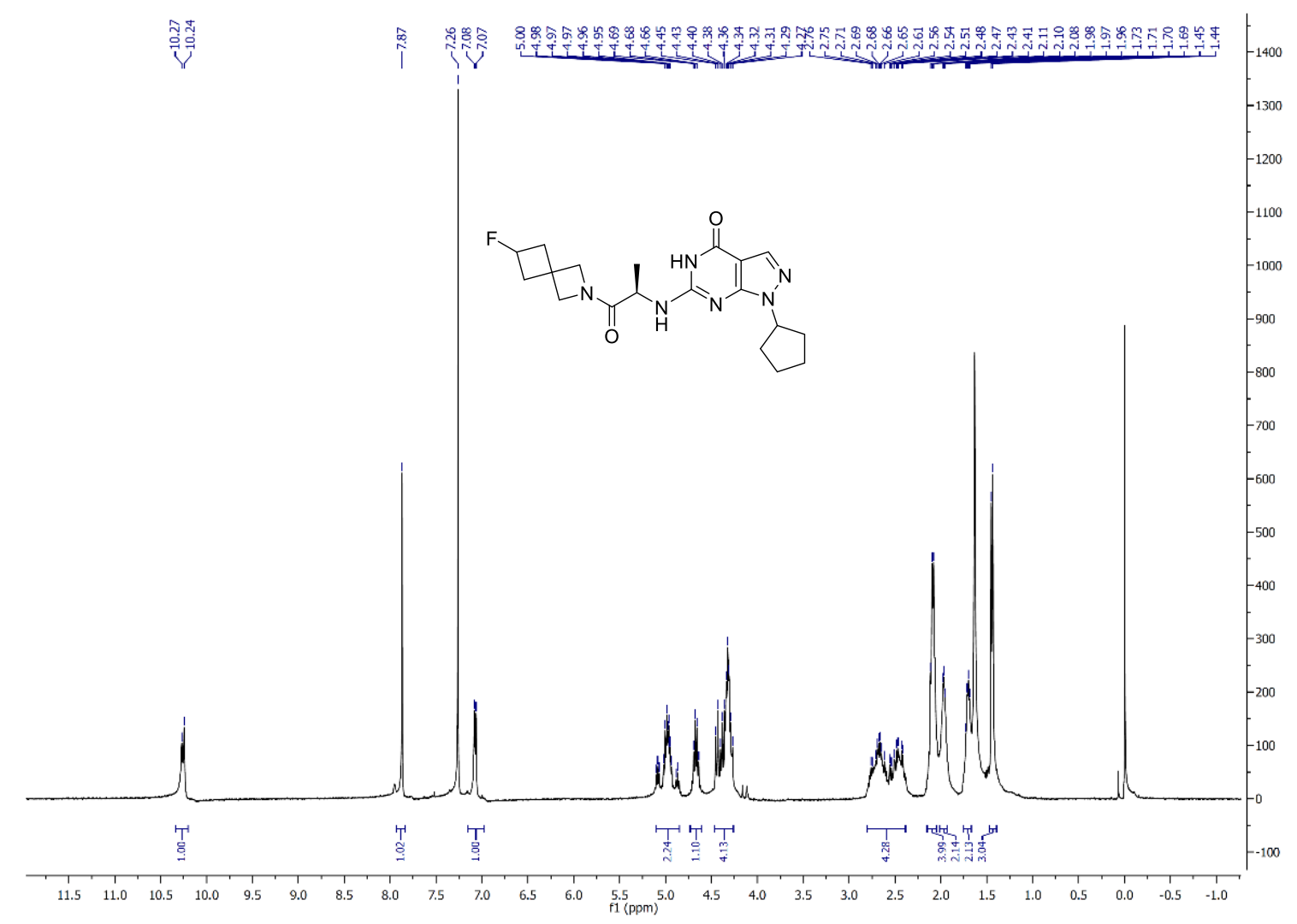

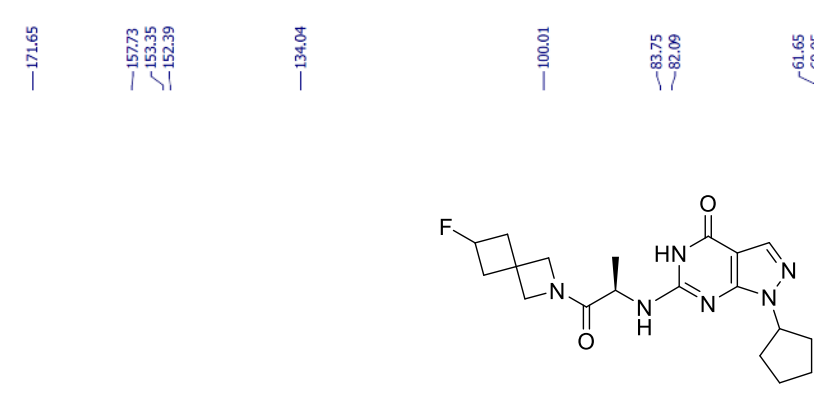

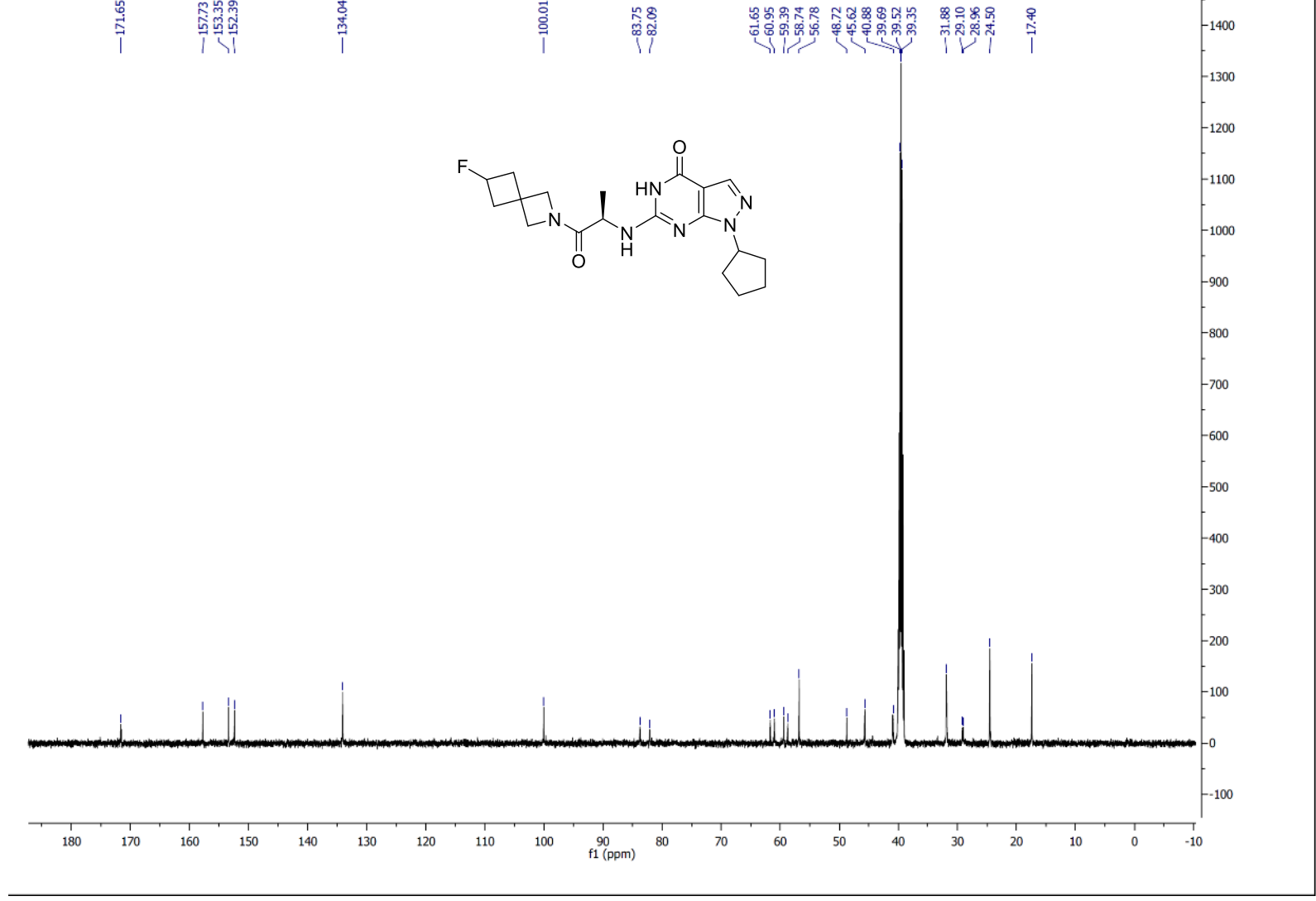




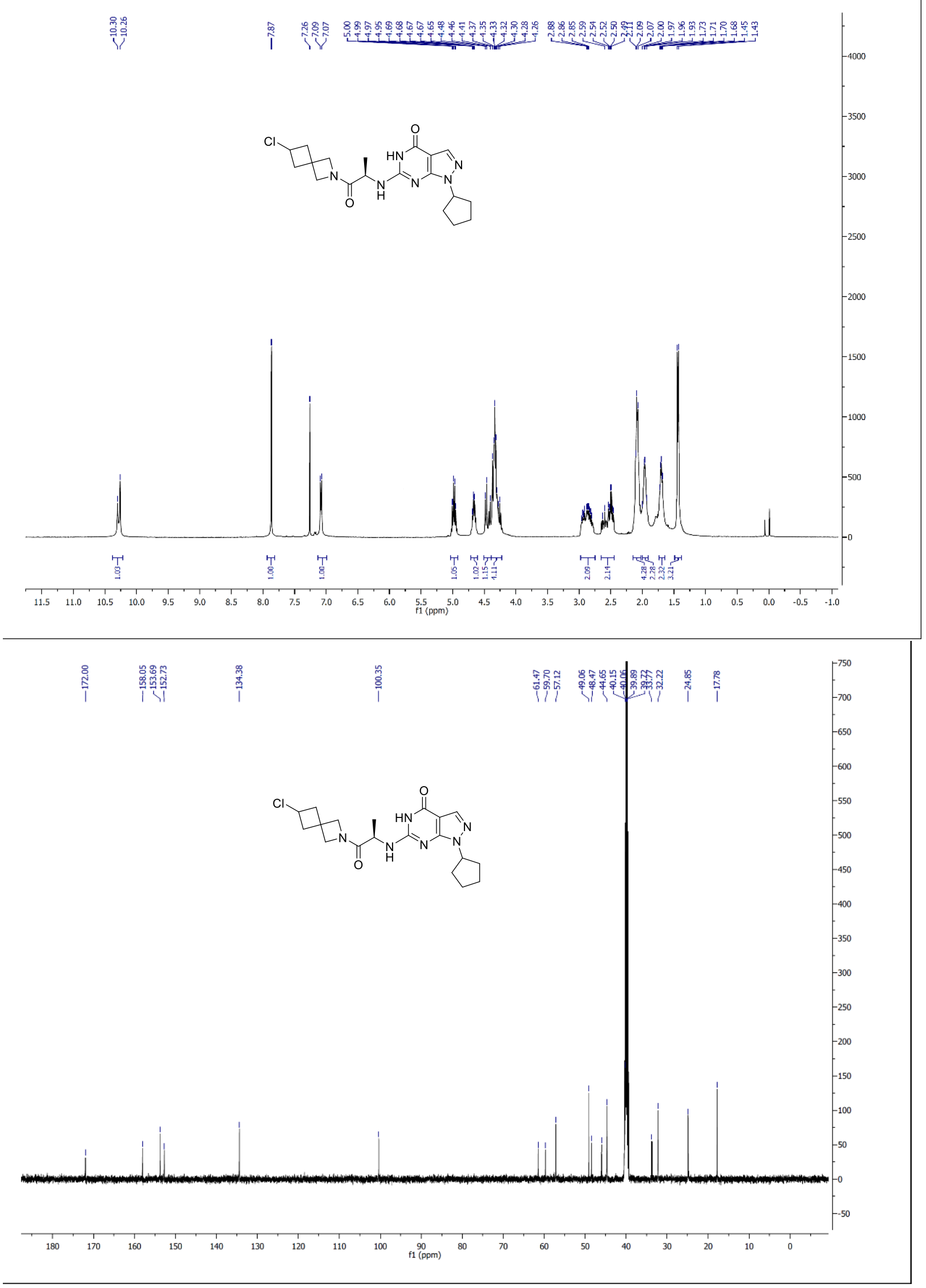




\section{HRMS spectrum.}

\begin{tabular}{|c|c|c|c|c|c|c|c|c|c|c|c|}
\hline Elmt & Val. & Min & Max & Elmt & Val. & Min & Max & Elmt & Val. & Min & Max \\
\hline $\mathrm{H}$ & 1 & 12 & 38 & $\mathrm{~N}$ & 3 & 0 & 8 & $P$ & 3 & 0 & 0 \\
\hline $2 \mathrm{H}$ & 1 & 0 & 0 & 0 & 2 & 1 & 5 & $S$ & 2 & 0 & 0 \\
\hline B & 3 & 0 & 0 & $F$ & 1 & 0 & 0 & $\mathrm{Cl}$ & 1 & 0 & 2 \\
\hline C & 4 & 12 & 20 & $\mathrm{Si}$ & 4 & 0 & 0 & $\mathrm{Se}$ & 2 & 0 & 0 \\
\hline
\end{tabular}

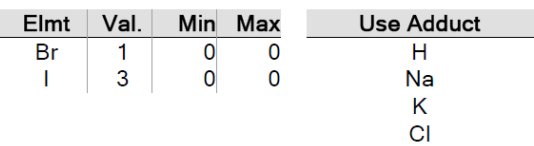
Error Margin (ppm): 30 HC Ratio: unlimited
Max Isotopes: all

DBE Range: -1.0 - 1000.0

MSn Iso RI (\%): 75.00

Max Results: 40

Event\#: $1 \mathrm{MS}(\mathrm{E}+)$ Ret. Time : 0.987 Scan\# : 198

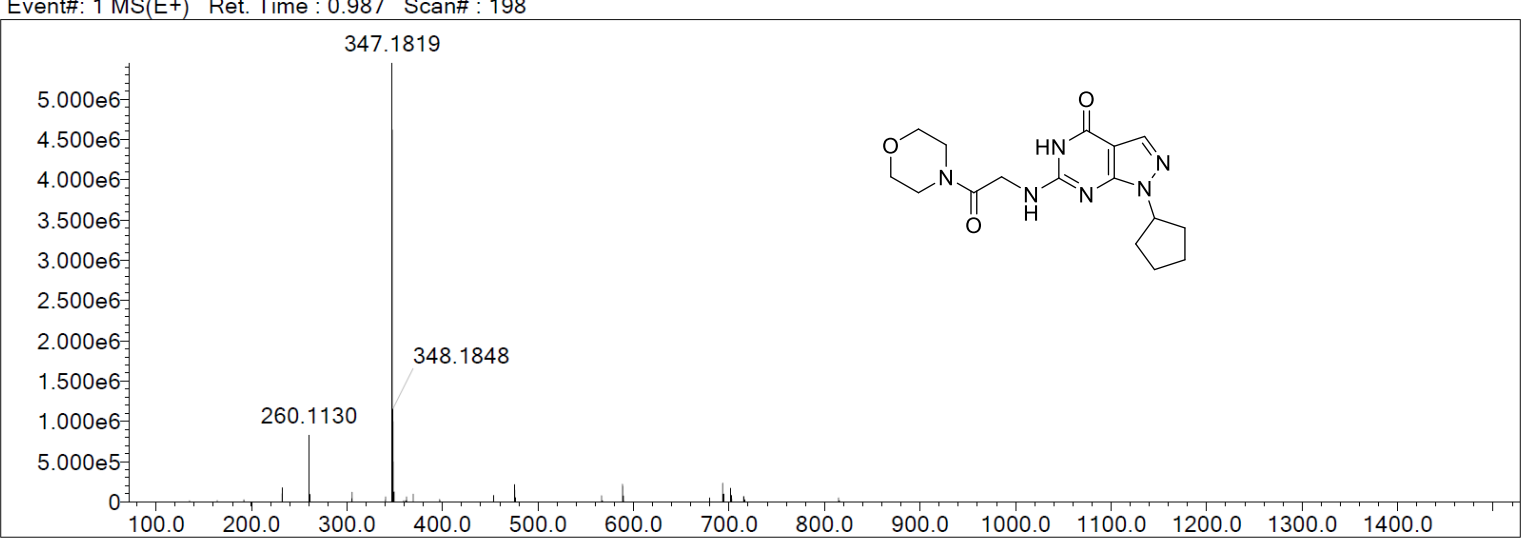

Measured region for $347.1819 \mathrm{~m} / \mathrm{z}$

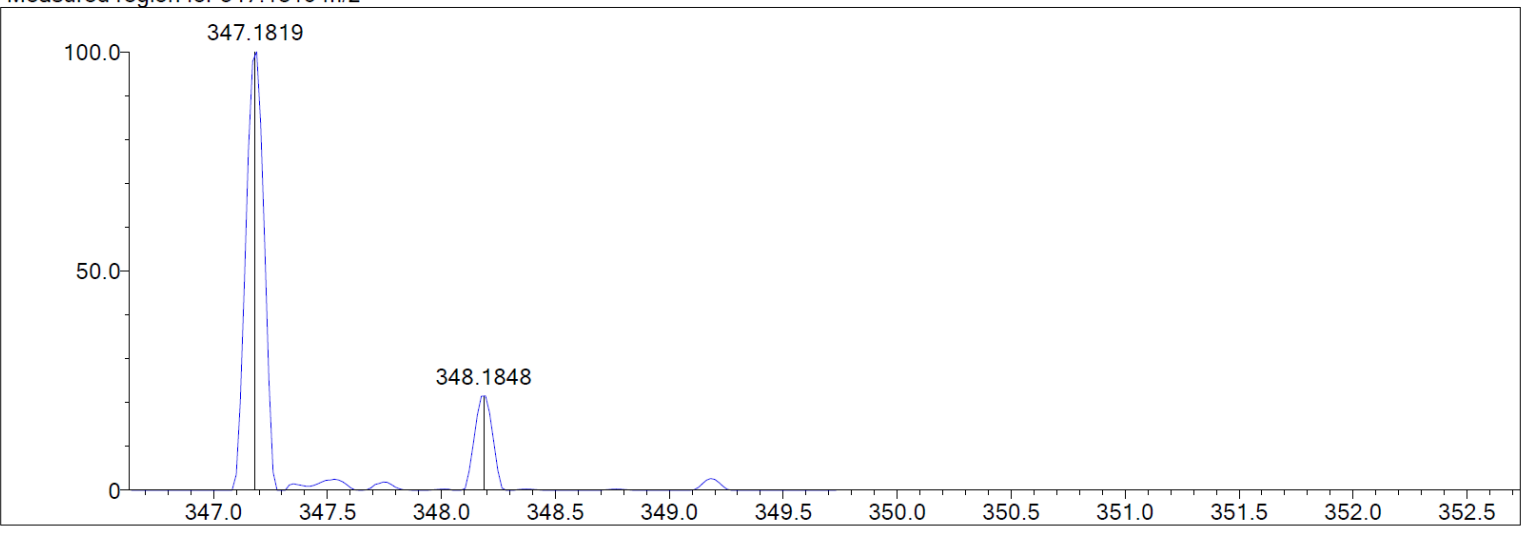

C16 H22 N6 O3 [M+H]+ : Predicted region for $347.1826 \mathrm{~m} / \mathrm{z}$

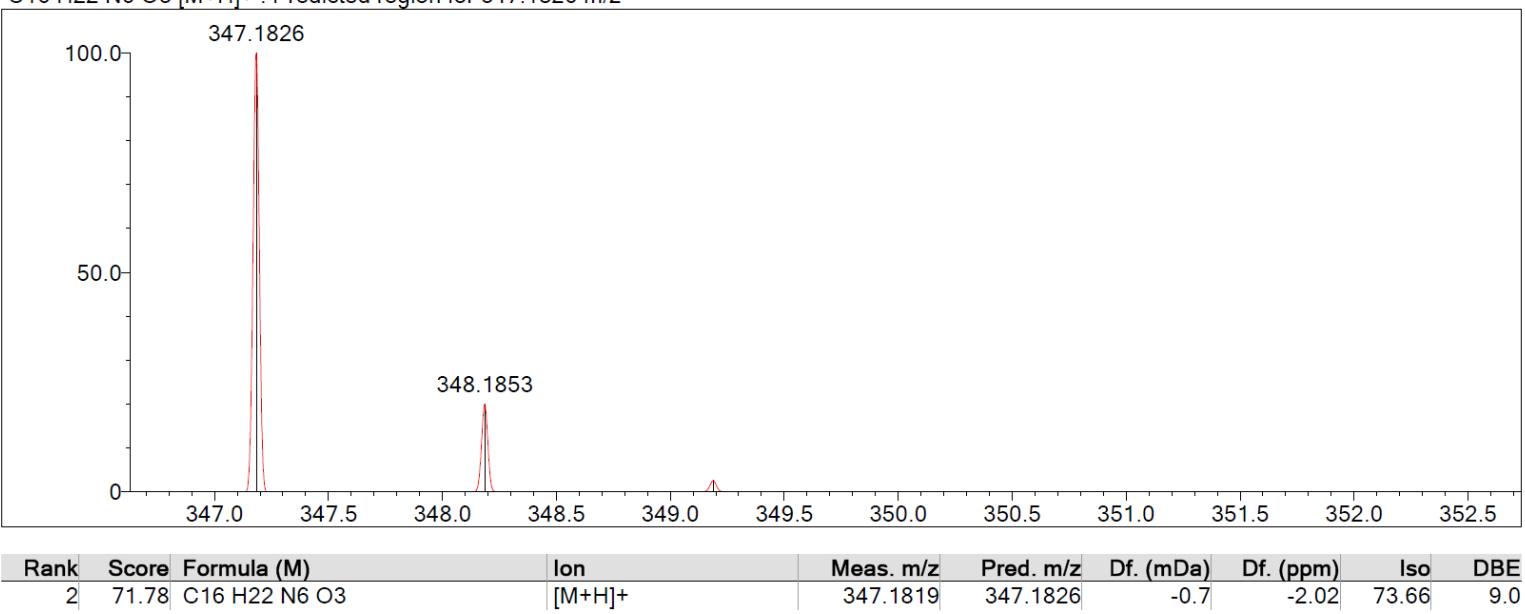




\begin{tabular}{c|r|r|r} 
Elmt & Val. & Min & Max \\
\hline $\mathrm{H}$ & 1 & 12 & 38 \\
$2 \mathrm{H}$ & 1 & 0 & 0 \\
$\mathrm{~B}$ & 3 & 0 & 0 \\
$\mathrm{C}$ & 4 & 12 & 20 \\
$\mathrm{~N}$ & 3 & 0 & 8 \\
$\mathrm{O}$ & 2 & 1 & 5
\end{tabular}

Error Margin (ppm): 30 HC Ratio: unlimited Max Isotopes: all MSn Iso RI (\%): 75.00

\begin{tabular}{c|r|r|r} 
Elmt & Val. & Min & Max \\
\hline F & 1 & 0 & 4 \\
Si & 4 & 0 & 0 \\
$\mathrm{P}$ & 3 & 0 & 0 \\
$\mathrm{~S}$ & 2 & 0 & 2 \\
$\mathrm{Cl}$ & 1 & 0 & 2 \\
$\mathrm{Se}$ & 2 & 0 & 0
\end{tabular}

DBE Range: -1.0 - 1000.0

Apply N Rule: no

Isotope RI (\%): 1.00

MSn Logic Mode: AND

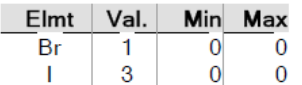

Use Adduct
$\mathrm{H}$
$\mathrm{Na}$
$\mathrm{K}$
$\mathrm{NH} 4$
$\mathrm{~F}$
$\mathrm{Cl}$

Electron lons: both Use MSn Info: no

Isotope Res: 10000

Max Results: 40

Event\#: $1 \mathrm{MS}\left(\mathrm{E}^{+}\right)$Ret. Time : 0.907 Scan\# : 182

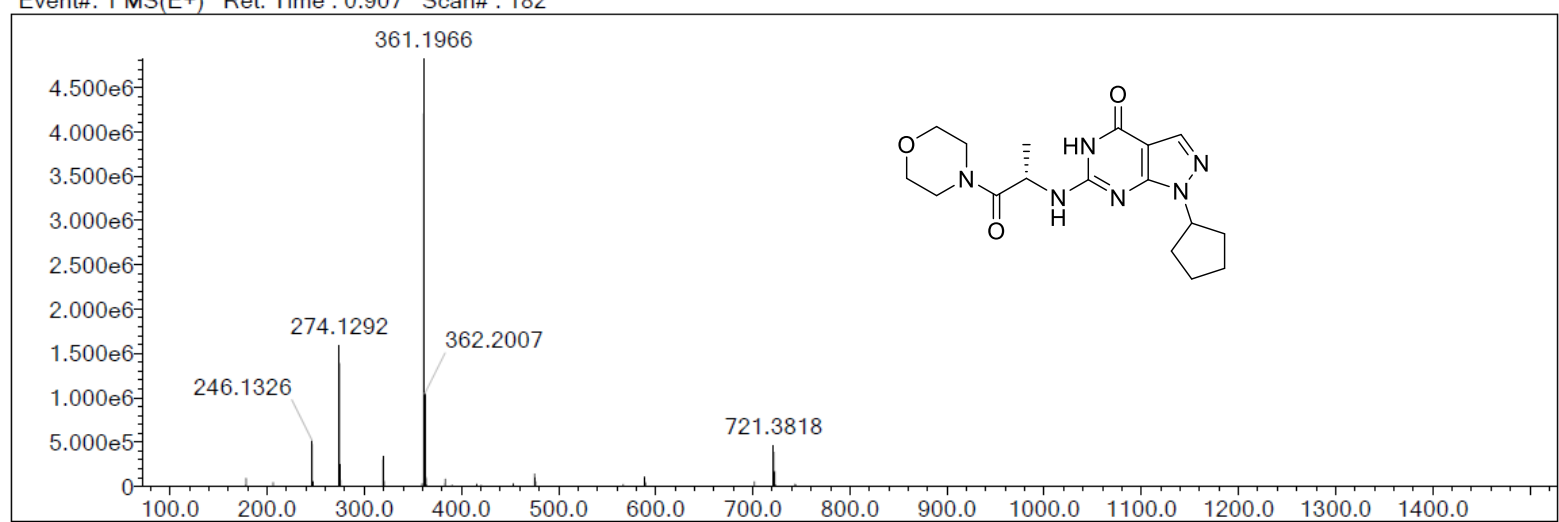

Measured region for $361.1966 \mathrm{~m} / \mathrm{z}$

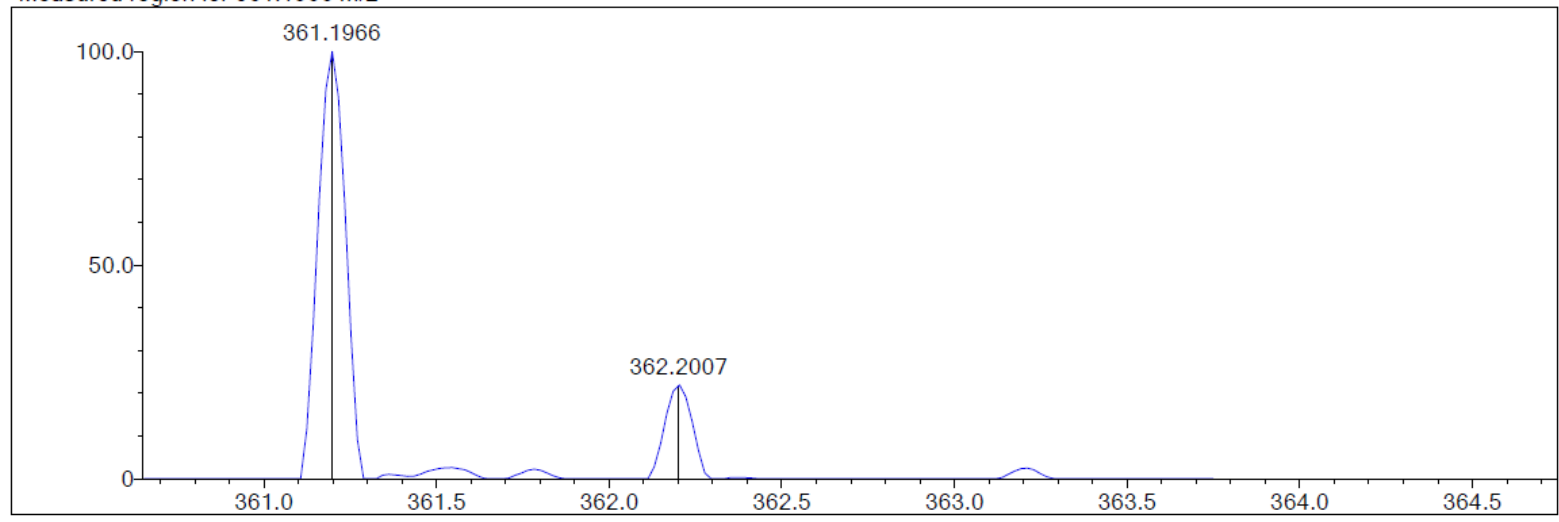

C17 H24 N6 O3 [M+H]+ : Predicted region for $361.1983 \mathrm{~m} / \mathrm{z}$

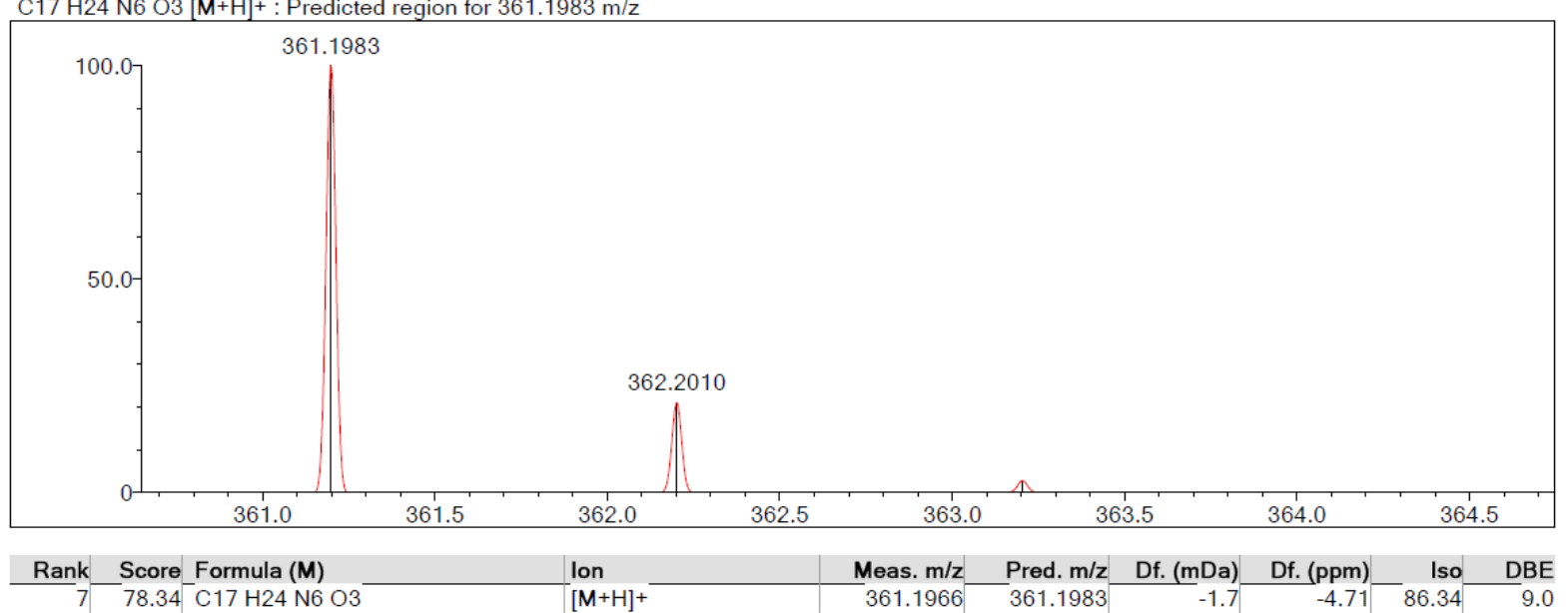




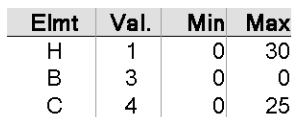

Error Margin (ppm): 20 HC Ratio: unlimited Max Isotopes: all

MSn Iso RI (\%): 75.00

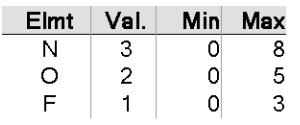

\begin{tabular}{c|c|r|r} 
Elmt & Val. & Min & Max \\
\hline $\mathrm{P}$ & 3 & 0 & 0 \\
$\mathrm{~S}$ & 2 & 0 & 1 \\
$\mathrm{Cl}$ & 1 & 0 & 3
\end{tabular}

DBE Range: $-1.0-1000.0$ Apply N Rule: no

Isotope RI (\%): 1.00

MSn Logic Mode: AND \begin{tabular}{c|c|rr} 
Elmt & Val. & Min & Max \\
\hline $\mathrm{Se}$ & 2 & 0 & 0 \\
$\mathrm{Br}$ & 1 & 0 & 0 \\
$\mathrm{I}$ & 3 & 0 & 0
\end{tabular}

Use Adduct

Electron lons: both

Use MSn Info: no

Isotope Res: 10000

Max Results: 40

Event\#: $1 \mathrm{MS}(\mathrm{E}+)$ Ret. Time : 0.937 Scan\# : 188

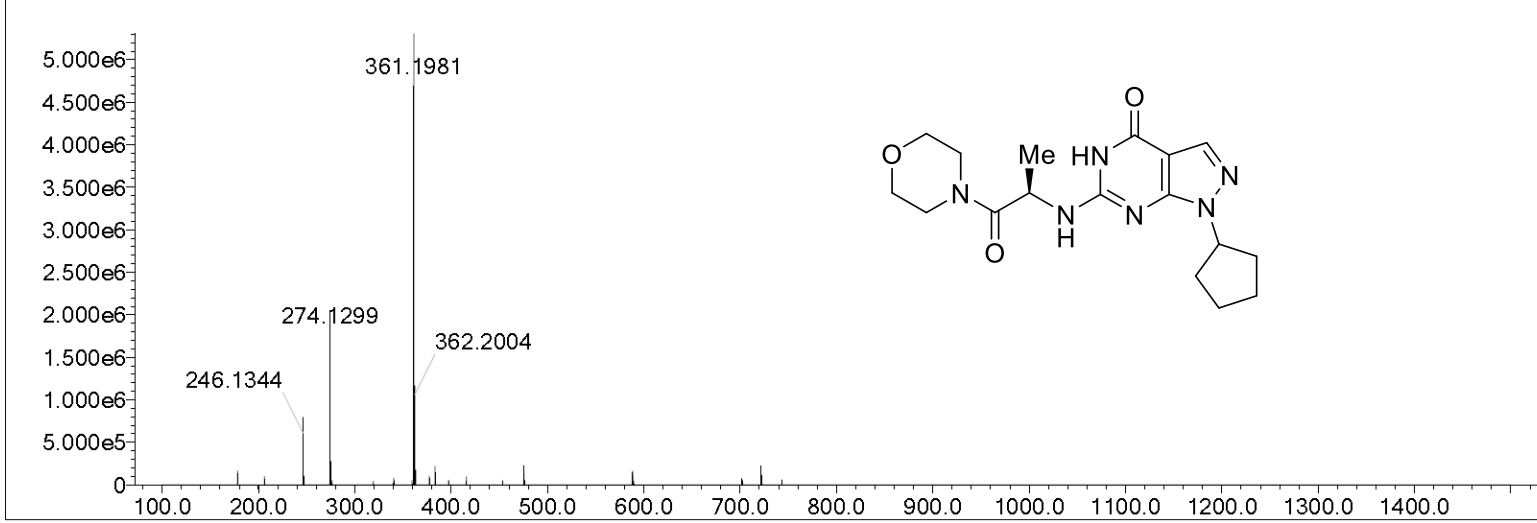

Measured region for $361.1981 \mathrm{~m} / \mathrm{z}$

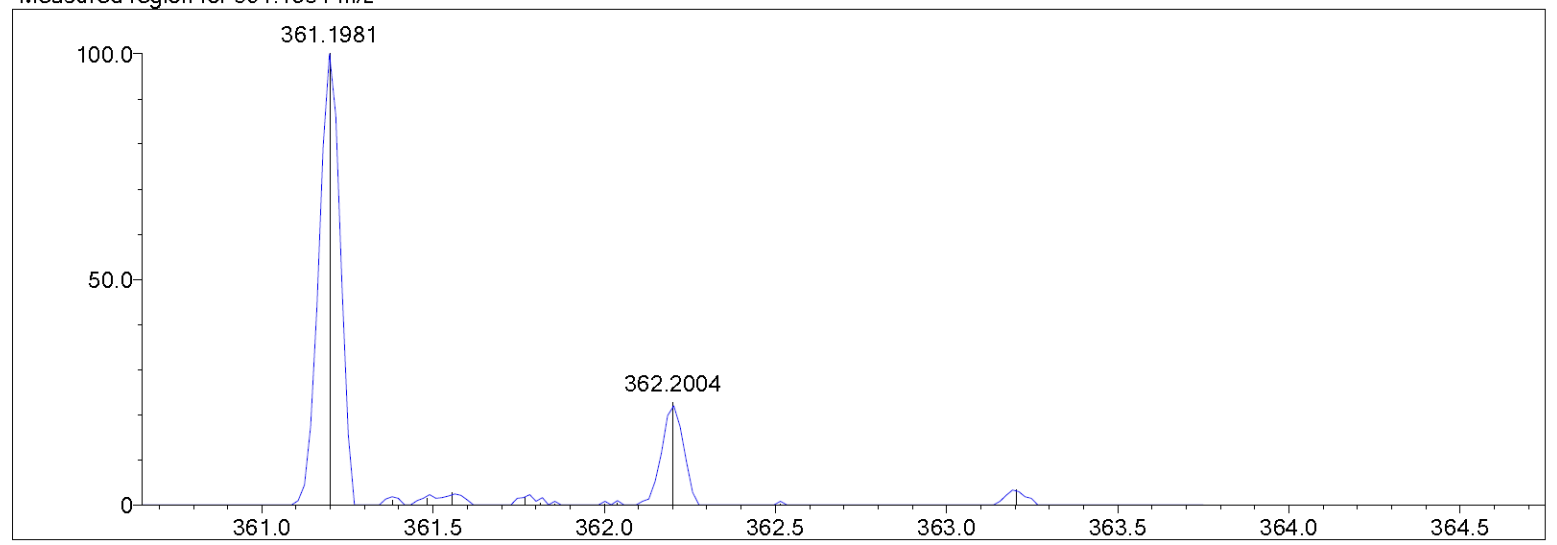

C17 H24 N6 O3 $[\mathrm{M}+\mathrm{H}]+$ : : Predicted region for $361.1983 \mathrm{~m} / \mathrm{z}$

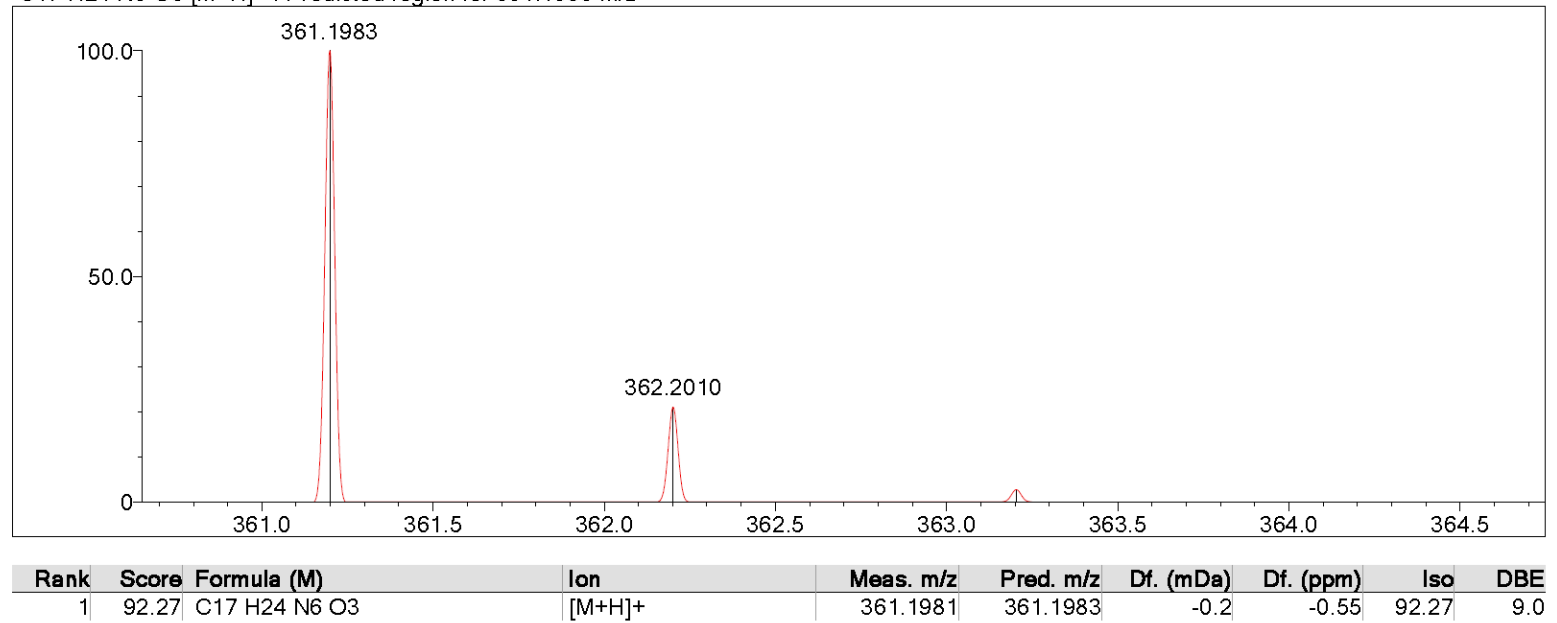




\begin{tabular}{c|c|r|r} 
Elmt & Val. & Min & Max \\
\hline $\mathrm{H}$ & 1 & 12 & 38 \\
$2 \mathrm{H}$ & 1 & 0 & 0 \\
$\mathrm{~B}$ & 3 & 0 & 0 \\
$\mathrm{C}$ & 4 & 12 & 20 \\
$\mathrm{~N}$ & 3 & 0 & 8 \\
$\mathrm{O}$ & 2 & 1 & 5
\end{tabular}

Error Margin (ppm): 30 HC Ratio: unlimited Max Isotopes: all MSn Iso RI (\%): 75.00

\begin{tabular}{c|c|rr} 
Elmt & Val. & Min & Max \\
\hline $\mathrm{F}$ & 1 & 0 & 4 \\
$\mathrm{Si}$ & 4 & 0 & 0 \\
$\mathrm{P}$ & 3 & 0 & 0 \\
$\mathrm{~S}$ & 2 & 0 & 2 \\
$\mathrm{Cl}$ & 1 & 0 & 2 \\
$\mathrm{Se}$ & 2 & 0 & 0
\end{tabular}

DBE Range: -1.0 - 1000.0 Apply N Rule: no Isotope RI (\%): 1.00 MSn Logic Mode: AND

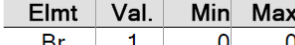

\begin{tabular}{c|c|r|r}
\hline $\mathrm{Br}$ & 1 & 0 & 0 \\
$\mathrm{I}$ & 3 & 0 & 0
\end{tabular}

\begin{tabular}{c} 
Use Adduct \\
\hline $\mathrm{H}$ \\
$\mathrm{Na}$ \\
$\mathrm{K}$ \\
$\mathrm{NH} 4$ \\
$\mathrm{~F}$ \\
$\mathrm{Cl}$
\end{tabular}

Electron lons: both Use MSn Info: no Isotope Res: 10000 Max Results: 40

Event\#: $1 \mathrm{MS}\left(\mathrm{E}^{+}\right)$Ret. Time : 0.907 Scan\# : 182

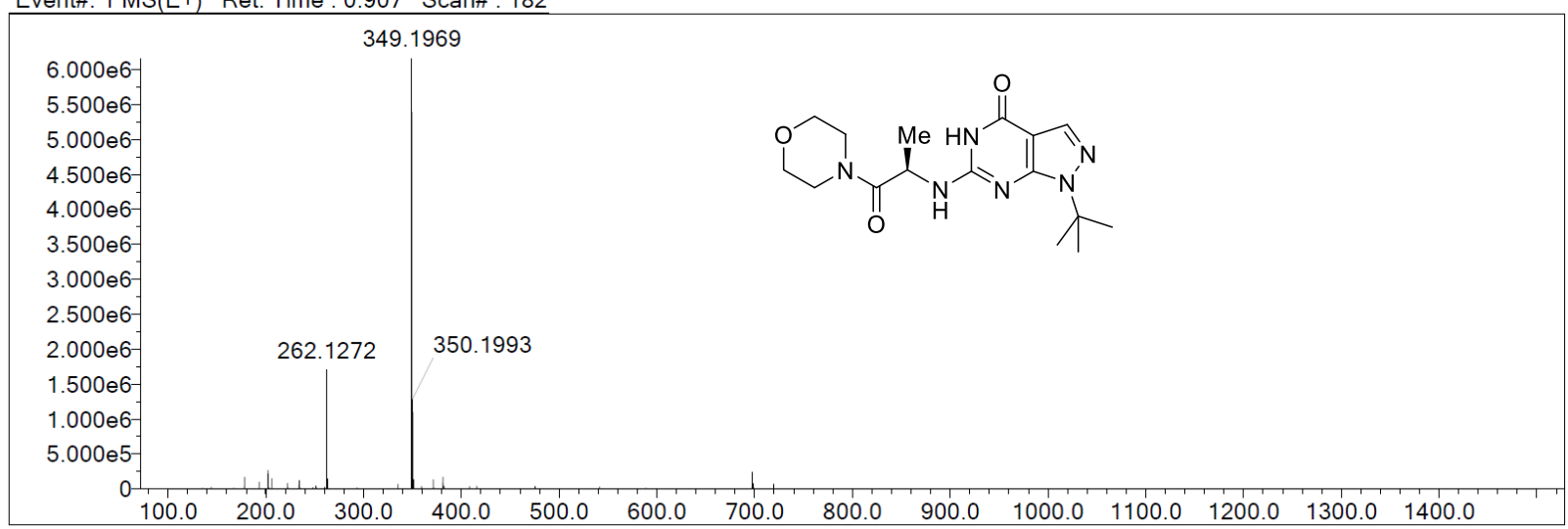

Measured region for $349.1969 \mathrm{~m} / \mathrm{z}$

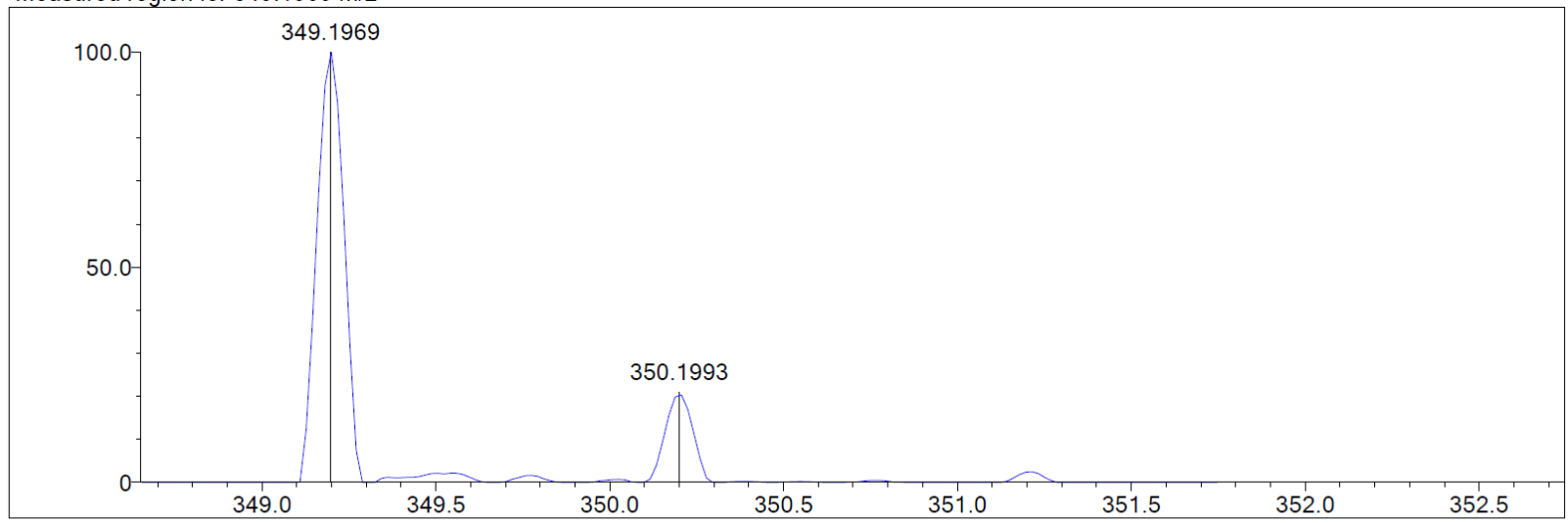

C16 H24 N6 O3 [M+H]+ : Predicted region for $349.1983 \mathrm{~m} / \mathrm{z}$

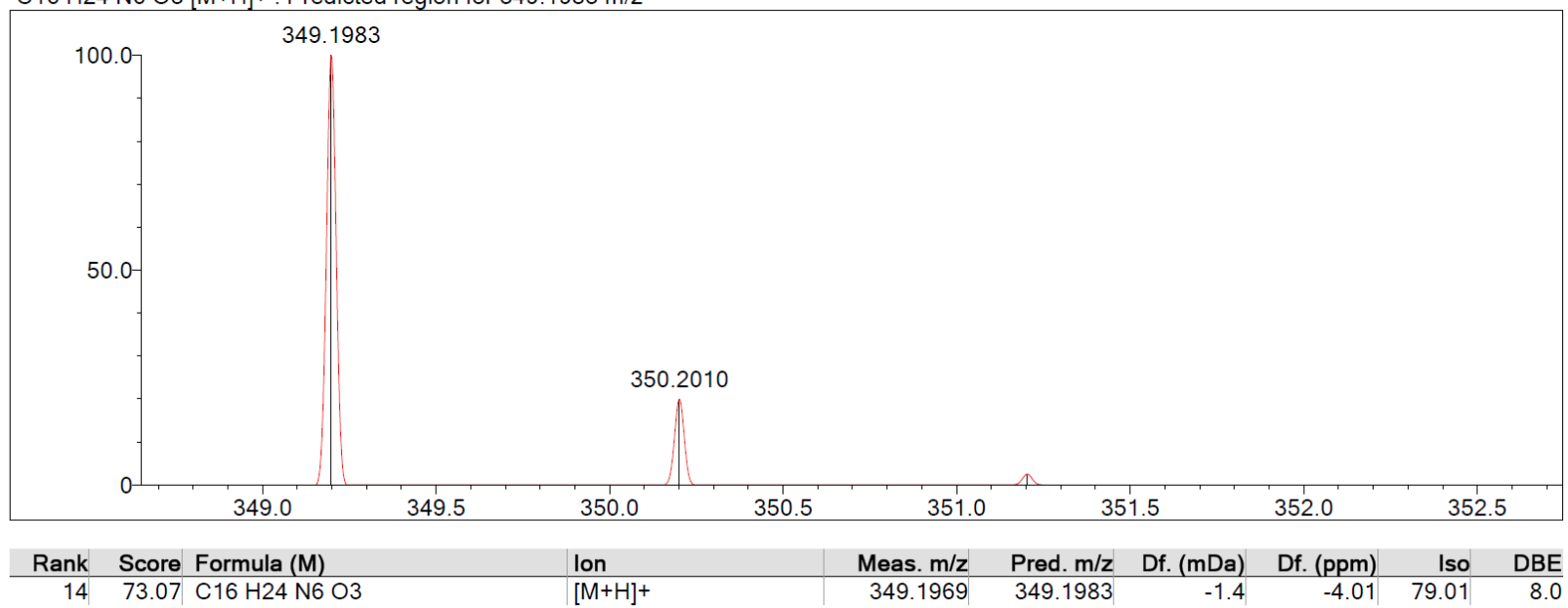




\begin{tabular}{c|c|r|r} 
Elmt & Val. & Min & Max \\
\hline $\mathrm{H}$ & 1 & 0 & 30 \\
$\mathrm{~B}$ & 3 & 0 & 0 \\
$\mathrm{C}$ & 4 & 0 & 25
\end{tabular}

Error Margin (ppm): 20 HC Ratio: unlimited Max Isotopes: all MSn Iso RI (\%): 75.00

\section{\begin{tabular}{c|c|c|c} 
Elmt & Val. Min Max \\
\hline & & 3
\end{tabular}

\begin{tabular}{|c|c|c|}
\hline $\mathrm{N}$ & 3 & 0 \\
\hline 0 & 2 & 0 \\
\hline$F$ & 1 & 0 \\
\hline
\end{tabular}

DBE Range: -1.0 - 1000.0

Apply N Rule: no

Isotope RI (\%): 1.00

MSn Logic Mode: AND

\begin{tabular}{c|c|rr} 
Elmt & Val. & Min & Max \\
\hline $\mathrm{P}$ & 3 & 0 & 0 \\
$\mathrm{~S}$ & 2 & 0 & 1 \\
$\mathrm{Cl}$ & 1 & 0 & 3
\end{tabular}

Elmt Val. Min Max

\begin{tabular}{c|c|ccc}
$\mathrm{Se}$ & 2 & 0 & 0 & $\mathrm{H}$ \\
$\mathrm{Br}$ & 1 & 0 & 0 & $\mathrm{Na}$ \\
\hline & 3 & 0 & 0 &
\end{tabular}

\begin{tabular}{c|c|ccc}
$\mathrm{Se}$ & 2 & 0 & 0 & $\mathrm{H}$ \\
$\mathrm{Br}$ & 1 & 0 & 0 & $\mathrm{Na}$ \\
\hline & 3 & 0 & 0 &
\end{tabular}

Electron lons: both

Use MSn Info: no

Isotope Res: 10000

Max Results: 40

Event\#: $1 \mathrm{MS}(\mathrm{E}+)$ Ret. Time : 0.887 Scan\# : 178

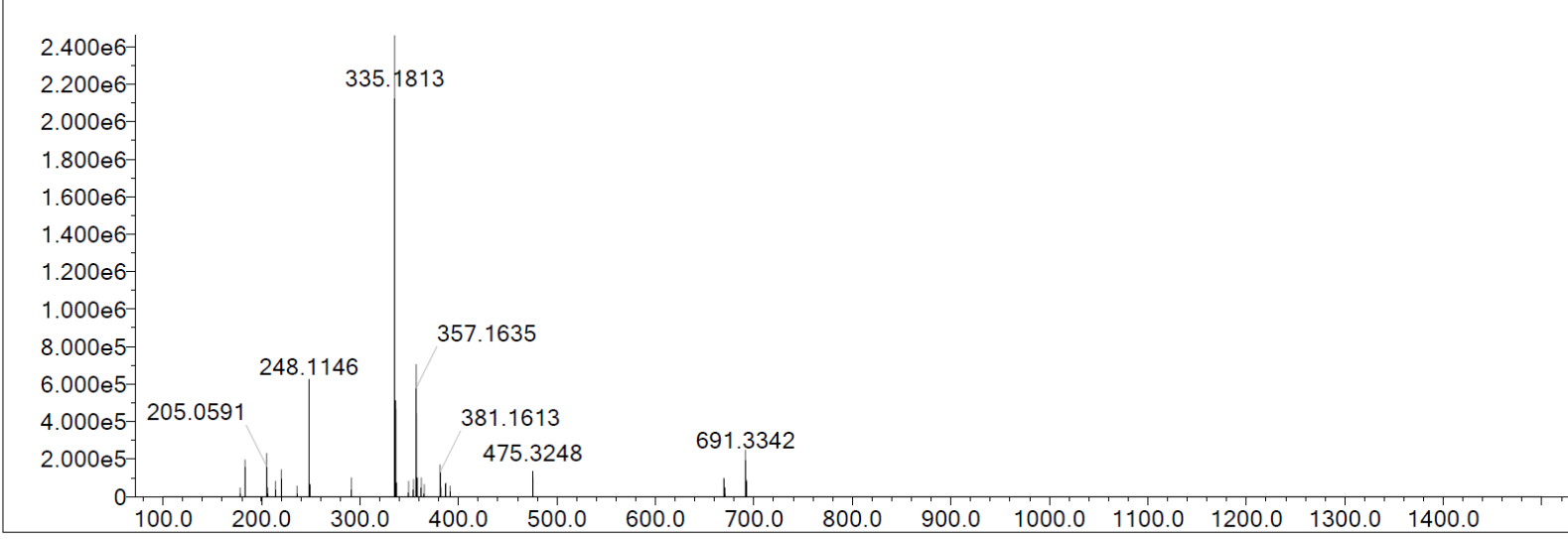

Measured region for $335.1813 \mathrm{~m} / \mathrm{z}$

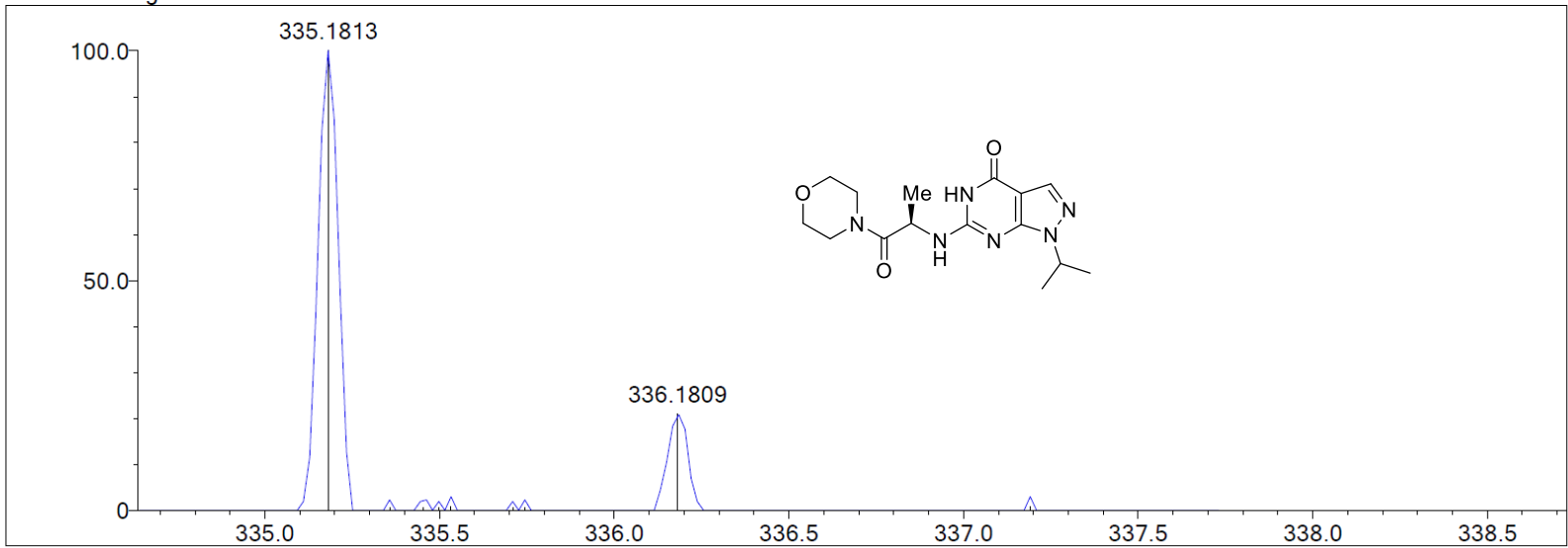

$\mathrm{C} 15 \mathrm{H} 22 \mathrm{~N} 6 \mathrm{O} 3[\mathrm{M}+\mathrm{H}]+$ : Predicted region for $335.1826 \mathrm{~m} / \mathrm{z}$

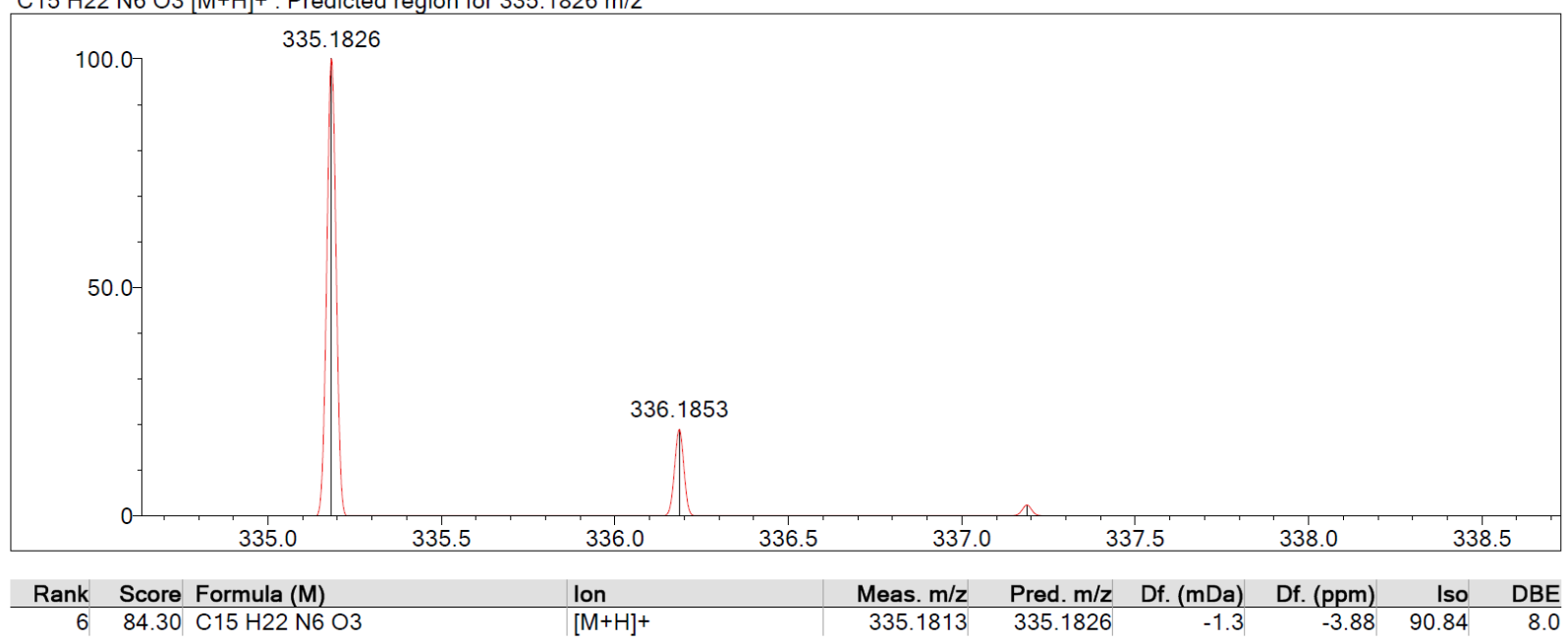




\begin{tabular}{c|r|rrr|r|rrr|r|rr} 
Elmt & Val. & Min & Max & Elmt & Val. & Min & Max & Elmt & Val. & Min & Max \\
\hline $\mathrm{H}$ & 1 & 0 & 30 & $\mathrm{~N}$ & 3 & 0 & 8 & $\mathrm{P}$ & 3 & 0 & 0 \\
$\mathrm{~B}$ & 3 & 0 & 0 & $\mathrm{O}$ & 2 & 0 & 5 & $\mathrm{~S}$ & 2 & 0 & 1 \\
$\mathrm{C}$ & 4 & 0 & 25 & $\mathrm{~F}$ & 1 & 0 & 3 & $\mathrm{Cl}$ & 1 & 0 & 3
\end{tabular}

Elmt Val. Min Max

Use Adduct

\begin{tabular}{l|l|l|ll}
$\mathrm{Se}$ & 2 & 0 & 0 & $\mathrm{H}$ \\
$\mathrm{Br}$ & 1 & 0 & 0 &
\end{tabular}

$\mathrm{H}$
$\mathrm{Na}$

Error Margin (ppm): 20 HC Ratio: unlimited

Max Isotopes: all

DBE Range: $-1.0-1000.0$

Apply N Rule: no

Isotope RI (\%): 1.00

\begin{tabular}{l|l|l|l}
1 & 3 & 0 & 0
\end{tabular}

Na

MSn Iso RI (\%): 75.00

MSn Logic Mode: AND

Electron lons: both

Use MSn Info: no

Isotope Res: 10000

Max Results: 40

Event\#: $1 \mathrm{MS}(\mathrm{E}+)$ Ret. Time : 0.887 Scan\# : 178

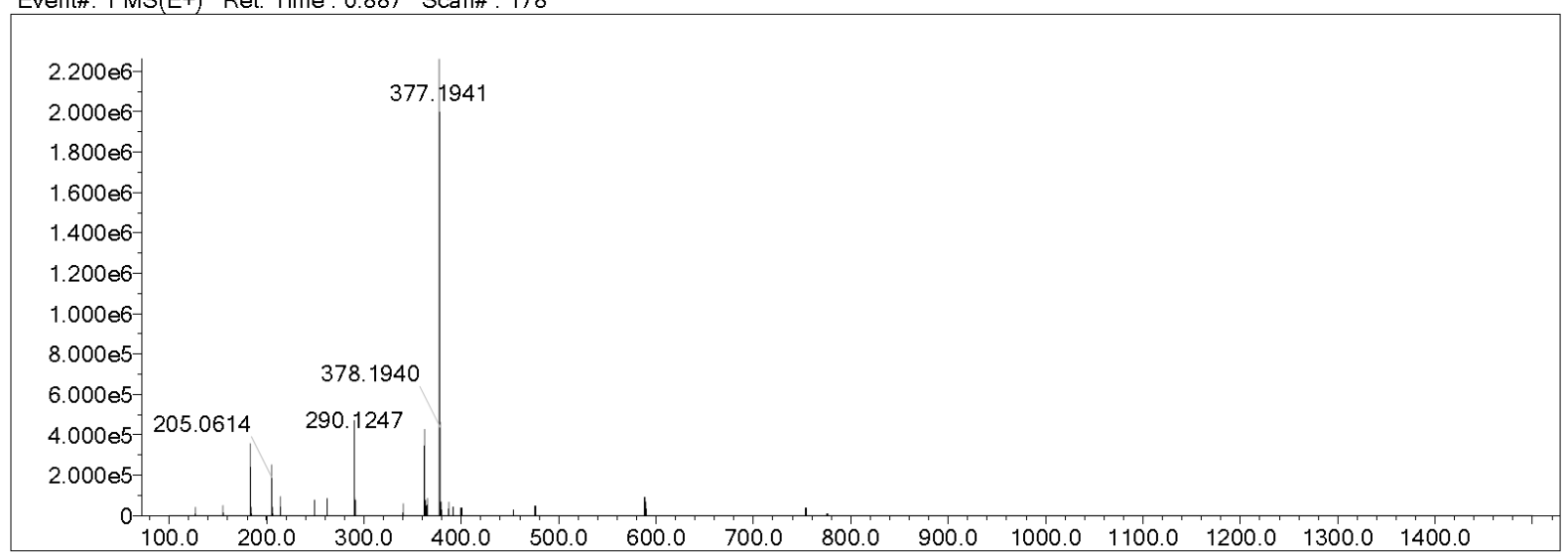

Measured region for $377.1941 \mathrm{~m} / \mathrm{z}$

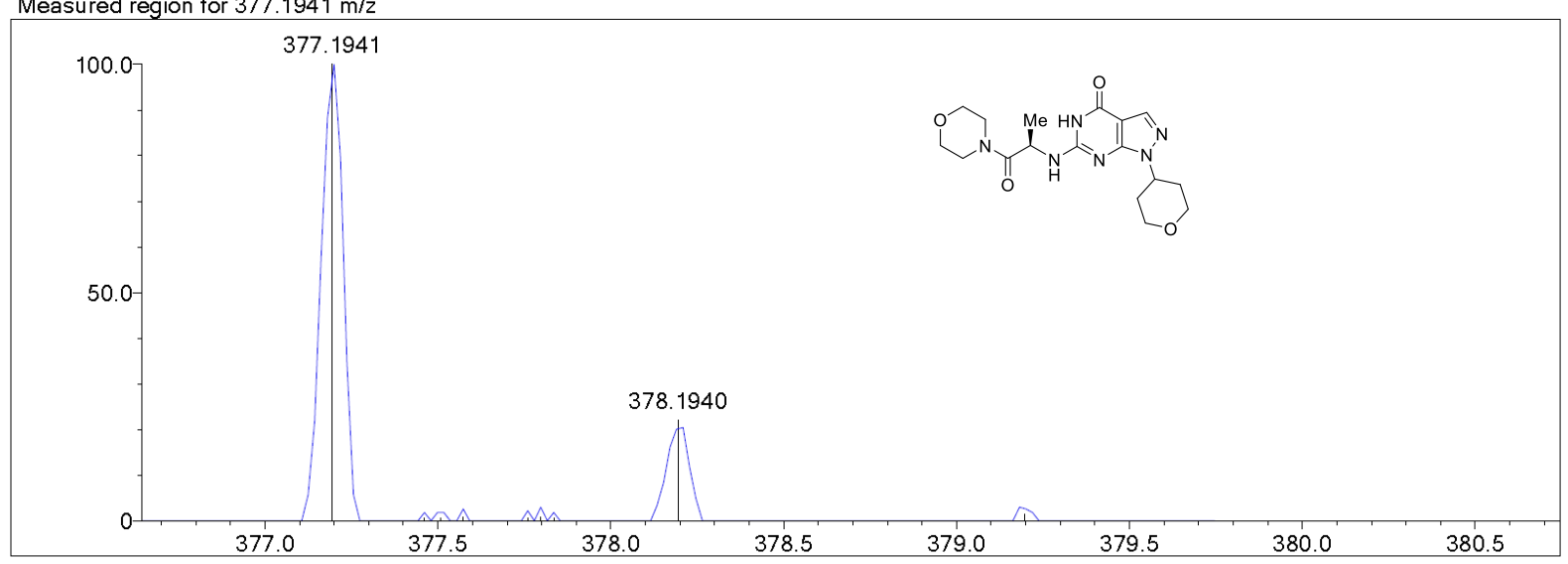

$\mathrm{C} 17 \mathrm{H} 24 \mathrm{~N} 6 \mathrm{O} 4[\mathrm{M}+\mathrm{H}]+$ : : Predicted region for $377.1932 \mathrm{~m} / \mathrm{z}$

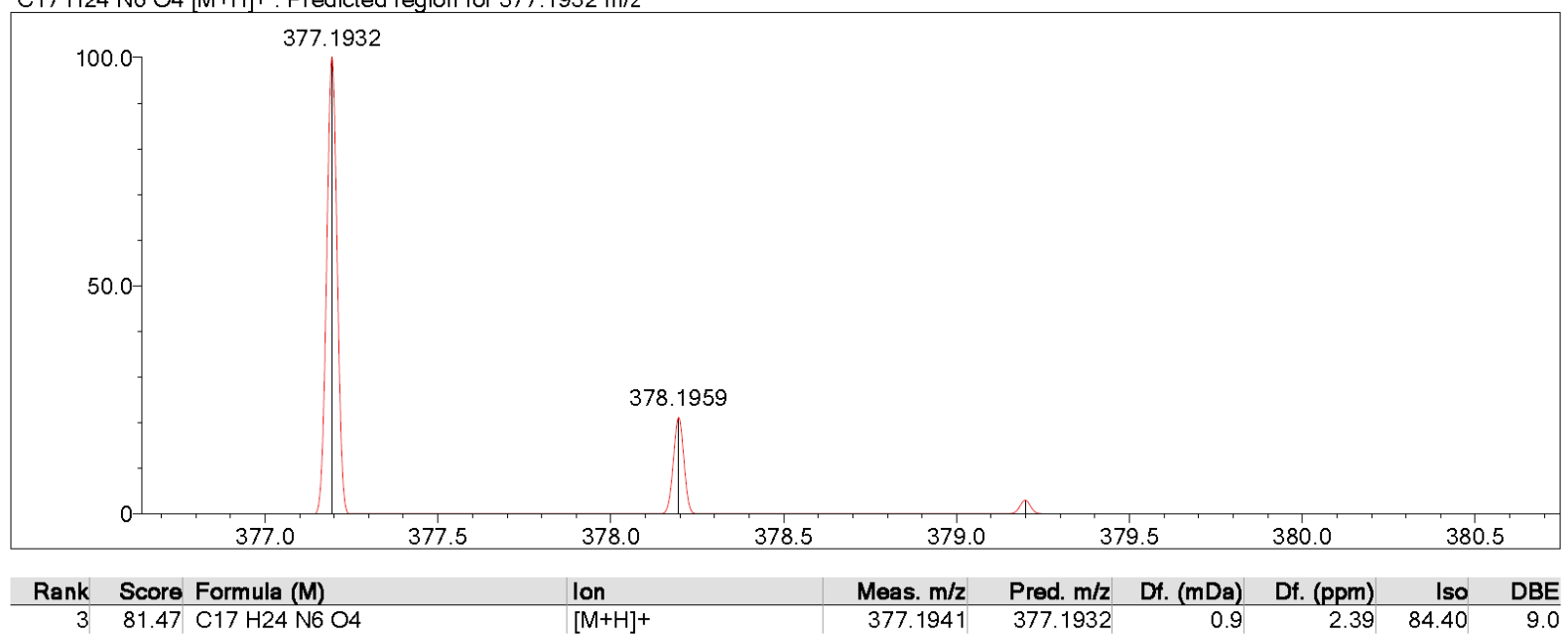




\begin{tabular}{|c|c|c|c|c|c|c|c|c|c|c|c|}
\hline Elmt & Val. & Min & Max & Elmt & Val. & Min & Max & Elmt & Val. & Min & Max \\
\hline $\mathrm{H}$ & 1 & 0 & 30 & $N$ & 3 & 0 & 8 & $P$ & 3 & 0 & 0 \\
\hline B & 3 & 0 & 0 & 0 & 2 & 0 & 5 & $S$ & 2 & 0 & 1 \\
\hline C & 4 & 0 & 25 & $\mathrm{~F}$ & 1 & 0 & 3 & $\mathrm{Cl}$ & 1 & 0 & 3 \\
\hline
\end{tabular}

Error Margin (ppm): 20 HC Ratio: unlimited

Max Isotopes: all

DBE Range: $-1.0-1000.0$

Apply N Rule: no

Isotope RI (\%): 1.00

MSn Logic Mode: AND \begin{tabular}{c|c|rr} 
Elmt & Val. & Min & Max \\
\hline $\mathrm{Se}$ & 2 & 0 & 0 \\
$\mathrm{Br}$ & 1 & 0 & 0 \\
$\mathrm{I}$ & 3 & 0 & 0
\end{tabular}

Use Adduct

$\mathrm{Ha}$

Electron lons: both

Use MSn Info: no

Isotope Res: 10000

Max Results: 40

Event\#: $1 \mathrm{MS}(\mathrm{E}+)$ Ret. Time : 1.002 Scan\# : 201

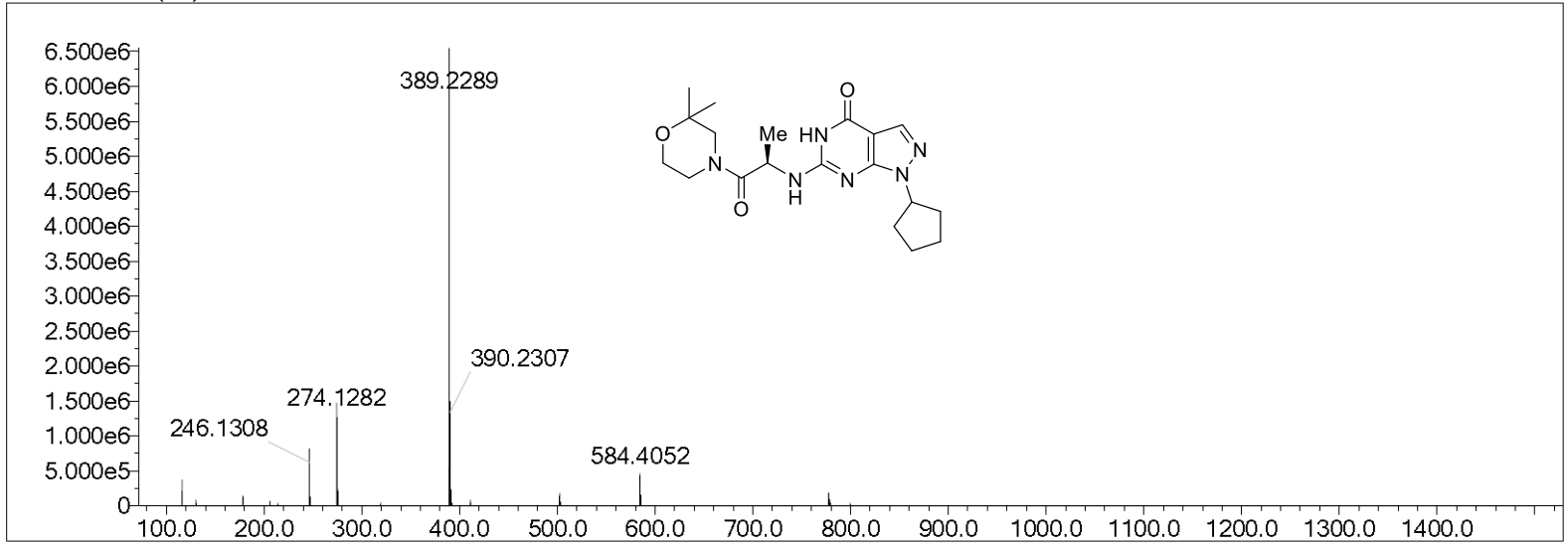

Measured region for $389.2289 \mathrm{~m} / \mathrm{z}$

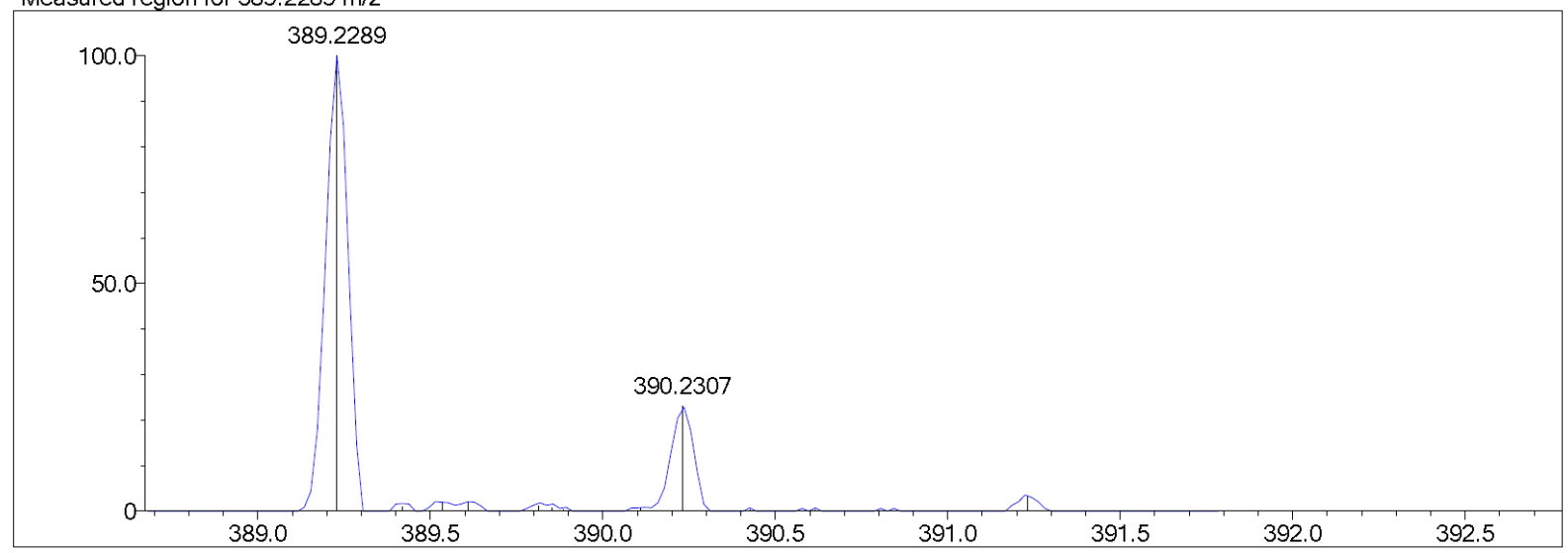

$\mathrm{C} 19 \mathrm{H} 28 \mathrm{N6} \mathrm{O} 3[\mathrm{M}+\mathrm{H}]+$ : Predicted region for $389.2296 \mathrm{~m} / \mathrm{z}$

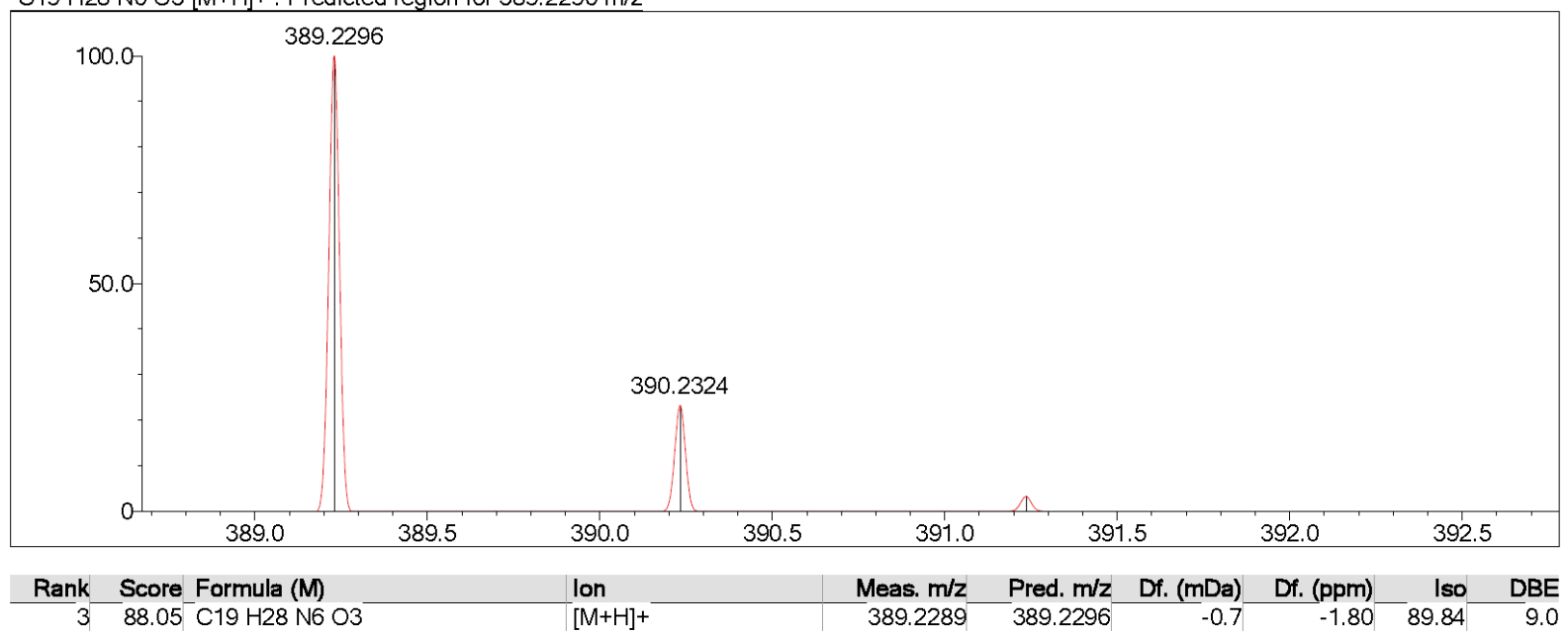




\begin{tabular}{c|r|r|r} 
Elmt & Val. & Min & Max \\
\hline $\mathrm{H}$ & 1 & 0 & 40 \\
$\mathrm{~B}$ & 3 & 0 & 0 \\
$\mathrm{C}$ & 4 & 0 & 40 \\
$\mathrm{~N}$ & 3 & 0 & 8
\end{tabular}

Error Margin (ppm): 30 HC Ratio: unlimited

Max Isotopes: all

MSn Iso RI (\%): 75.00

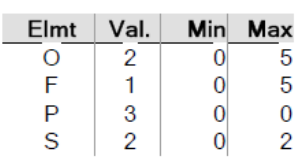

\begin{tabular}{c|c|r|r} 
Elmt & Val. & Min & Max \\
\hline $\mathrm{Cl}$ & 1 & 0 & 5 \\
$\mathrm{Se}$ & 2 & 0 & 0 \\
$\mathrm{Br}$ & 1 & 0 & 5 \\
$\mathrm{I}$ & 3 & 0 & 0
\end{tabular}

DBE Range: $-1.0-1000.0$ Apply N Rule: no Isotope RI (\%): 1.00

MSn Logic Mode: AND
Use Adduct

$\mathrm{H}$

K

Electron lons: both

Use MSn Info: no

Isotope Res: 10000

Max Results: 40

Event\#: $1 \mathrm{MS}\left(\mathrm{E}^{+}\right)$Ret. Time : 0.922 Scan\# : 185

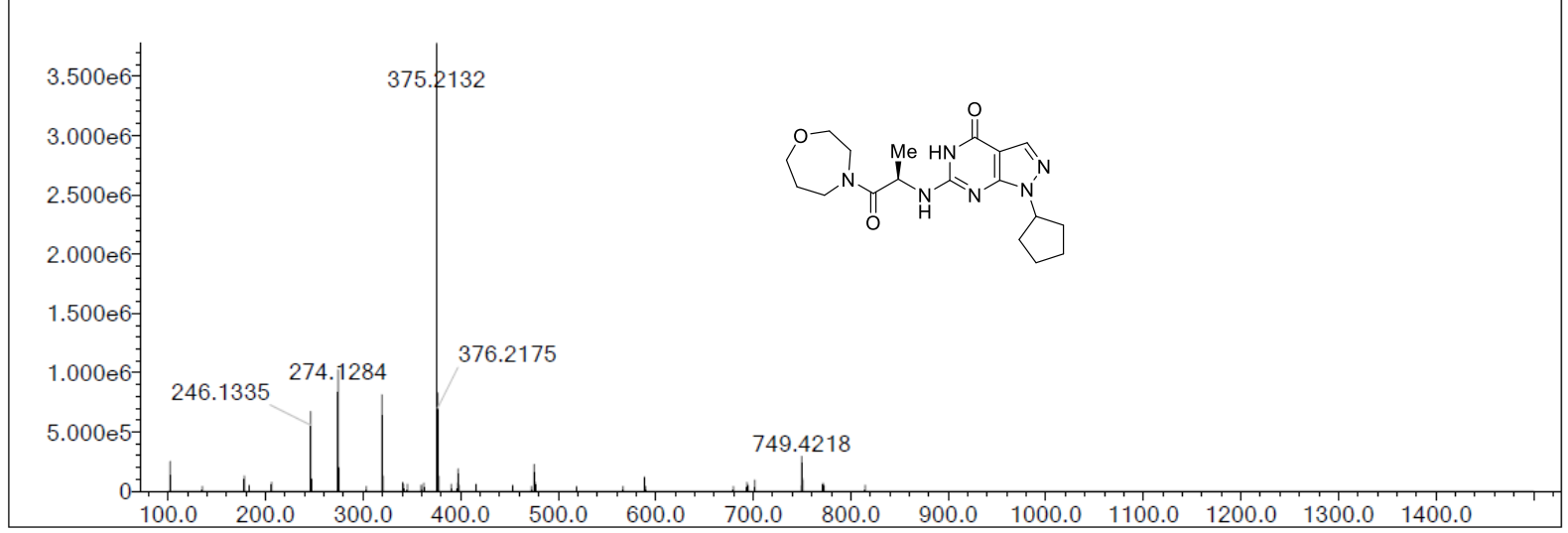

Measured region for $375.2132 \mathrm{~m} / \mathrm{z}$

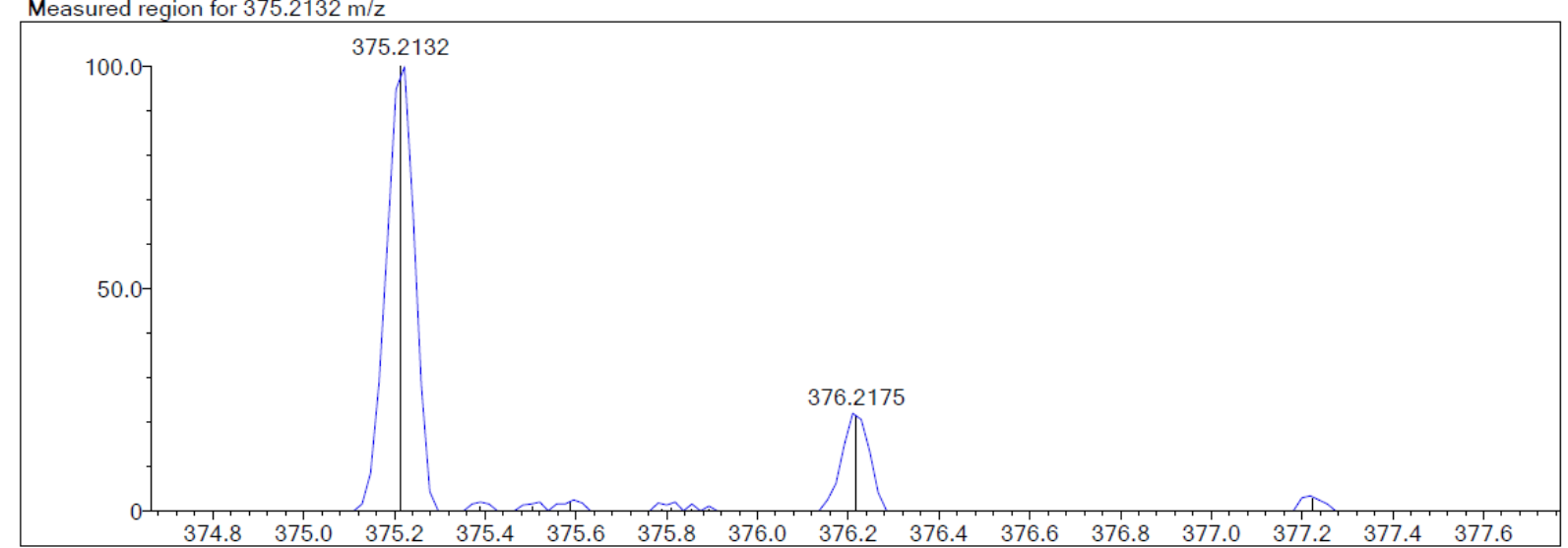

$\mathrm{C} 18 \mathrm{H} 26 \mathrm{N6} \mathrm{O} 3[\mathrm{M}+\mathrm{H}]+$ : Predicted region for $375.2139 \mathrm{~m} / \mathrm{z}$

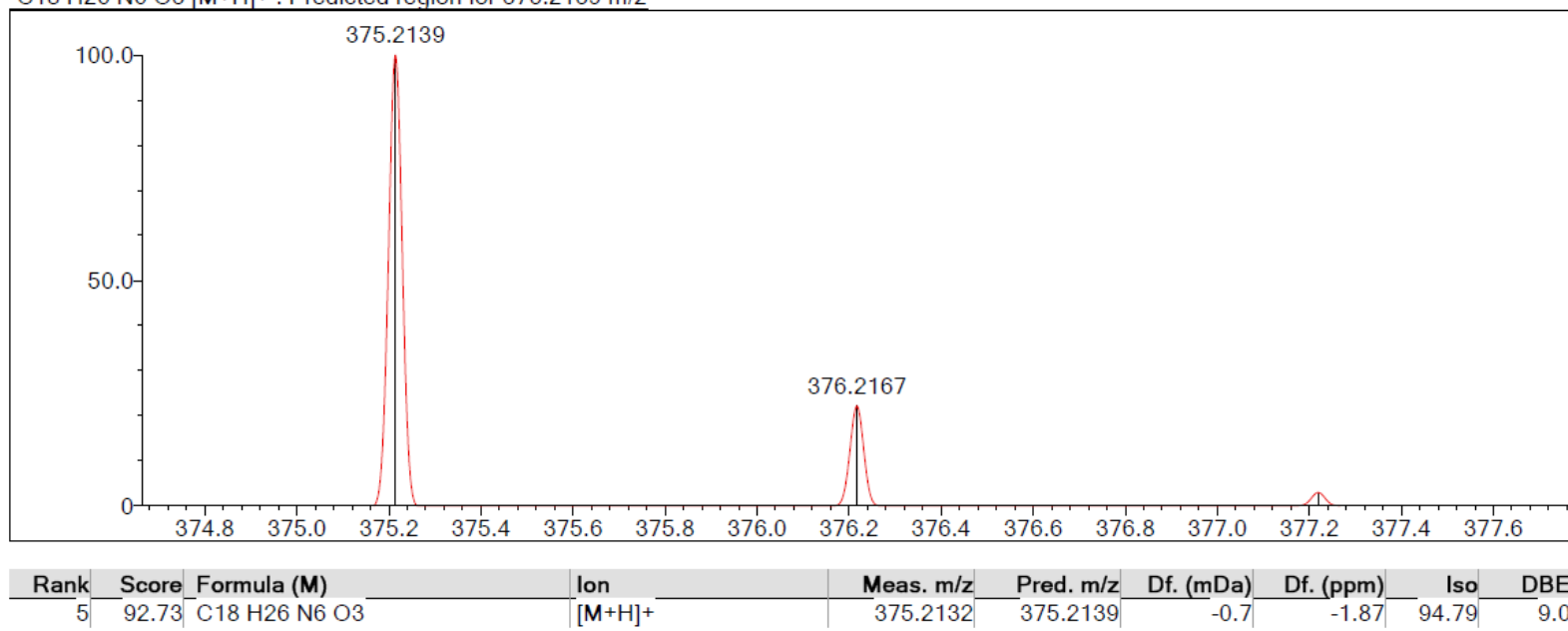




\begin{tabular}{c|r|rr} 
Elmt & Val. & Min & Max \\
\hline $\mathrm{H}$ & 1 & 12 & 38 \\
$2 \mathrm{H}$ & 1 & 0 & 0 \\
$\mathrm{~B}$ & 3 & 0 & 0 \\
$\mathrm{C}$ & 4 & 12 & 20 \\
$\mathrm{~N}$ & 3 & 0 & 8 \\
$\mathrm{O}$ & 2 & 1 & 5
\end{tabular}

Error Margin (ppm): 30 HC Ratio: unlimited

Max Isotopes: all MSn Iso RI (\%): 75.00

\begin{tabular}{c|r|r|r} 
Elmt & Val. & Min & Max \\
\hline $\mathrm{F}$ & 1 & 0 & 4 \\
$\mathrm{Si}$ & 4 & 0 & 0 \\
$\mathrm{P}$ & 3 & 0 & 0 \\
$\mathrm{~S}$ & 2 & 0 & 2 \\
$\mathrm{Cl}$ & 1 & 0 & 2 \\
$\mathrm{Se}$ & 2 & 0 & 0
\end{tabular}

\begin{tabular}{c|r|r|r} 
Elmt & Val. & Min & Max \\
\hline $\mathrm{Br}$ & 1 & 0 & 0 \\
$\mathrm{I}$ & 3 & 0 & 0
\end{tabular}

DBE Range: $-1.0-1000.0$ Apply N Rule: no

Isotope RI (\%): 1.00

MSn Logic Mode: AND

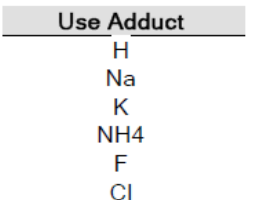

Electron lons: both

Use MSn Info: no

Isotope Res: 10000

Max Results: 40

Event\#: $1 \mathrm{MS}\left(\mathrm{E}^{+}\right)$Ret. Time : 0.732 Scan\# : 147

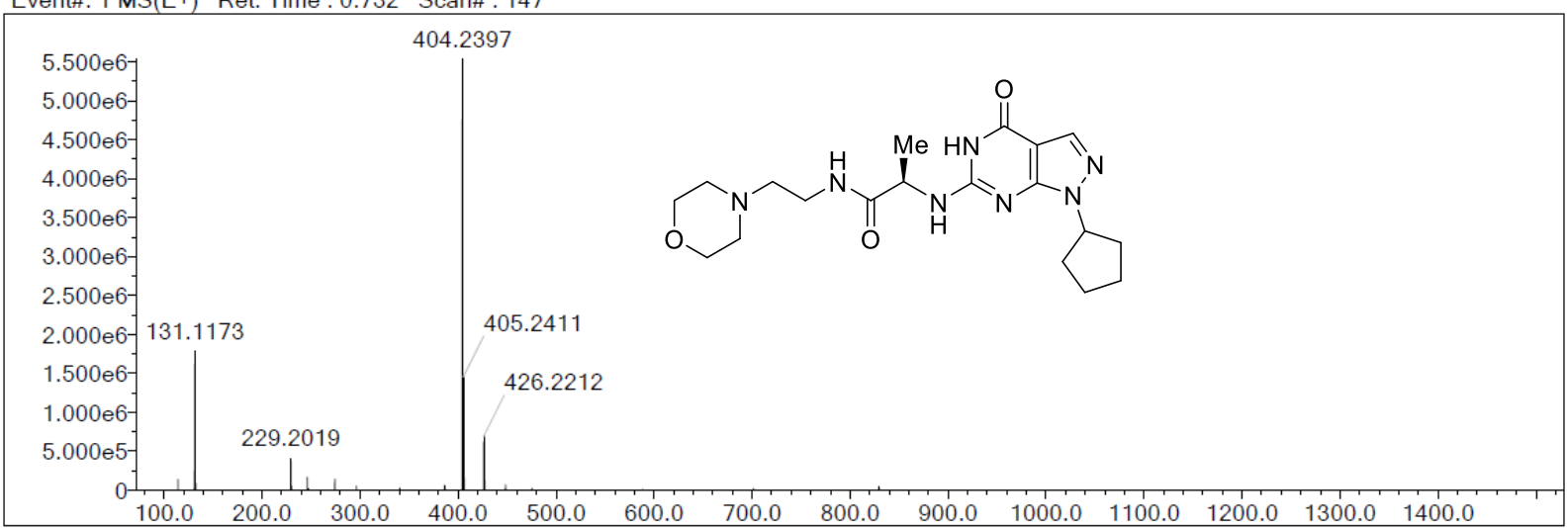

Measured region for $404.2397 \mathrm{~m} / \mathrm{z}$

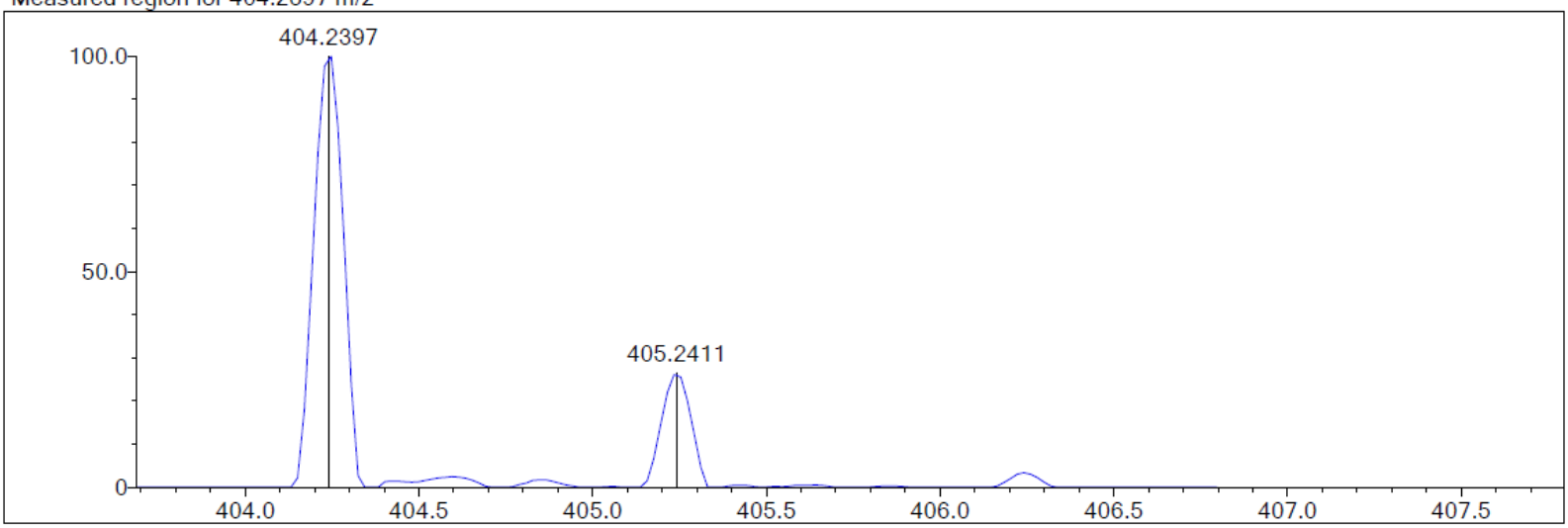

$\mathrm{C} 19 \mathrm{H} 29 \mathrm{~N} 7 \mathrm{O} 3[\mathrm{M}+\mathrm{H}]+$ : : Predicted region for $404.2405 \mathrm{~m} / \mathrm{z}$

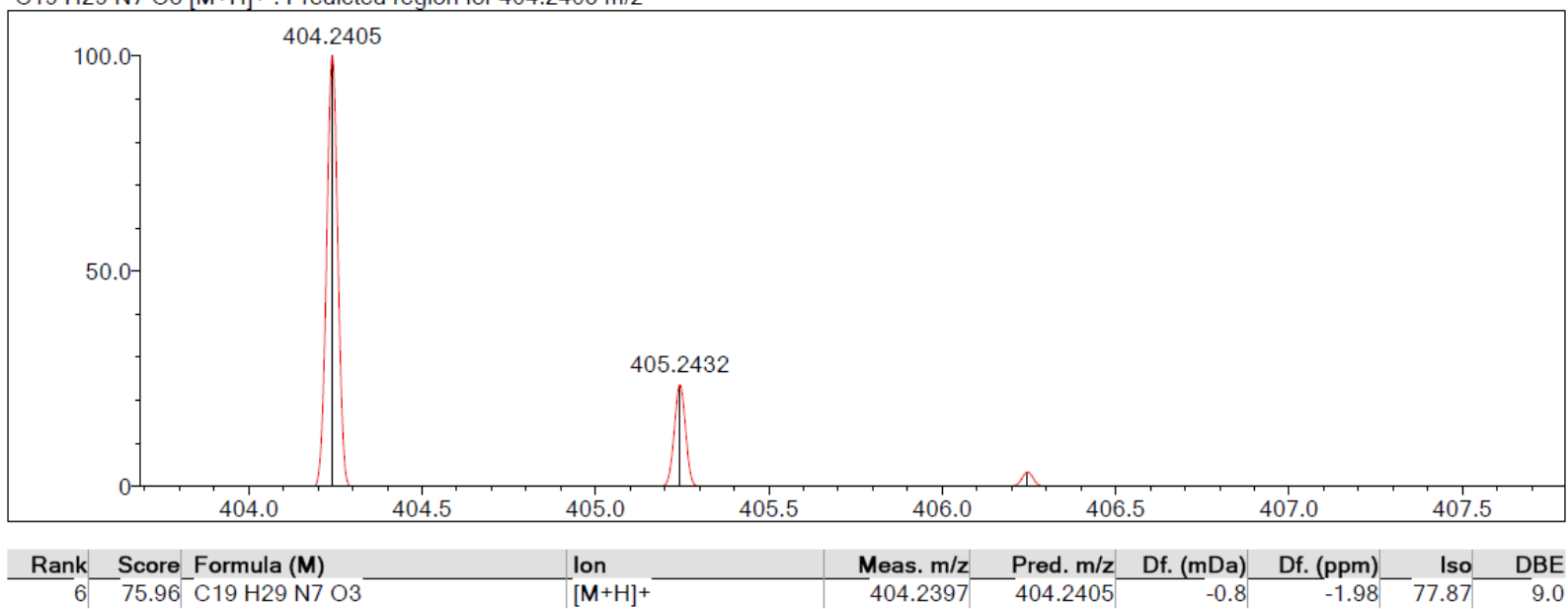




\begin{tabular}{|c|c|c|c|c|c|c|c|c|c|c|c|}
\hline Elmt & Val. & Min & Max & Elmt & Val. & Min & Max & Elmt & Val. & Min & Max \\
\hline $\mathrm{H}$ & 1 & 12 & 38 & $\mathrm{~N}$ & 3 & 0 & 8 & $P$ & 3 & 0 & 0 \\
\hline $2 \mathrm{H}$ & 1 & 0 & 0 & 0 & 2 & 1 & 5 & $S$ & 2 & 0 & 2 \\
\hline B & 3 & 0 & 0 & $F$ & 1 & 0 & 2 & $\mathrm{Cl}$ & 1 & 0 & 2 \\
\hline C & 4 & 12 & 20 & $\mathrm{Si}$ & 4 & 0 & 0 & Se & 2 & 0 & 0 \\
\hline
\end{tabular}

Error Margin (ppm): 30

HC Ratio: unlimited

DBE Range: $-1.0-1000.0$

Apply N Rule: no

Isotope RI (\%): 1.00
MSn Logic Mode: AND

Max Isotopes: all

\begin{tabular}{c|c|r|r} 
Elmt & Val. & Min & Max \\
\hline $\mathrm{Br}$ & 1 & 0 & 0
\end{tabular}

Use Adduct

$0 \quad \mathrm{H}$

$\mathrm{Na}$

$\mathrm{Cl}$

MSn Iso RI (\%): 75.00

Electron lons: both

Use MSn Info: no

Isotope Res: 10000

Max Results: 40

Event\#: $1 \mathrm{MS}(\mathrm{E}+)$ Ret. Time: 0.982 Scan\# : 197

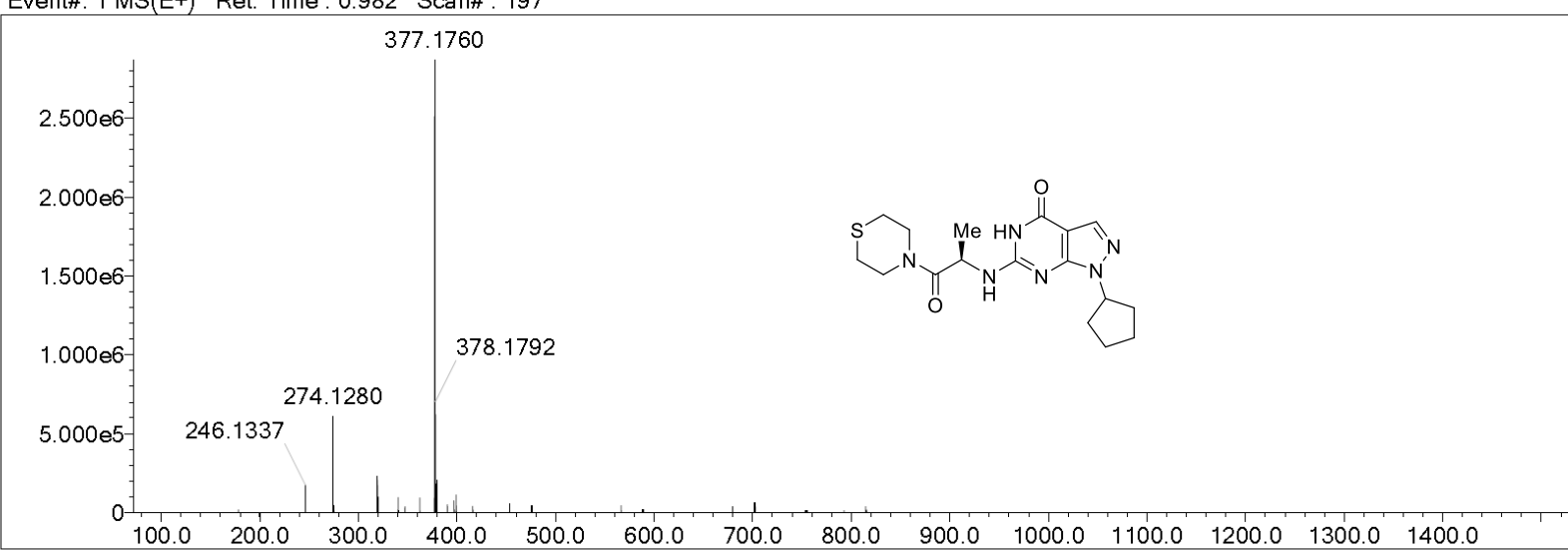

Measured region for $377.1760 \mathrm{~m} / \mathrm{z}$

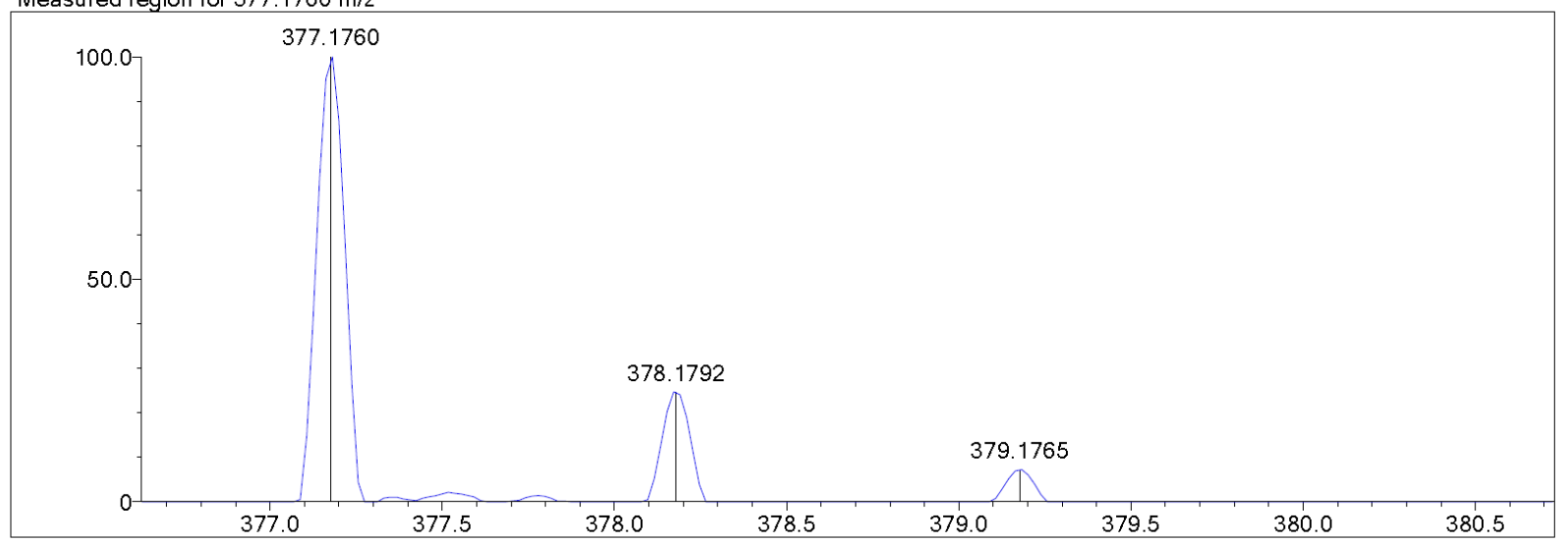

C17 H24 N6 O2 S [M+H]+ : Predicted region for $377.1754 \mathrm{~m} / \mathrm{z}$

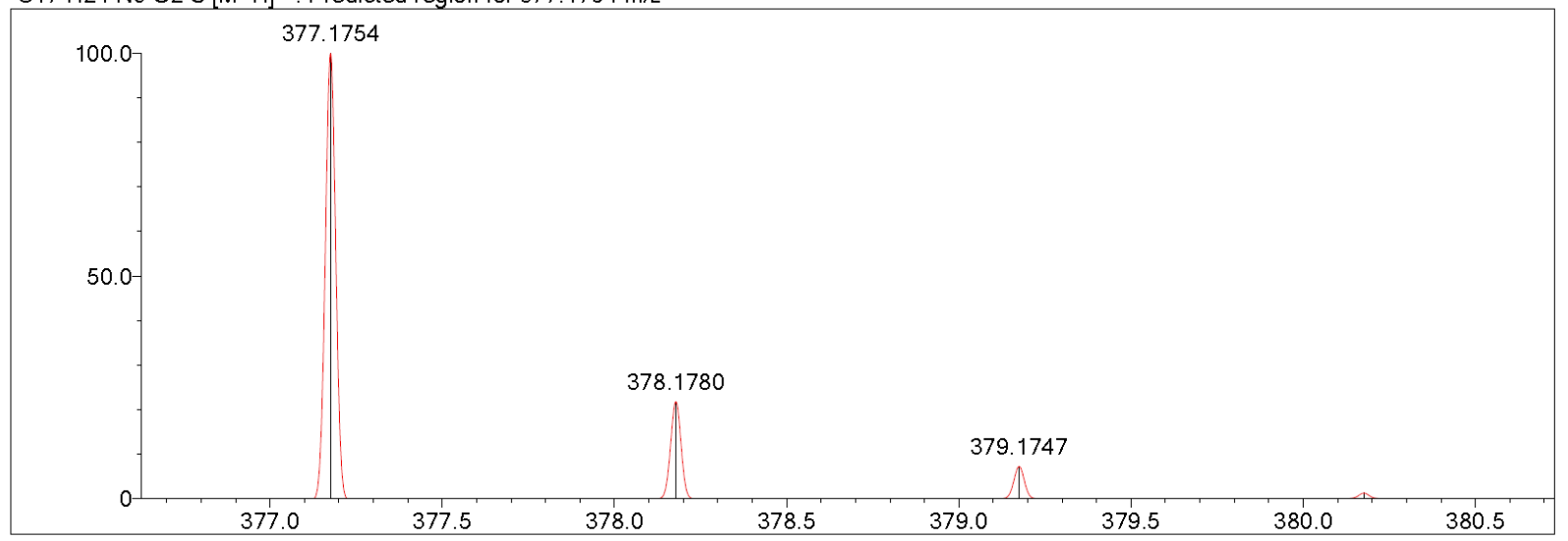

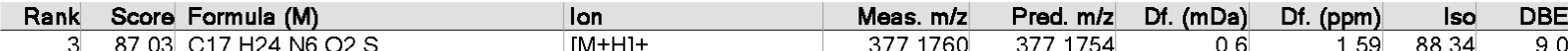




\begin{tabular}{c|r|r|r} 
Elmt & Val. & Min & Max \\
\hline $\mathrm{H}$ & 1 & 12 & 38 \\
$2 \mathrm{H}$ & 1 & 0 & 0 \\
$\mathrm{~B}$ & 3 & 0 & 0 \\
$\mathrm{C}$ & 4 & 12 & 20
\end{tabular}

Error Margin (ppm): 30 HC Ratio: unlimited

Max Isotopes: all

MSn Iso RI (\%): 75.00

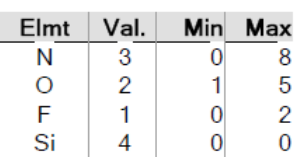

\begin{tabular}{c|r|r|r} 
Elmt & Val. & Min & Max \\
\hline $\mathrm{P}$ & 3 & 0 & 0 \\
$\mathrm{~S}$ & 2 & 0 & 2 \\
$\mathrm{Cl}$ & 1 & 0 & 2 \\
$\mathrm{Se}$ & 2 & 0 & 0
\end{tabular}

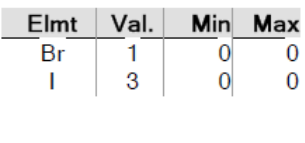

Use Adduct

$\mathrm{H}$

$\mathrm{Na}$

$\mathrm{K}$
DBE Range: -1.0 - 1000.0

Apply N Rule: no

Isotope RI (\%): 1.00

MSn Logic Mode: AND
Electron lons: both

Use MSn Info: no

Isotope Res: 10000

Max Results: 40

Event\#: $1 \mathrm{MS}\left(\mathrm{E}^{+}\right)$Ret. Time : 1.157 Scan\# : 232

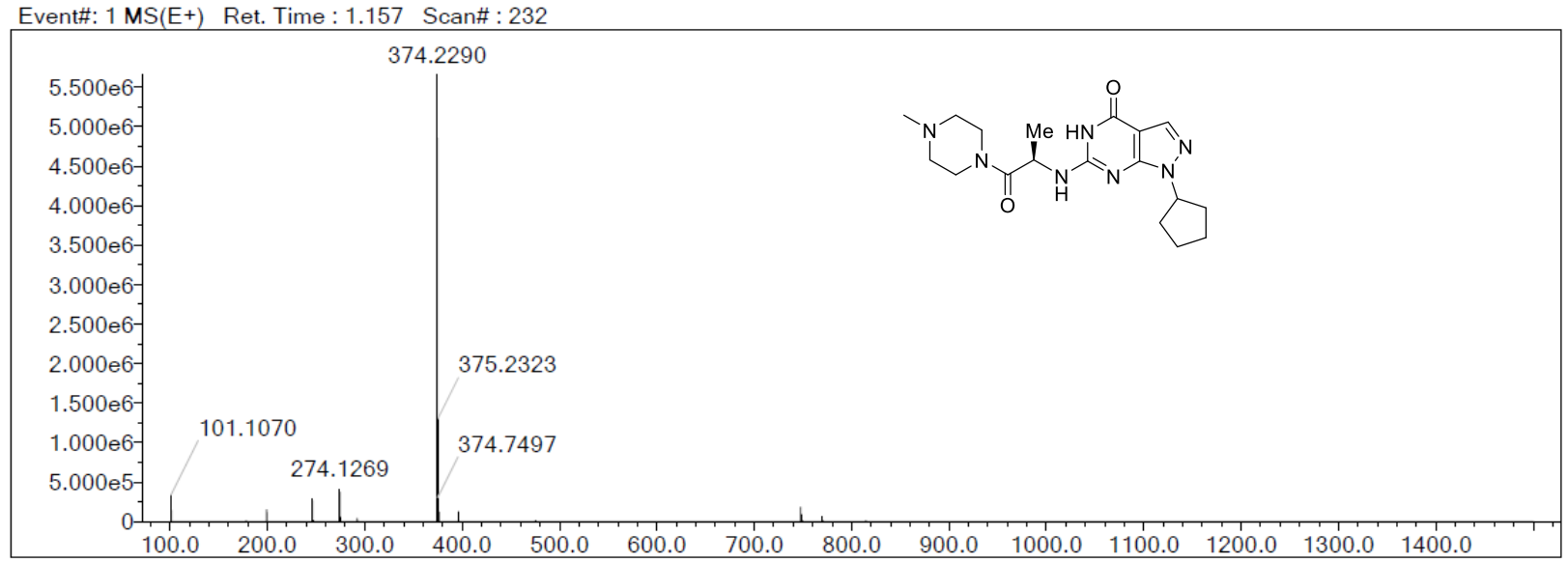

Measured region for $374.2290 \mathrm{~m} / \mathrm{z}$

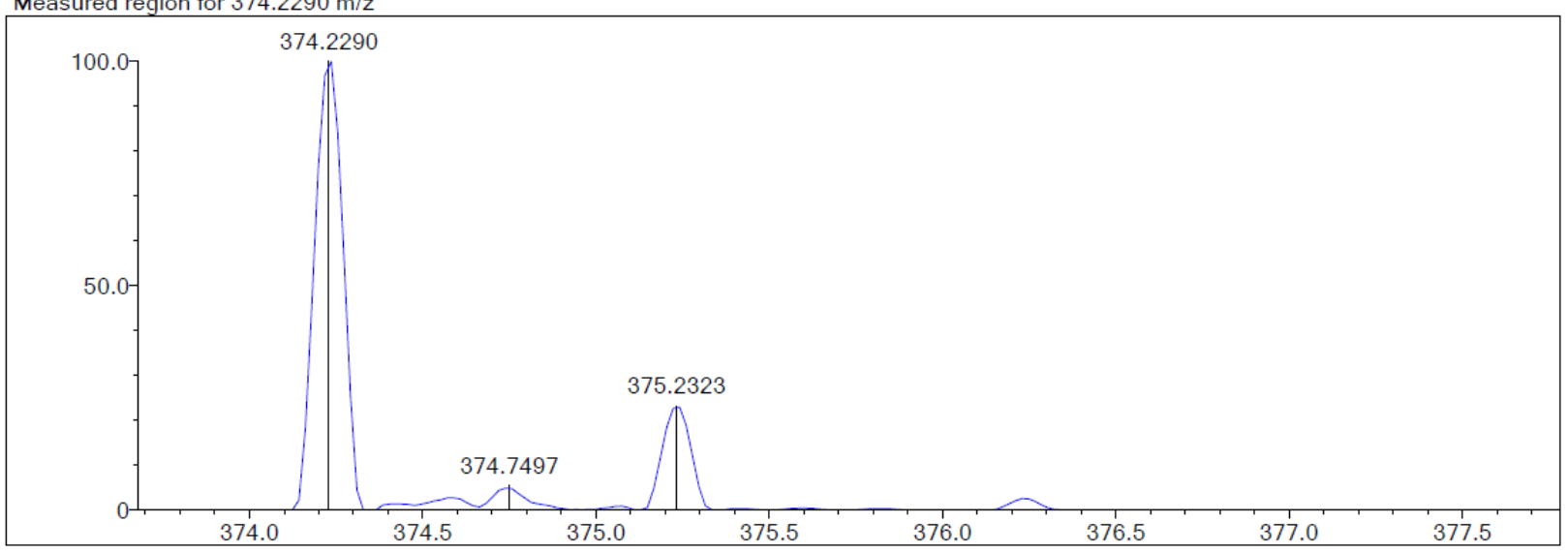

$\mathrm{C} 18 \mathrm{H} 27 \mathrm{~N} 7 \mathrm{O} 2[\mathrm{M}+\mathrm{H}]+$ : Predicted region for $374.2299 \mathrm{~m} / \mathrm{z}$

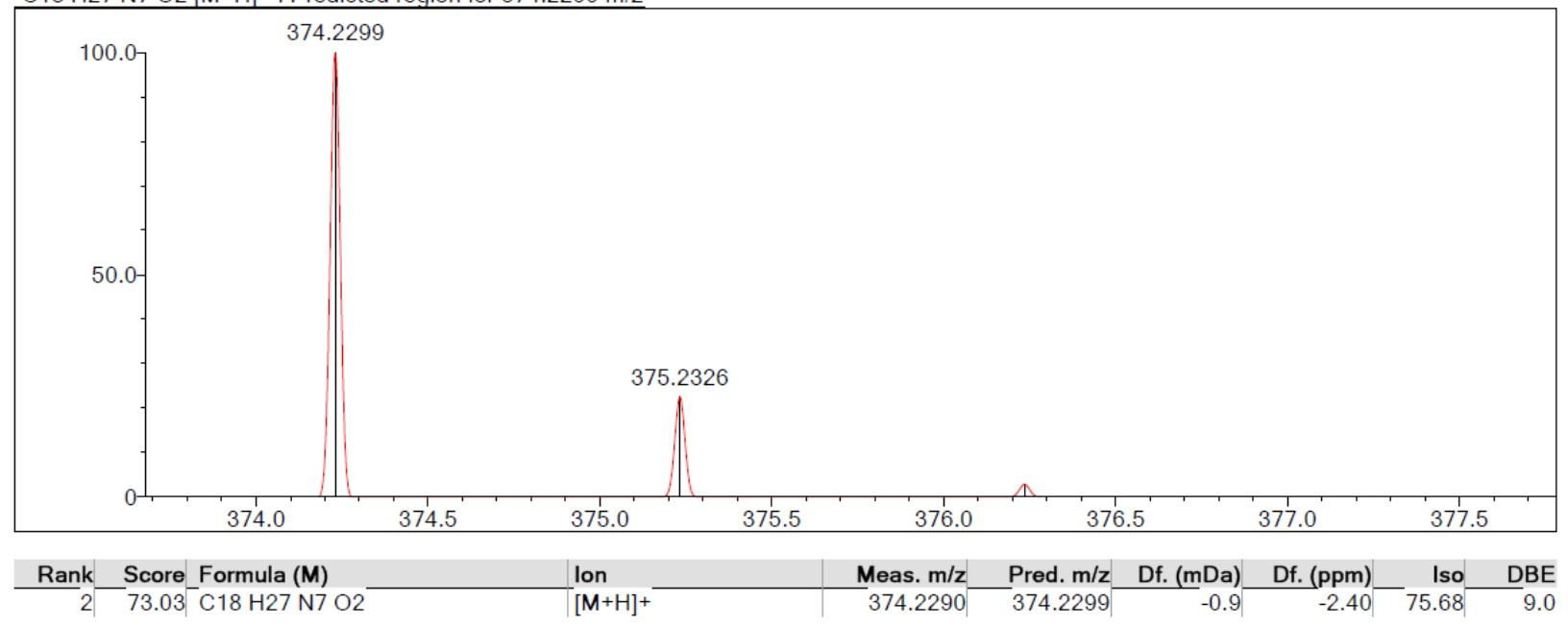




\begin{tabular}{c|r|r|r} 
Elmt & Val. & Min & Max \\
\hline $\mathrm{H}$ & 1 & 12 & 38 \\
$2 \mathrm{H}$ & 1 & 0 & 0 \\
$\mathrm{~B}$ & 3 & 0 & 0 \\
$\mathrm{C}$ & 4 & 12 & 20 \\
$\mathrm{~N}$ & 3 & 0 & 8 \\
$\mathrm{O}$ & 2 & 1 & 5
\end{tabular}

Error Margin (ppm): 30 HC Ratio: unlimited Max Isotopes: all MSn Iso RI (\%): 75.00

\begin{tabular}{c|r|r|r} 
Elmt & Val. & Min & Max \\
\hline $\mathrm{F}$ & 1 & 0 & 4 \\
$\mathrm{Si}$ & 4 & 0 & 0 \\
$\mathrm{P}$ & 3 & 0 & 0 \\
$\mathrm{~S}$ & 2 & 0 & 2 \\
$\mathrm{Cl}$ & 1 & 0 & 2 \\
$\mathrm{Se}$ & 2 & 0 & 0
\end{tabular}

DBE Range: -1.0 - 1000.0

Apply N Rule: no

Isotope RI (\%): 1.00

MSn Logic Mode: AND
Use Adduct

$\mathrm{H}$

$\mathrm{Na}$

$\mathrm{NH} 4$

$\mathrm{F}$

Electron lons: both

Use MSn Info: no

Isotope Res: 10000

Max Results: 40

Event\#: $1 \mathrm{MS}\left(\mathrm{E}^{+}\right)$Ret. Time : 1.022 Scan\# : 205

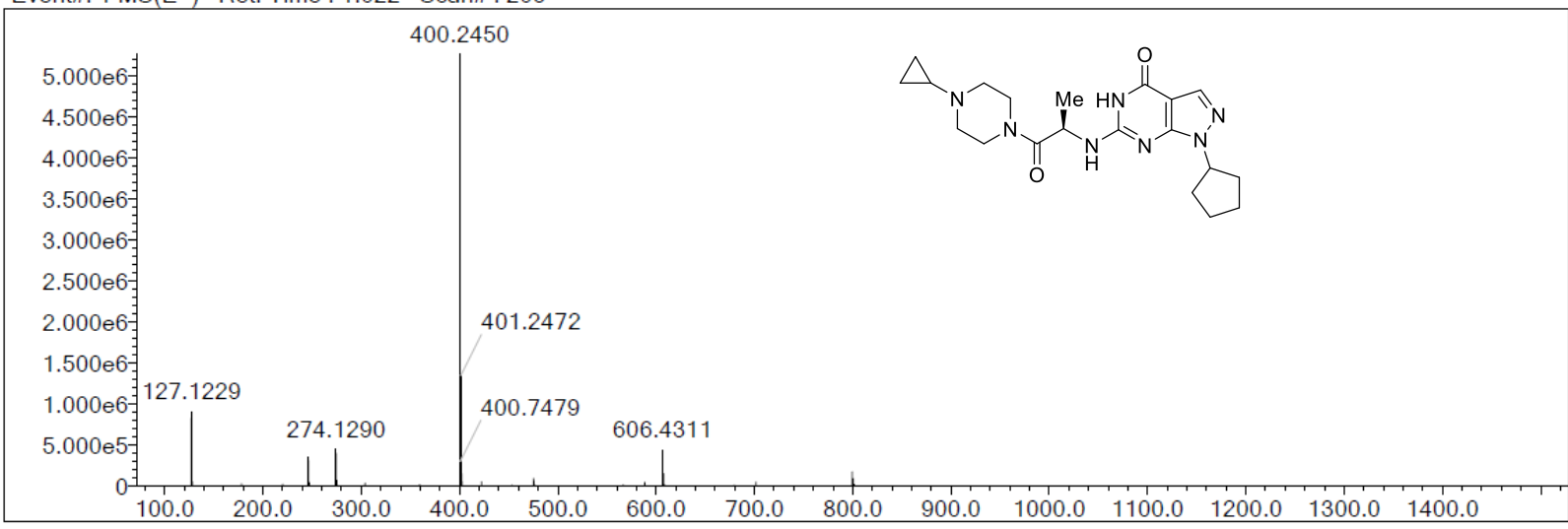

Measured region for $400.2450 \mathrm{~m} / \mathrm{z}$

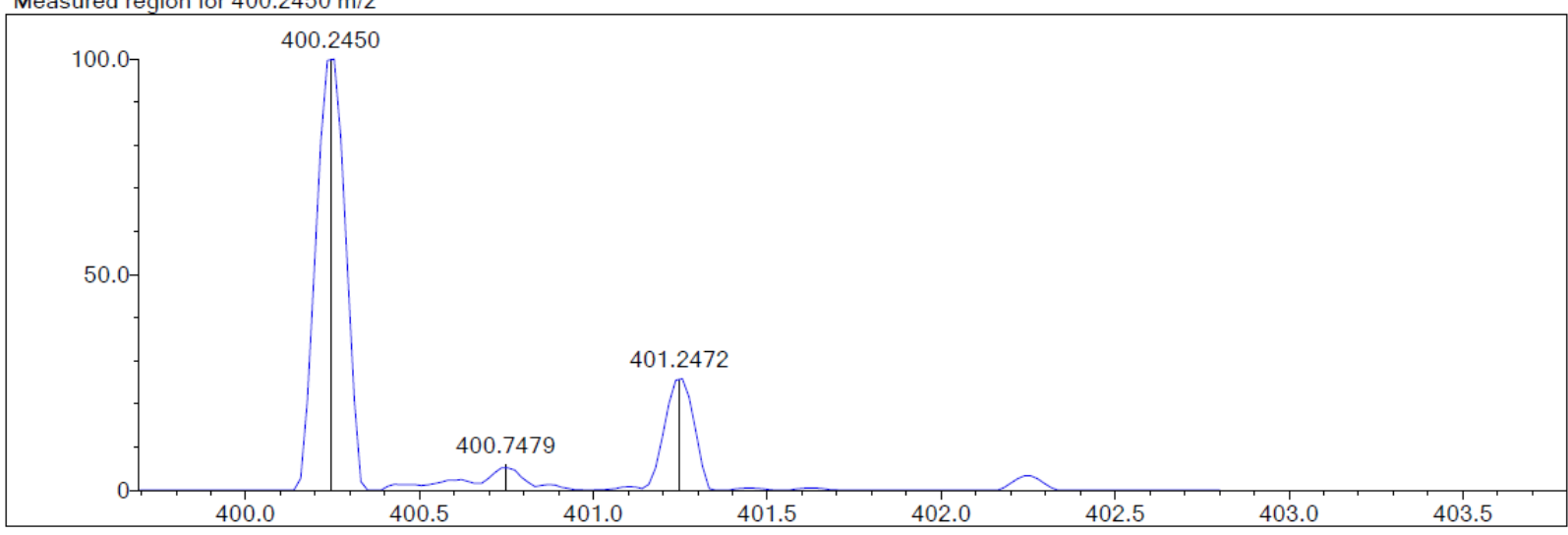

$\mathrm{C} 20 \mathrm{H} 29 \mathrm{~N} 7 \mathrm{O} 2[\mathrm{M}+\mathrm{H}]+$ : Predicted region for $400.2455 \mathrm{~m} / \mathrm{z}$

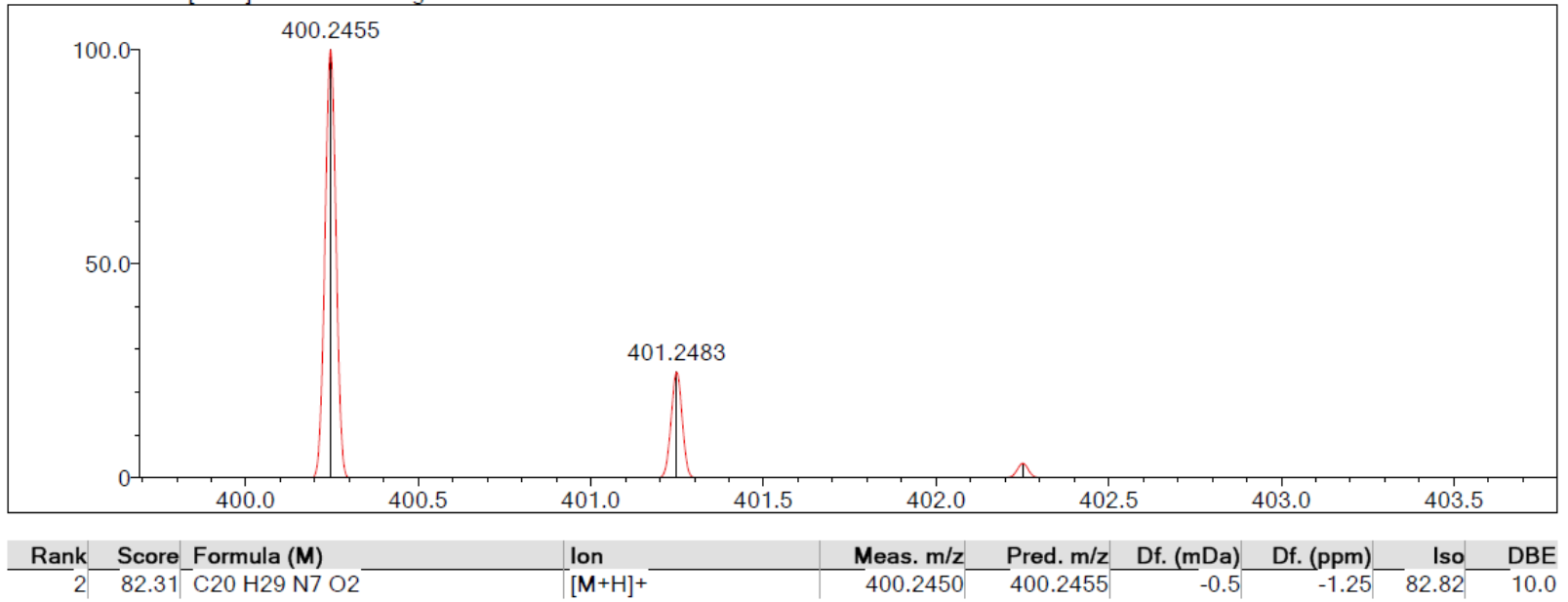




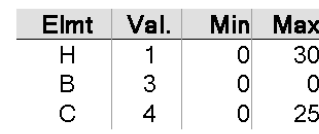

Error Margin (ppm): 20 HC Ratio: unlimited Max Isotopes: all MSn Iso RI (\%): 75.00

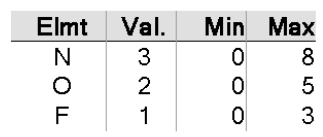

\begin{tabular}{c|c|r|r}
\hline Elmt & Val. & Min & Max \\
\hline $\mathrm{P}$ & 3 & 0 & 0 \\
$\mathrm{~S}$ & 2 & 0 & 1 \\
$\mathrm{Cl}$ & 1 & 0 & 3
\end{tabular}

DBE Range: $-1.0-1000.0$

Apply N Rule: no

Isotope RI (\%): 1.00

MSn Logic Mode: AND

\begin{tabular}{c|c|r|r} 
Elmt & Val. & Min & Max \\
\hline $\mathrm{Se}$ & 2 & 0 & 0 \\
$\mathrm{Br}$ & 1 & 0 & 0 \\
$\mathrm{I}$ & 3 & 0 & 0
\end{tabular}

Use Adduct

$\mathrm{H}$

$\mathrm{Na}$

Event\#: $1 \mathrm{MS}(\mathrm{E}+)$ Ret. Time : 1.022 Scan\# : 205

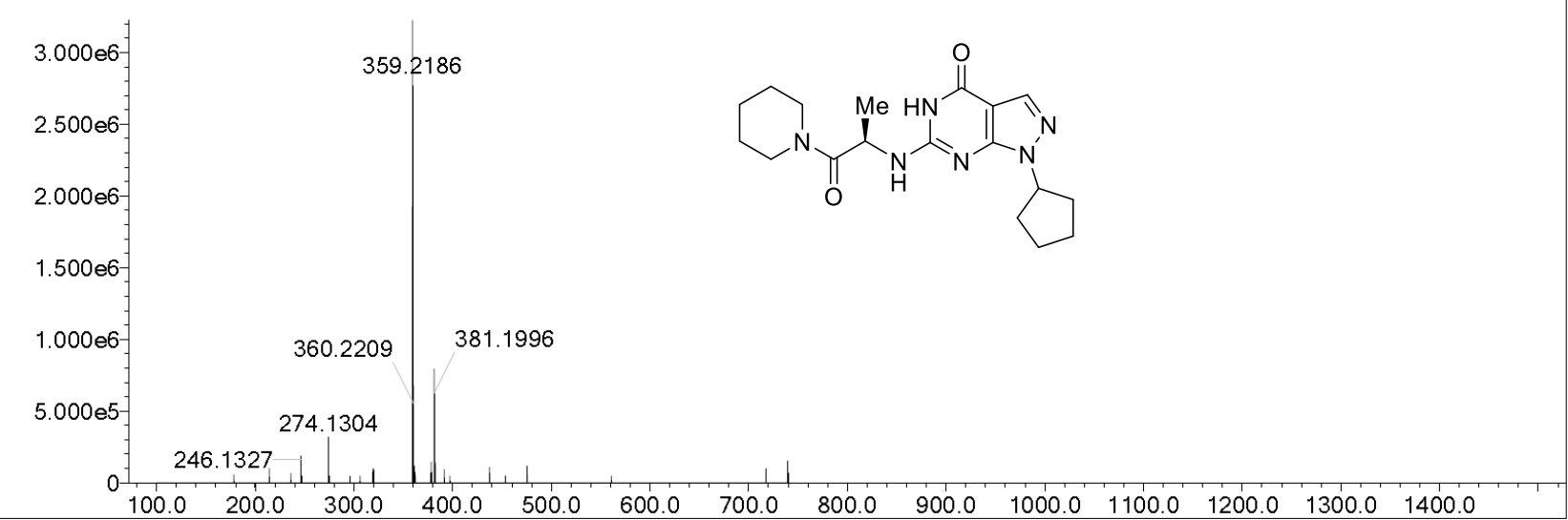

Measured region for $359.2186 \mathrm{~m} / \mathrm{z}$

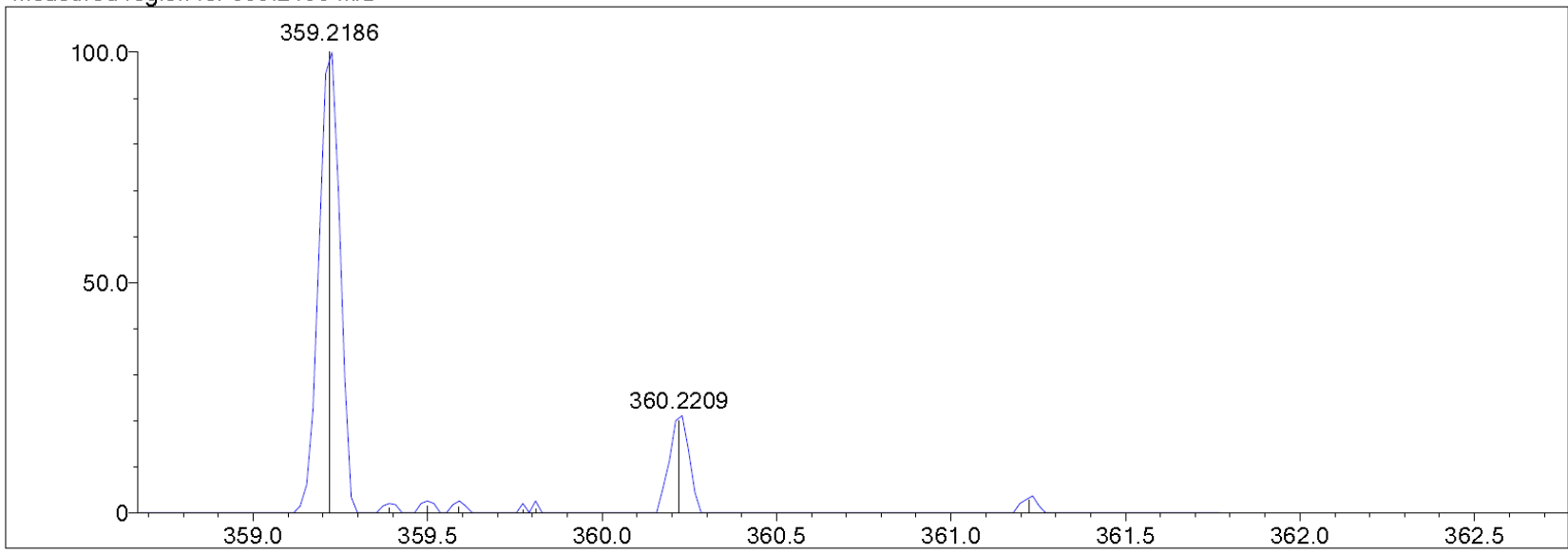

C18 H26 N6 O2 [M+H]+ : Predicted region for $359.2190 \mathrm{~m} / \mathrm{z}$

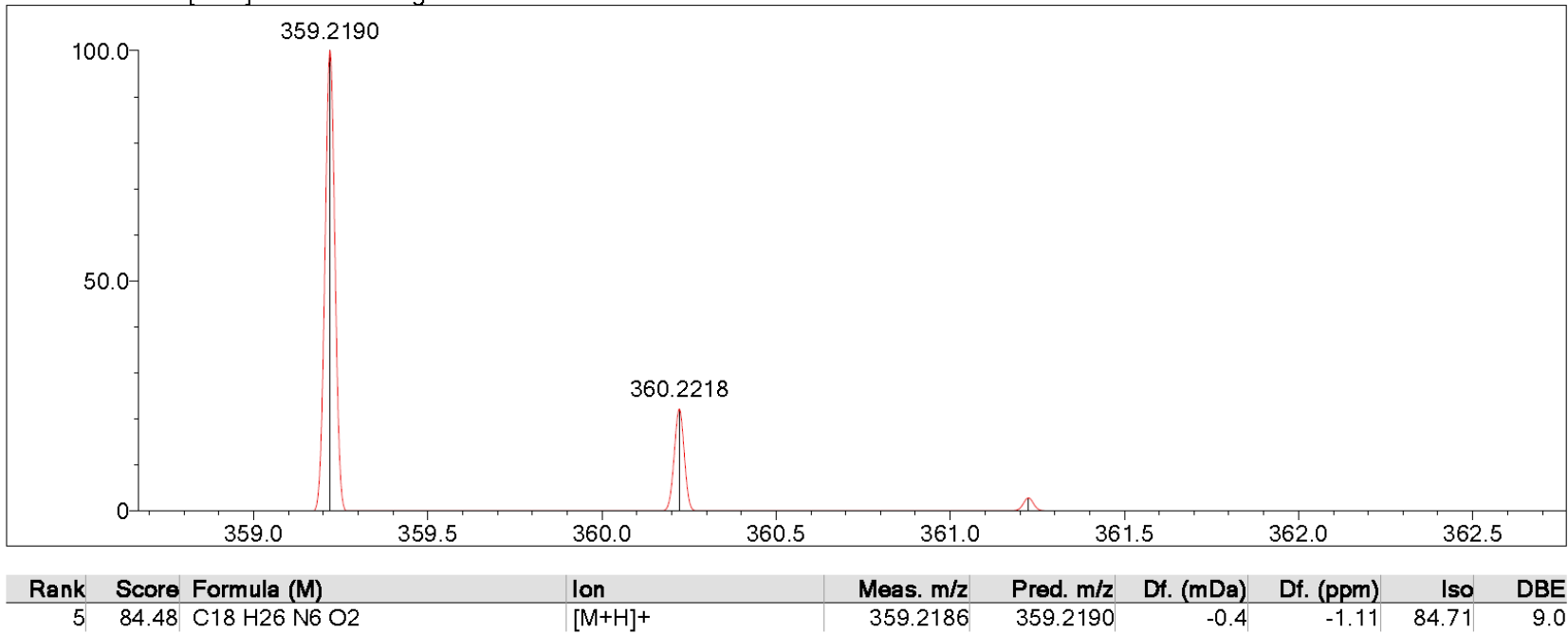




\begin{tabular}{c|c|r|r}
\hline Elmt & Val. & Min & Max \\
\hline H & 1 & 0 & 40 \\
B & 3 & 0 & 0 \\
C & 4 & 0 & 40 \\
N & 3 & 0 & 8
\end{tabular}

Error Margin (ppm): 30 HC Ratio: unlimited Max Isotopes: all MSn Iso RI (\%): 75.00

\begin{tabular}{c|c|r|r|r|r|r|r|}
\hline Elmt & Val. & Min & Max & Elmt & Val. & Min & Max \\
\hline $\mathrm{O}$ & 2 & 0 & 5 & $\mathrm{Cl}$ & 1 & 0 & 5 \\
$\mathrm{~F}$ & 1 & 0 & 5 & $\mathrm{Se}$ & 2 & 0 & 0 \\
$\mathrm{P}$ & 3 & 0 & 0 & $\mathrm{Br}$ & 1 & 0 & 5 \\
$\mathrm{~S}$ & 2 & 0 & 2 & $\mathrm{I}$ & 3 & 0 & 0 \\
\hline
\end{tabular}

DBE Range: -1.0 - 1000.0

Apply N Rule: no

Isotope RI (\%): 1.00

MSn Logic Mode: AND
Use Adduct

$\mathrm{H}$

$\mathrm{Na}$

$\mathrm{K}$

$\mathrm{NH} 4$

Electron lons: both

Use MSn Info: no

Isotope Res: 10000

Max Results: 40

Event\#: $1 \mathrm{MS}(\mathrm{E}+)$ Ret. Time : 0.997 Scan\# : 200

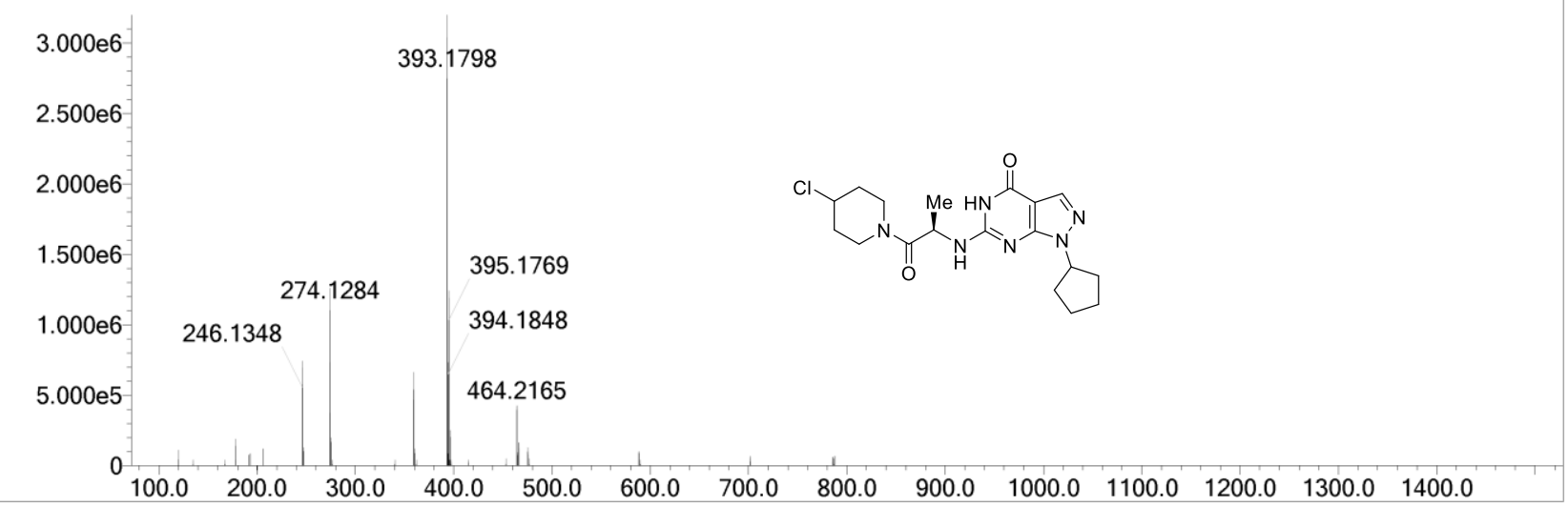

Measured region for $393.1798 \mathrm{~m} / \mathrm{z}$

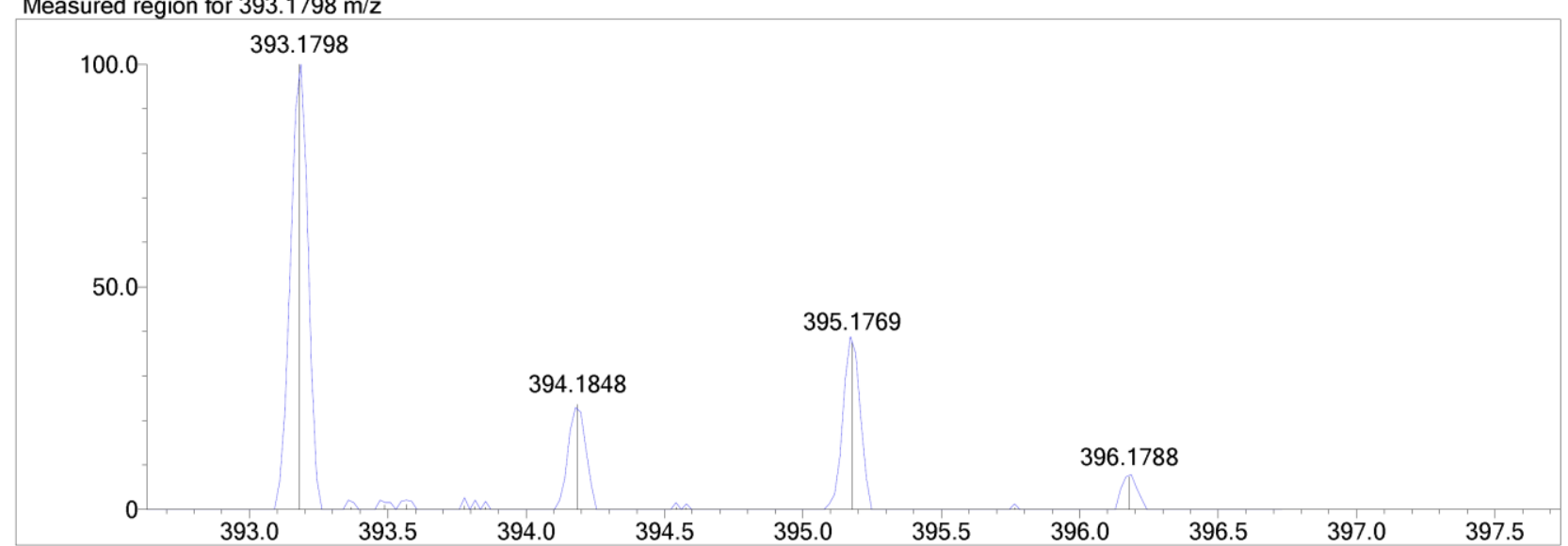

$\mathrm{C} 18 \mathrm{H} 25 \mathrm{~N} 6 \mathrm{O} 2 \mathrm{Cl}[\mathrm{M}+\mathrm{H}]+$ : Predicted region for $393.1800 \mathrm{~m} / \mathrm{z}$

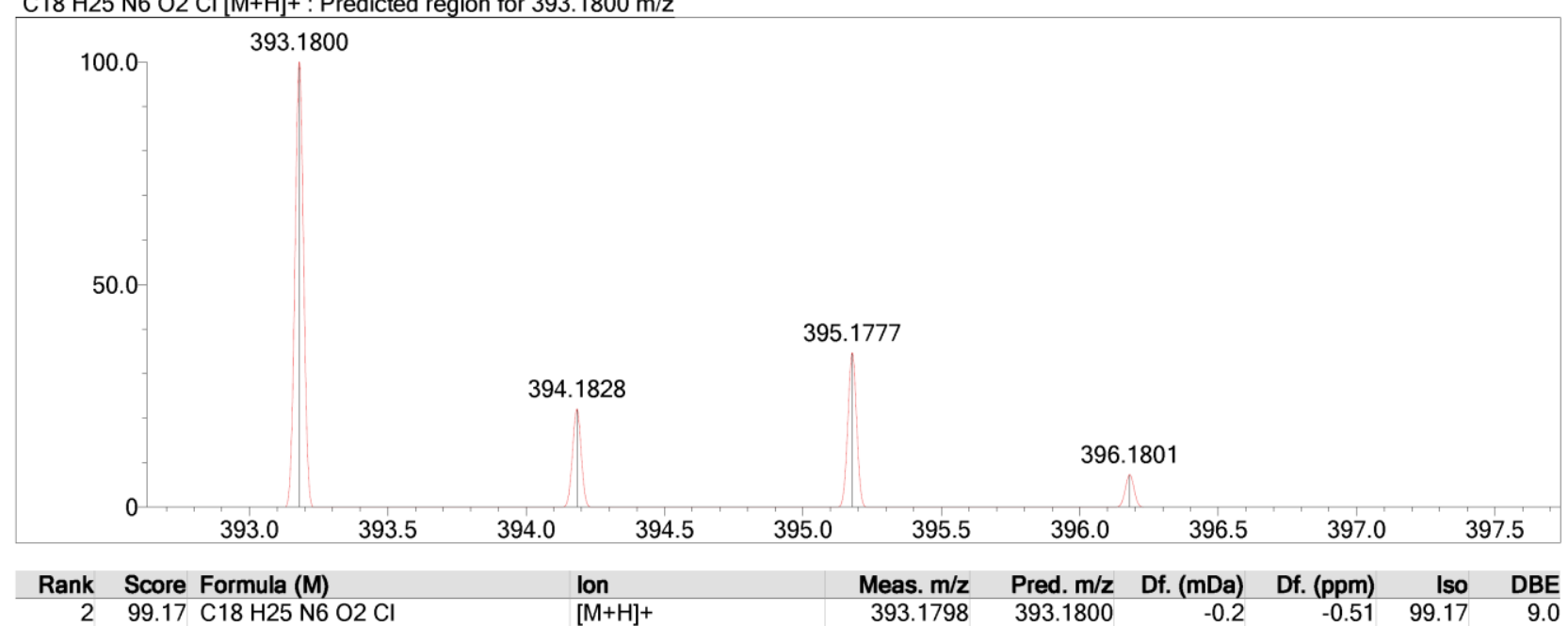




\begin{tabular}{|c|c|c|c|c|c|c|c|c|c|c|c|}
\hline Elmt & Val. & Min & Max & Elmt & Val. & Min & Max & Elmt & Val. & Min & Max \\
\hline $\mathrm{H}$ & 1 & 0 & 30 & $N$ & 3 & 0 & 8 & $P$ & 3 & 0 & 0 \\
\hline B & 3 & 0 & 0 & 0 & 2 & 0 & 5 & $S$ & 2 & 0 & 1 \\
\hline C & 4 & 0 & 25 & $\mathrm{~F}$ & 1 & 0 & 3 & $\mathrm{Cl}$ & 1 & 0 & 3 \\
\hline
\end{tabular}

Error Margin (ppm): 20 HC Ratio: unlimited Max Isotopes: all MSn Iso RI (\%): 75.00

DBE Range: -1.0 - 1000.0

Apply N Rule: no

Isotope RI (\%): 1.00

MSn Logic Mode: AND

\begin{tabular}{c|c|rr} 
Elmt & Val. & Min & Max \\
\hline $\mathrm{Se}$ & 2 & 0 & 0 \\
$\mathrm{Br}$ & 1 & 0 & 0 \\
$\mathrm{I}$ & 3 & 0 & 0
\end{tabular}

Use Adduct $\mathrm{H}$

Na

Event\#: $1 \mathrm{MS}(\mathrm{E}+)$ Ret. Time : 0.977 Scan\# : 196

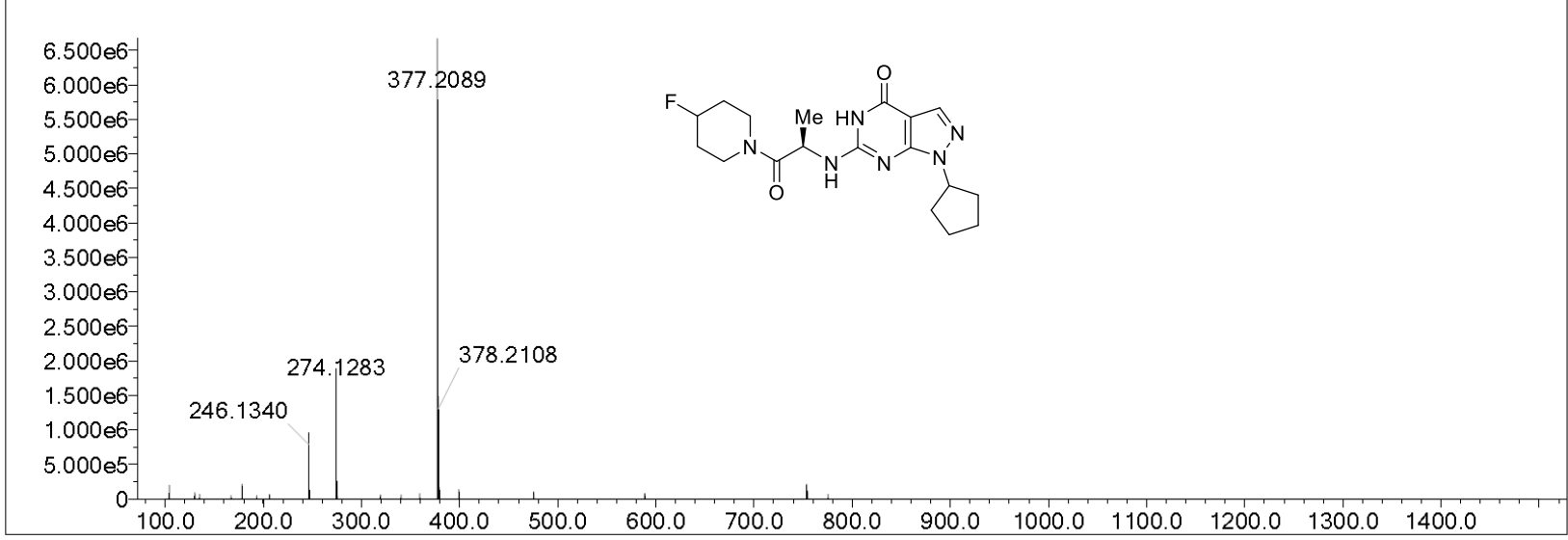

Measured region for $377.2089 \mathrm{~m} / \mathrm{z}$

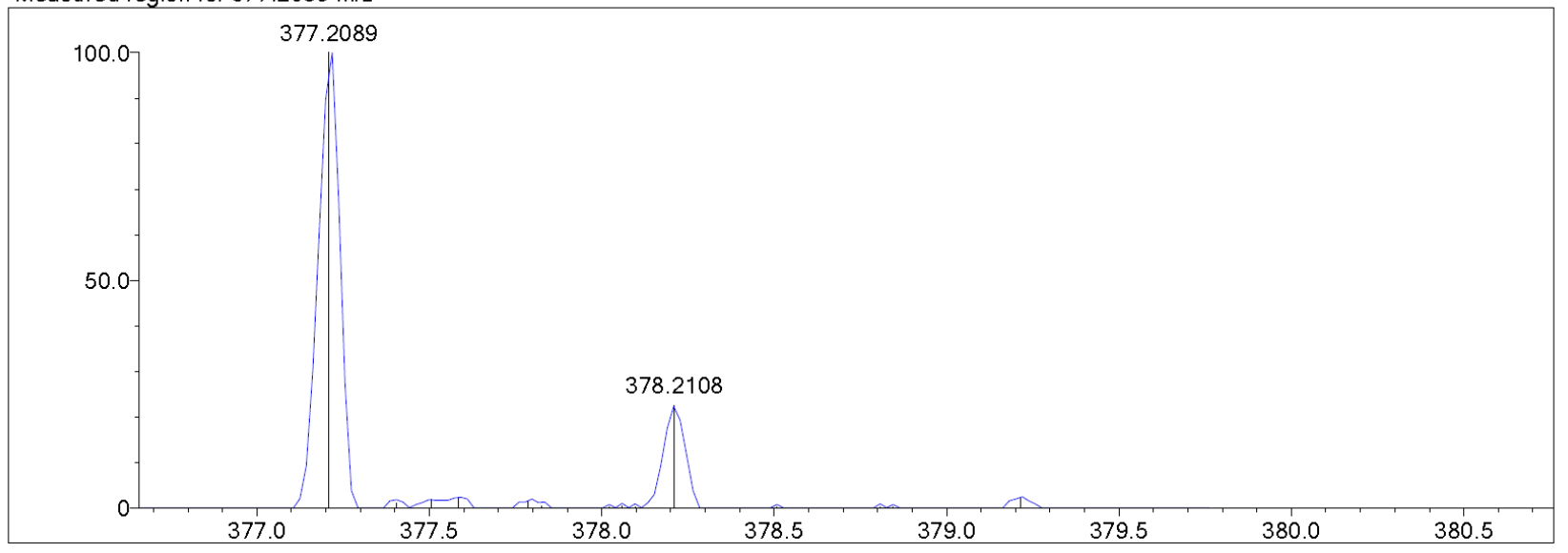

$\mathrm{C} 18 \mathrm{H} 25 \mathrm{~N} 6 \mathrm{O} 2 \mathrm{~F}[\mathrm{M}+\mathrm{H}]+$ : Predicted region for $377.2096 \mathrm{~m} / \mathrm{z}$

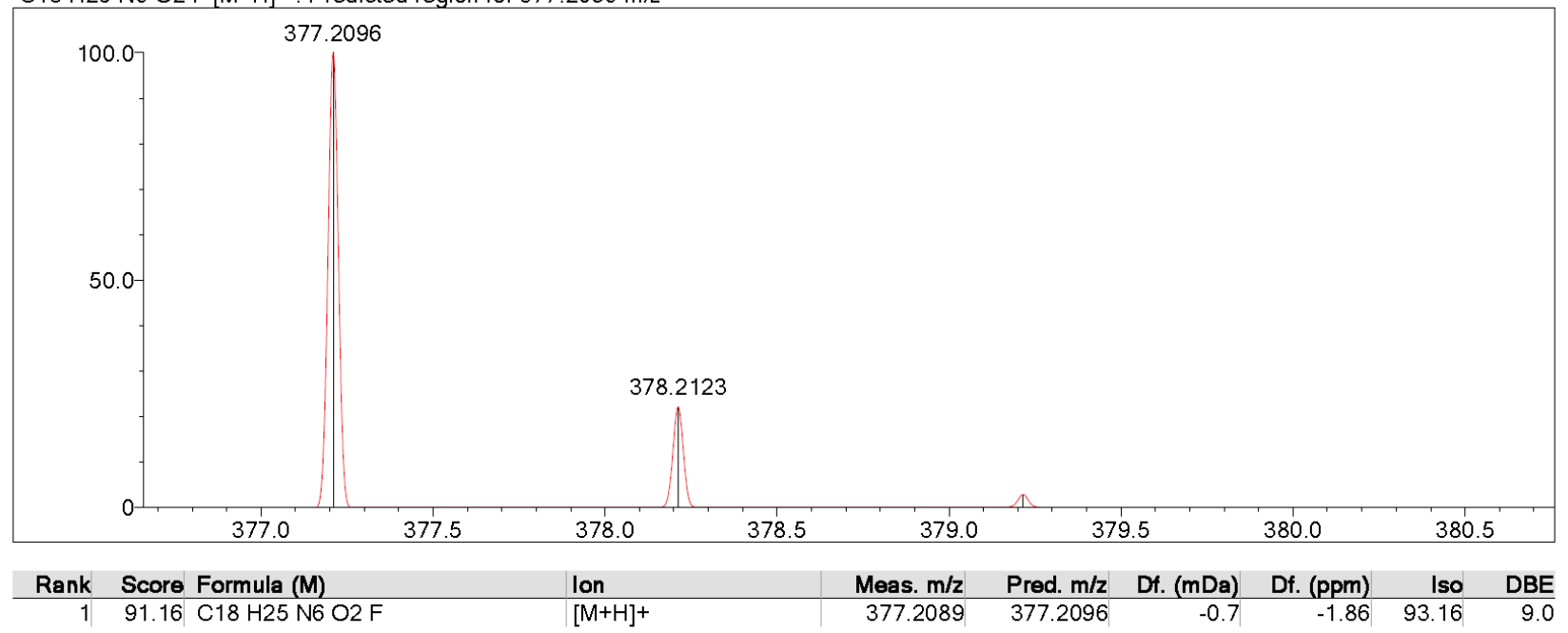




\begin{tabular}{c|c|rr} 
Elmt & Val. & Min & Max \\
\hline $\mathrm{H}$ & 1 & 0 & 30 \\
$\mathrm{~B}$ & 3 & 0 & 0 \\
$\mathrm{C}$ & 4 & 0 & 25
\end{tabular}

Error Margin (ppm): 20 HC Ratio: unlimited

Max Isotopes: all

MSn Iso RI (\%): 75.00
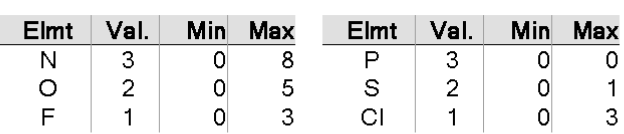

DBE Range: $-1.0-1000.0$

Apply N Rule: no

Isotope RI (\%): 1.00

MSn Logic Mode: AND
Elmt Val. Min Max

\begin{tabular}{l|l|ll}
$\mathrm{Se}$ & 2 & 0 & 0 \\
$\mathrm{Br}$ & 1 & 0 & 0
\end{tabular}

Use Adduct

$\mathrm{Ha}$

Event\#: $1 \mathrm{MS}(\mathrm{E}+)$ Ret. Time : 0.980 Scan\# : 197

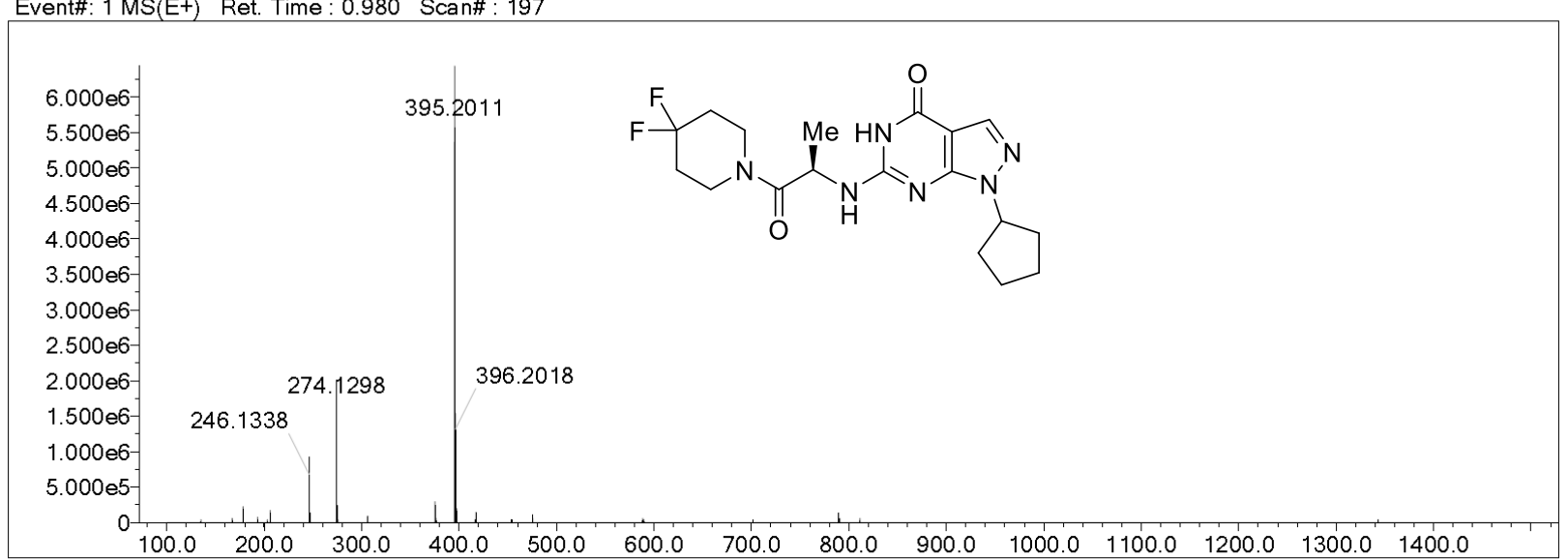

Measured region for $395.2011 \mathrm{~m} / \mathrm{z}$

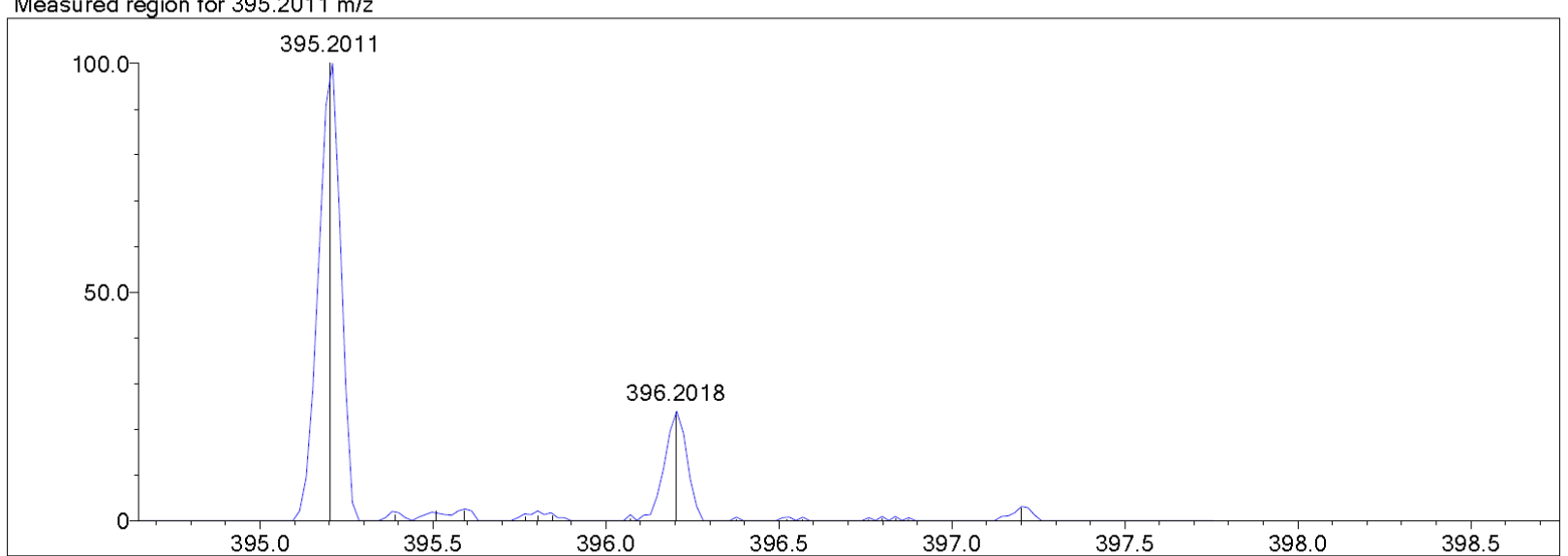

$\mathrm{C} 18 \mathrm{H} 24 \mathrm{~N} 6 \mathrm{O} 2 \mathrm{~F} 2[\mathrm{M}+\mathrm{H}]+$ : Predicted region for $395.2002 \mathrm{~m} / \mathrm{z}$

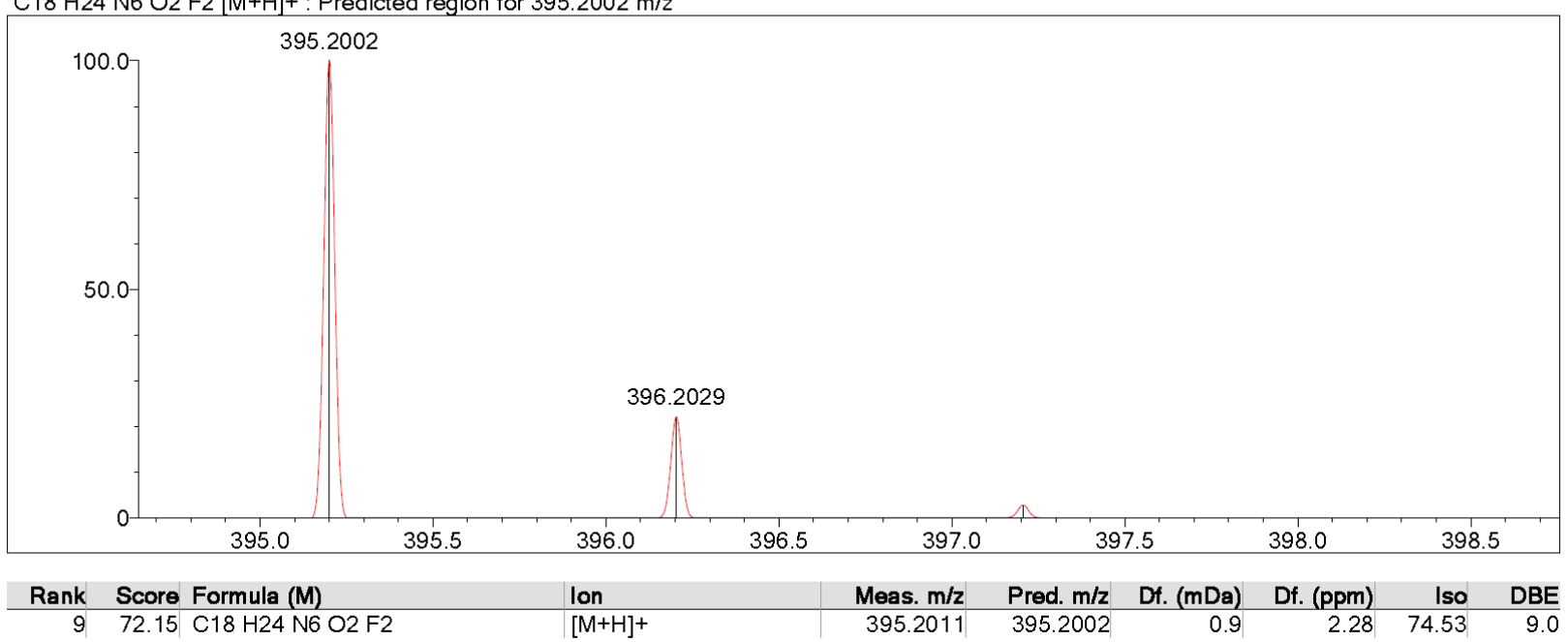




\begin{tabular}{c|c|r|r} 
Elmt & Val. & Min & Max \\
\hline $\mathrm{H}$ & 1 & 0 & 50 \\
$2 \mathrm{H}$ & 1 & 0 & 0 \\
$3 \mathrm{H}$ & 1 & 0 & 0 \\
$\mathrm{~B}$ & 3 & 0 & 0 \\
$\mathrm{C}$ & 4 & 0 & 50 \\
$\mathrm{~N}$ & 3 & 0 & 10
\end{tabular}

\begin{tabular}{c|c|r|r} 
Elmt & Val. & Min & Max \\
\hline $\mathrm{O}$ & 2 & 0 & 8 \\
180 & 2 & 0 & 0 \\
$\mathrm{~F}$ & 1 & 0 & 0 \\
$\mathrm{Si}$ & 4 & 0 & 0 \\
$\mathrm{P}$ & 3 & 0 & 0 \\
$\mathrm{~S}$ & 2 & 0 & 0
\end{tabular}

\begin{tabular}{c|c|rr} 
Elmt & Val. & Min & Max \\
\hline $\mathrm{Cl}$ & 1 & 0 & 0 \\
$\mathrm{~K}$ & 1 & 0 & 0 \\
$\mathrm{Mn}$ & 3 & 0 & 0 \\
$\mathrm{Fe}$ & 2 & 0 & 0 \\
$\mathrm{Cu}$ & 2 & 0 & 0 \\
$\mathrm{Se}$ & 2 & 0 & 0
\end{tabular}

Error Margin (ppm): 20 HC Ratio: 0.0 - 100.0

DBE Range: $-100.0-200.0$

Apply N Rule: no

Isotope RI (\%): 1.00

Max Isotopes: all

MSn Iso RI (\%): 75.00

MSn Logic Mode: AND

\begin{tabular}{c|c|r|r} 
Elmt & Val. & Min & Max \\
\hline $\mathrm{Br}$ & 1 & 0 & 0 \\
$\mathrm{Pd}$ & 2 & 0 & 0 \\
$\mathrm{Te}$ & 2 & 0 & 0 \\
$\mathrm{I}$ & 3 & 0 & 0 \\
$\mathrm{Pt}$ & 2 & 0 & 0 \\
$\mathrm{Au}$ & 1 & 0 & 0
\end{tabular}

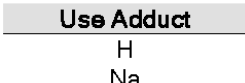

Electron lons: both

Use MSn Info: no

Isotope Res: 10000

Max Results: 10000

Event\#: $1 \mathrm{MS}(\mathrm{E}+)$ Ret. Time : 0.987 Scan\# : 149

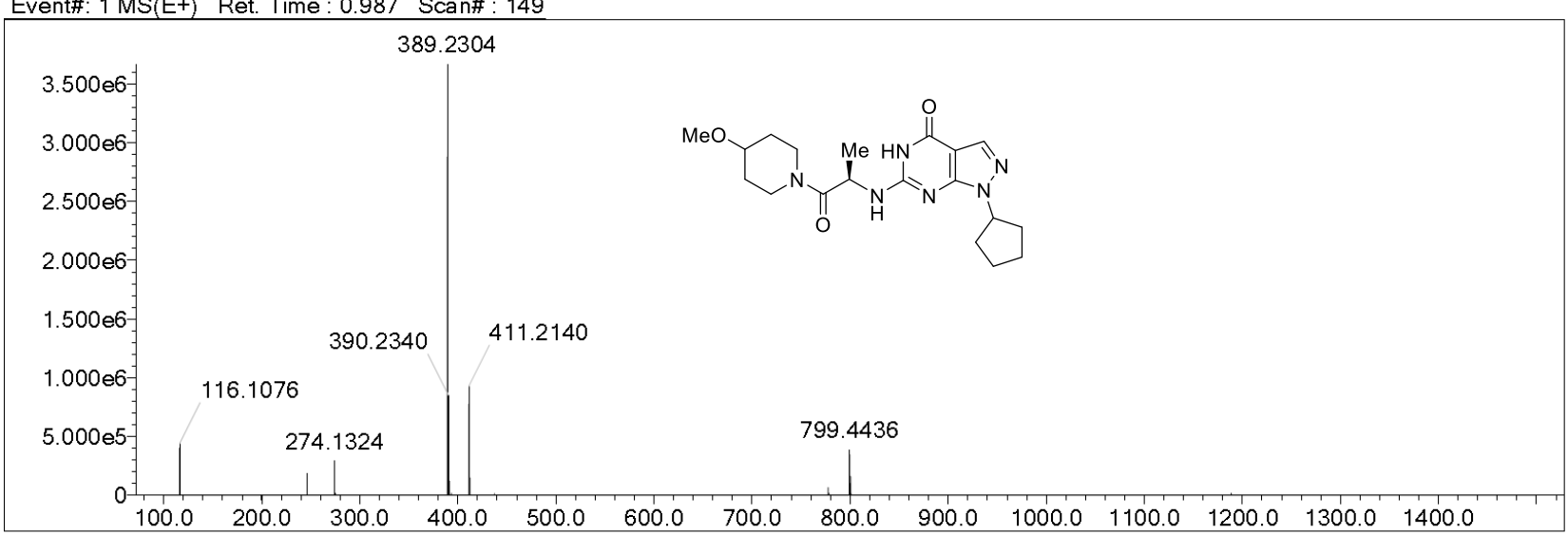

Measured region for $389.2304 \mathrm{~m} / \mathrm{z}$

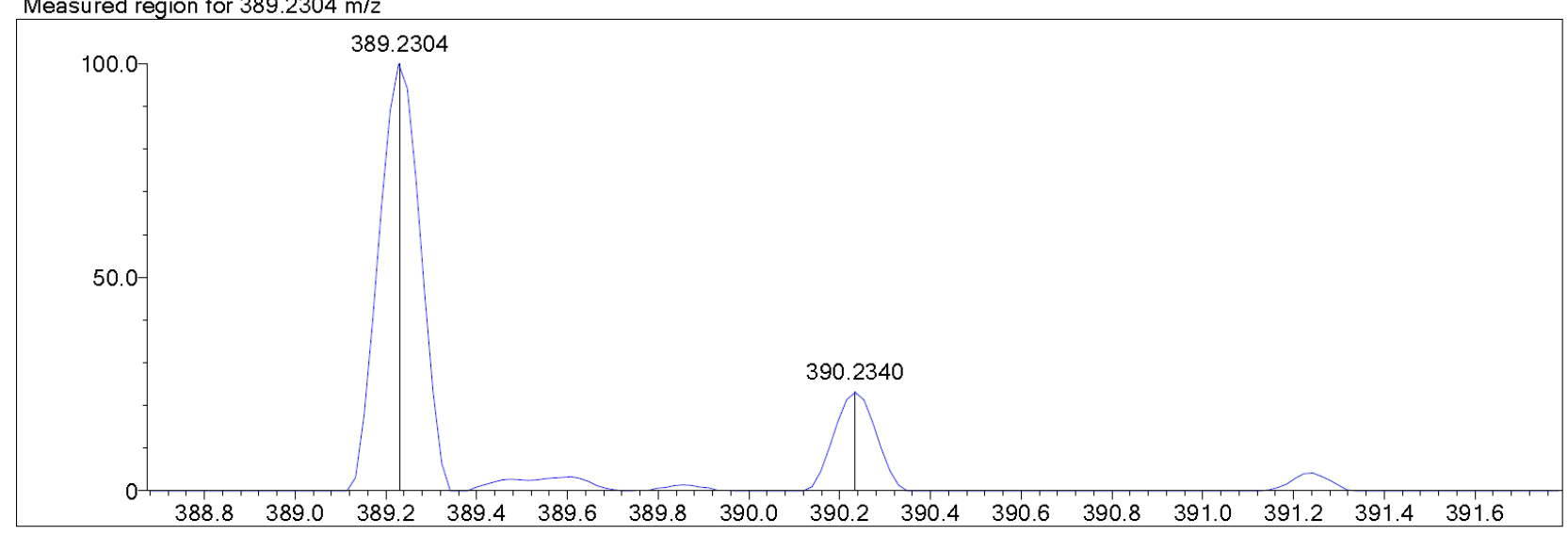

$\mathrm{C} 19 \mathrm{H} 28 \mathrm{~N} 6 \mathrm{O} 3[\mathrm{M}+\mathrm{H}]+$ : Predicted region for $389.2296 \mathrm{~m} / \mathrm{z}$

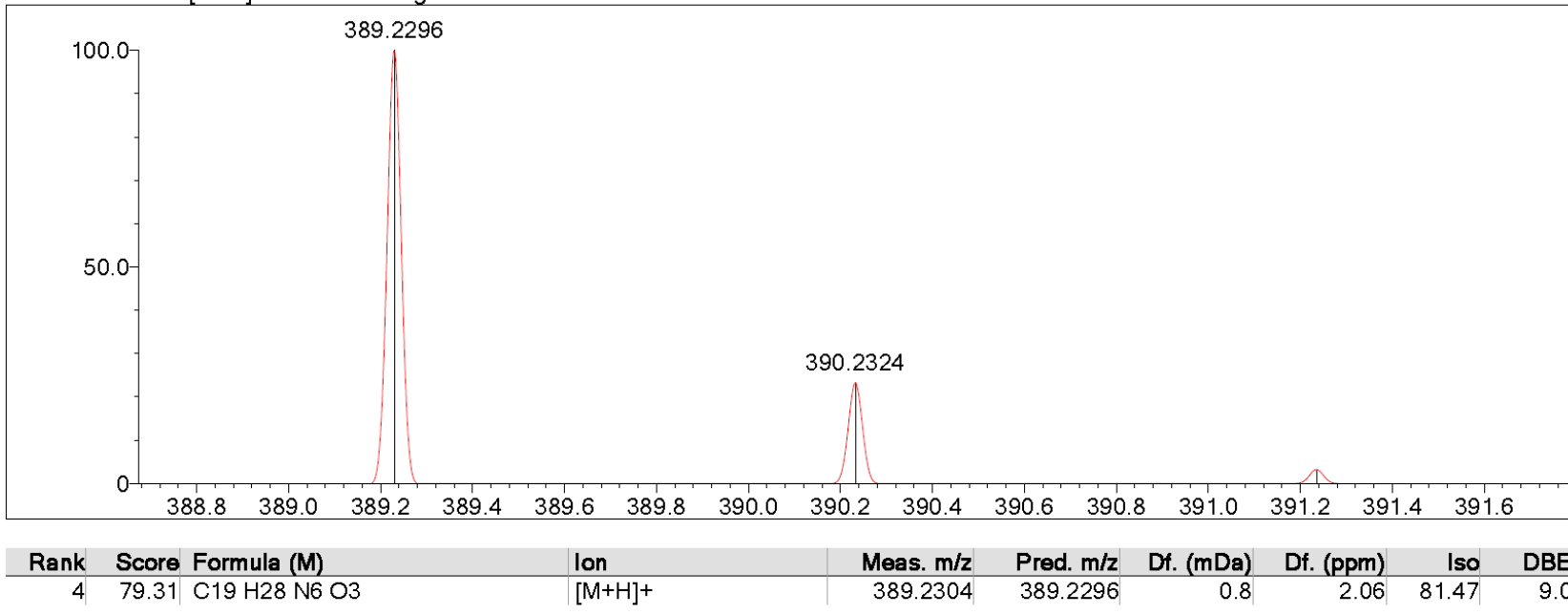




\begin{tabular}{c|c|r|r} 
Elmt & Val. & Min & Max \\
\hline $\mathrm{H}$ & 1 & 12 & 38 \\
$2 \mathrm{H}$ & 1 & 0 & 0 \\
$\mathrm{~B}$ & 3 & 0 & 0 \\
$\mathrm{C}$ & 4 & 12 & 20
\end{tabular}

Error Margin (ppm): 30

HC Ratio: unlimited

Max Isotopes: all

MSn Iso RI (\%): 75.00

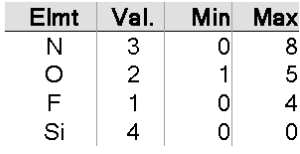

DBE Range: $-1.0-1000.0$

Apply N Rule: no

Isotope RI (\%): 1.00

MSn Logic Mode: AND

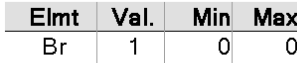

Use Adduct $\mathrm{H}$

$\mathrm{Na}$

Cl

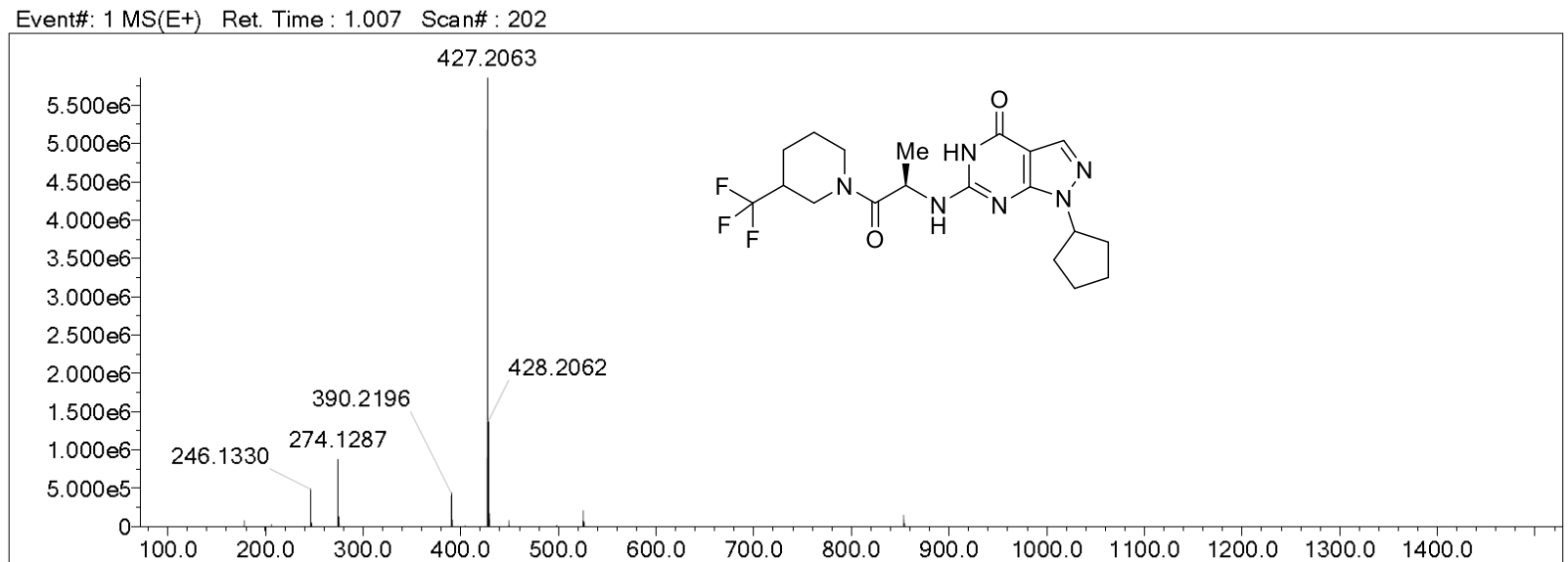

Measured region for $427.2063 \mathrm{~m} / \mathrm{z}$

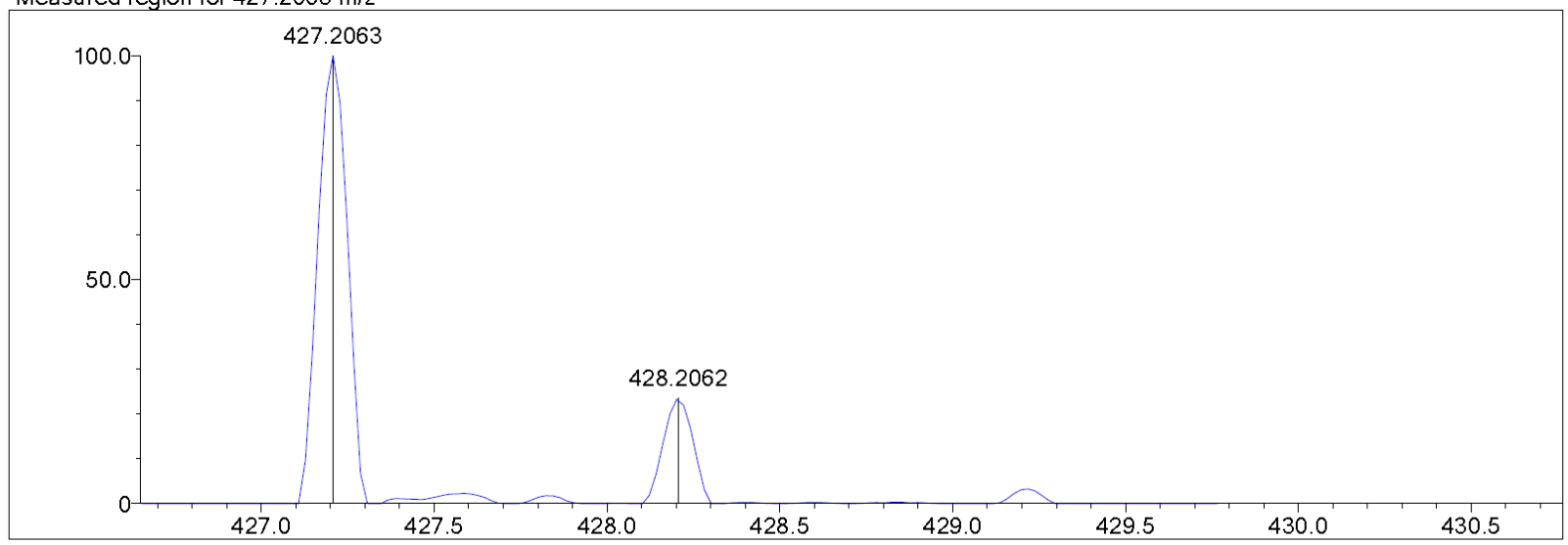

$\mathrm{C} 19 \mathrm{H} 25 \mathrm{~N} 6 \mathrm{O} 2 \mathrm{~F} 3[\mathrm{M}+\mathrm{H}]+$ : Predicted region for $427.2064 \mathrm{~m} / \mathrm{z}$

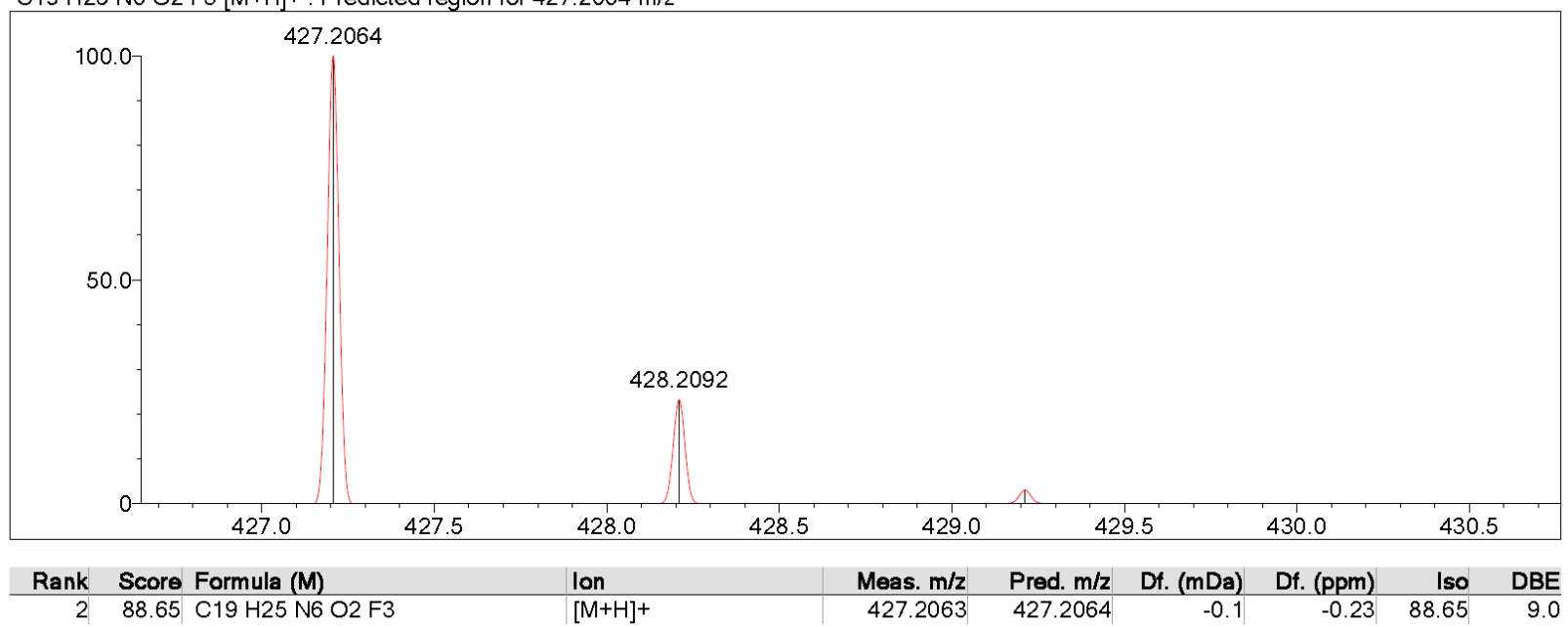




\begin{tabular}{c|c|rr} 
Elnt & Val. & Min & Max \\
\hline $\mathrm{H}$ & 1 & 15 & 25 \\
$2 \mathrm{H}$ & 1 & 0 & 0 \\
$\mathrm{~B}$ & 3 & 0 & 0 \\
$\mathrm{C}$ & 4 & 18 & 20 \\
$\mathrm{~N}$ & 3 & 2 & 7 \\
$\mathrm{O}$ & 2 & 1 & 2
\end{tabular}

Error Margin (ppm): 200 HC Ratio: unlimited

Max Isotopes: all

MSn Iso RI (\%): 75.00

\begin{tabular}{c|c|r|r} 
Elmt & Vol. & Mln & Max \\
\hline F & 1 & 1 & 1 \\
Si & 4 & 0 & 0 \\
P & 3 & 0 & 0 \\
S & 2 & 0 & 0 \\
CI & 1 & 0 & 0 \\
K & 1 & 0 & 0
\end{tabular}

DBE Range: not fixed Apply N Rule: yes Isotope RI (\%): 1.00

MSn Logic Mode: AND

\begin{tabular}{c|c|r|r} 
Elmt & Val. & Min & Max \\
\hline $\mathrm{Se}$ & 2 & 0 & 0 \\
$\mathrm{Br}$ & 1 & 0 & 0 \\
$\mathrm{Pd}$ & 2 & 0 & 0 \\
$\mathrm{I}$ & 3 & 0 & 0 \\
$\mathrm{Pt}$ & 2 & 0 & 0
\end{tabular}

Electron lons: both Use MSn Info: no

Isotope Res: 10000

Max Results: 50

Event\#: $1 \mathrm{MS}(\mathrm{E}+)$ Ret. Time : 0.547 Scan\# : 83

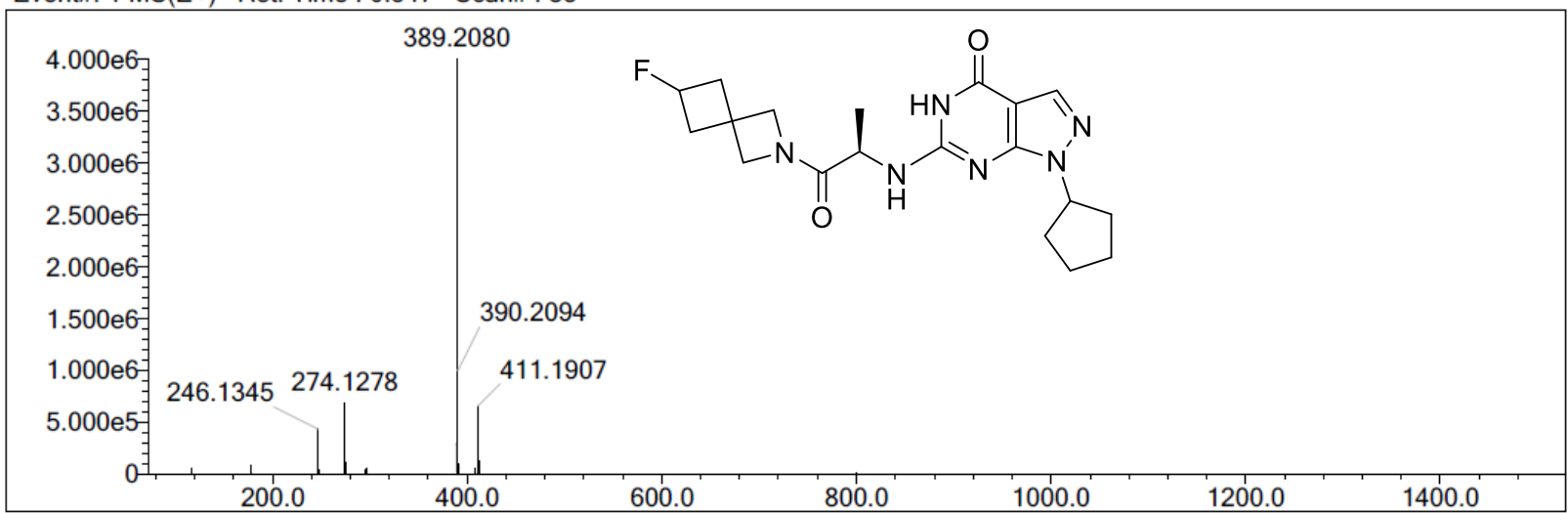

Measured region for $389.2080 \mathrm{~m} / \mathrm{z}$

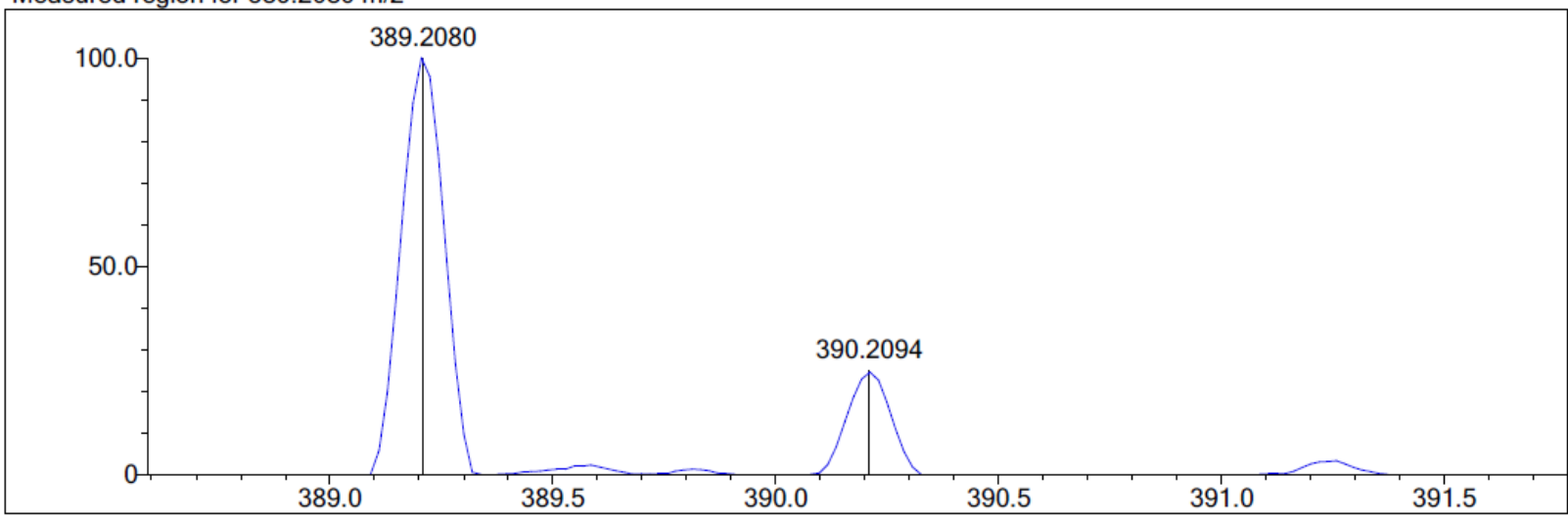

C19 H25 N6 O2 F [M+H]+ : Predicted region for $389.2096 \mathrm{~m} / \mathrm{z}$

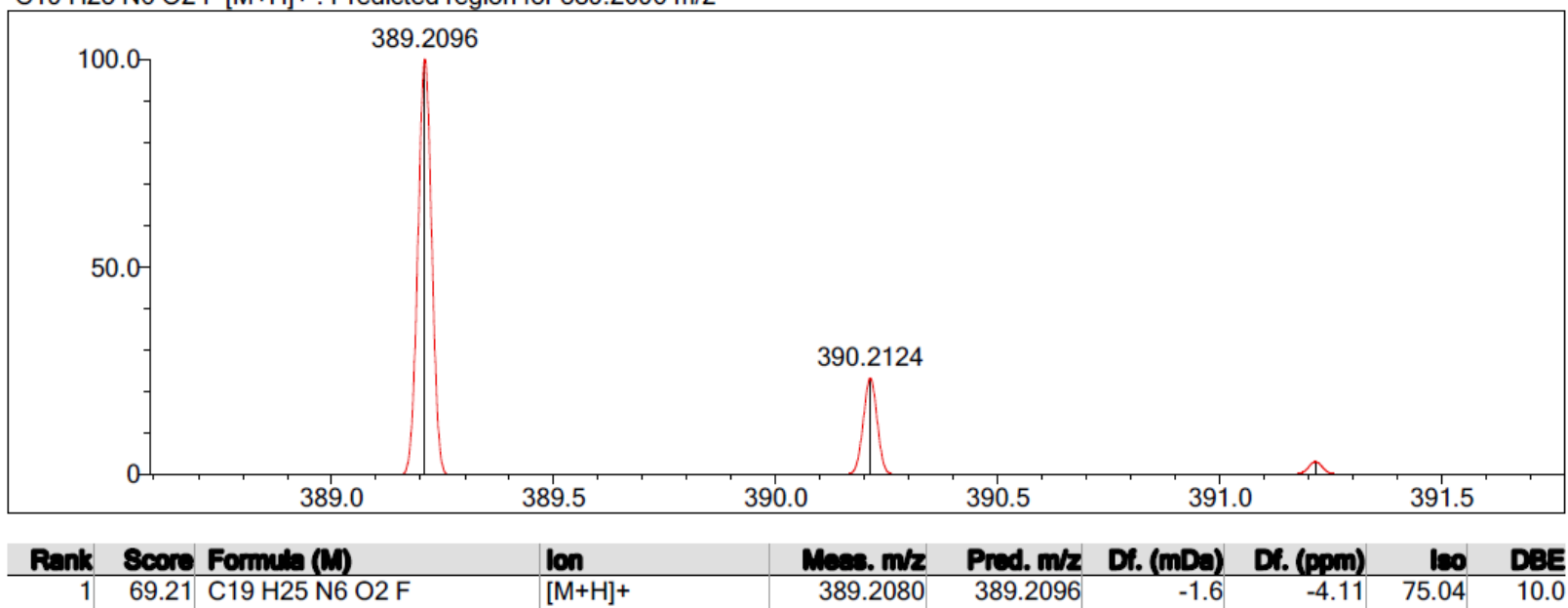




\begin{tabular}{c|c|rr} 
Enint & Val. & Min & Max \\
\hline $\mathrm{H}$ & 1 & 15 & 25 \\
$2 \mathrm{H}$ & 1 & 0 & 0 \\
$\mathrm{~B}$ & 3 & 0 & 0 \\
$\mathrm{C}$ & 4 & 18 & 20 \\
$\mathrm{~N}$ & 3 & 2 & 7 \\
$\mathrm{O}$ & 2 & 1 & 2
\end{tabular}

Error Margin (ppm): 200 HC Ratio: unlimited

Max Isotopes: all MSn Iso RI (\%): 75.00

\begin{tabular}{c|c|r|r} 
Elmt & Val. & Mln & Max \\
\hline $\mathrm{F}$ & 1 & 0 & 0 \\
$\mathrm{Si}$ & 4 & 0 & 0 \\
$\mathrm{P}$ & 3 & 0 & 0 \\
$\mathrm{~S}$ & 2 & 0 & 0 \\
$\mathrm{Cl}$ & 1 & 1 & 1 \\
$\mathrm{~K}$ & 1 & 0 & 0
\end{tabular}

DBE Range: not fixed

Apply N Rule: yes

Isotope RI (\%): 1.00

MSn Logic Mode: AND

\section{Une Adduct}

$\mathrm{H}$

$\mathrm{Na}$

$\mathrm{NH} 4$

$\mathrm{K}$

Event\#: $1 \mathrm{MS}\left(\mathrm{E}^{+}\right)$Ret. Time : 0.560 Scan\# : 85

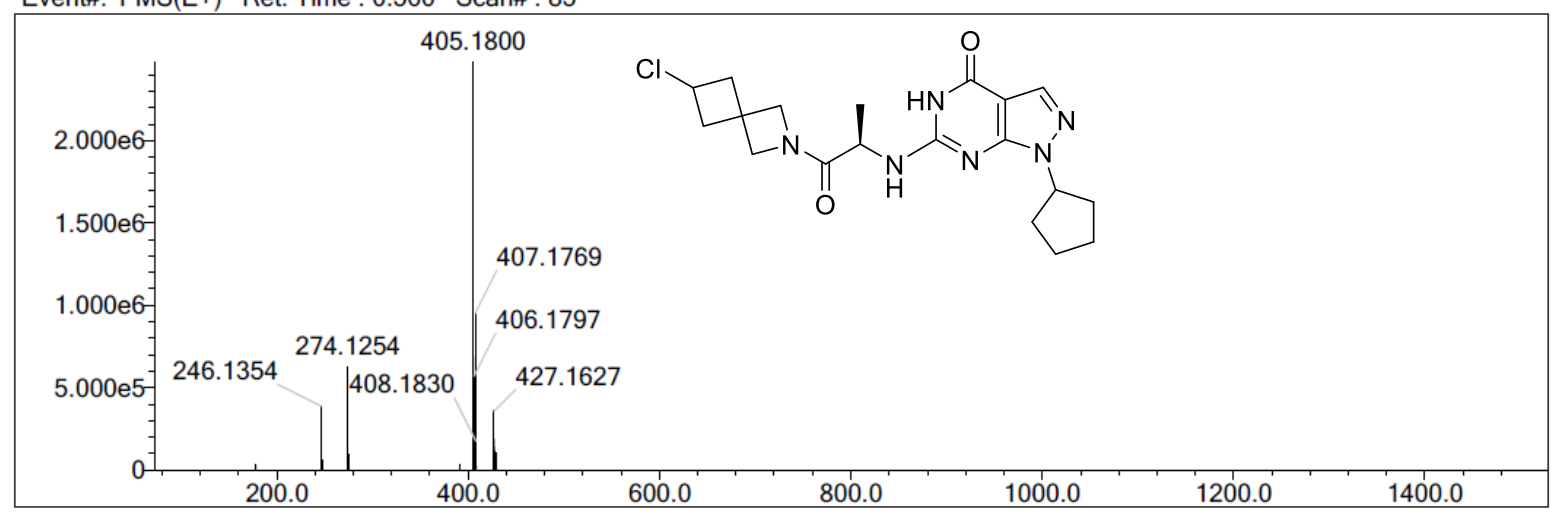

Measured region for $405.1800 \mathrm{~m} / \mathrm{z}$

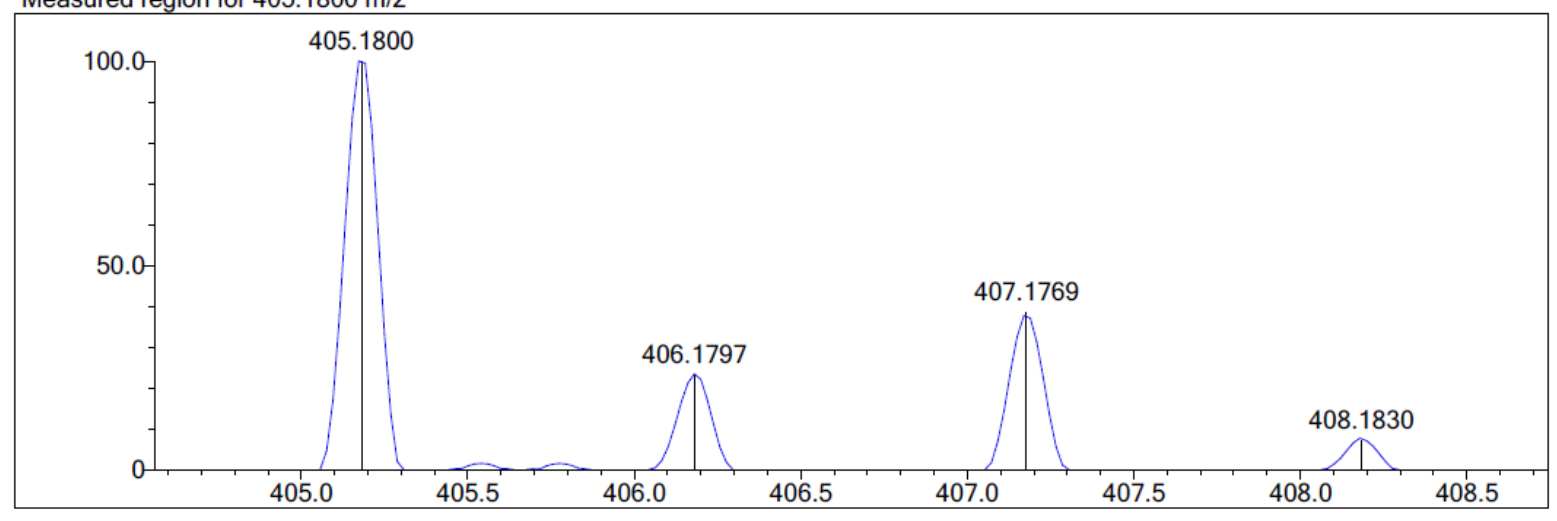

$\mathrm{C} 19 \mathrm{H} 25 \mathrm{~N} 6 \mathrm{O} 2 \mathrm{Cl}[\mathrm{M}+\mathrm{H}]+$ : Predicted region for $405.1800 \mathrm{~m} / \mathrm{z}$

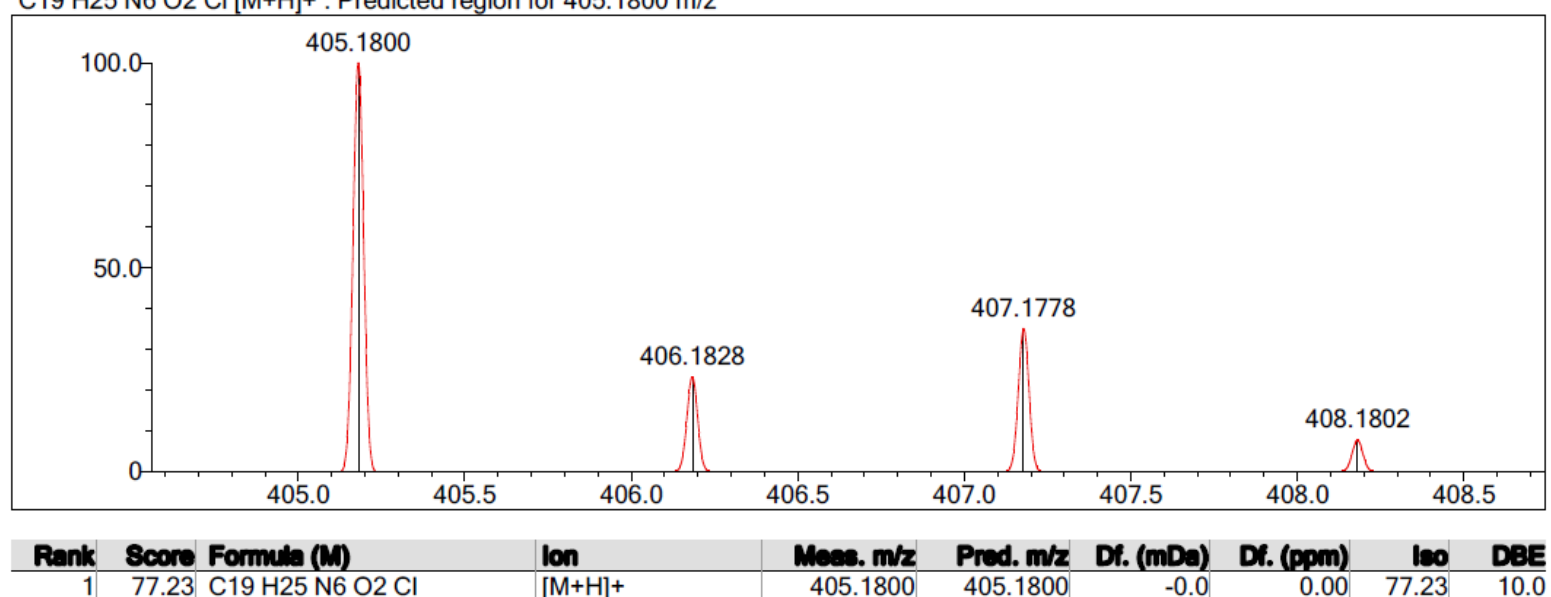




\section{HPLC spectrum for the purity of representative target compounds.}

Compound $1 \mathbf{c}$ were eluted with a solution of $\mathrm{MeOH}$ in water $(50 \%, \mathrm{v} / \mathrm{v})$ at a temperature of $25^{\circ} \mathrm{C}$, and a flow rate of $1.0 \mathrm{~mL} / \mathrm{min}$.

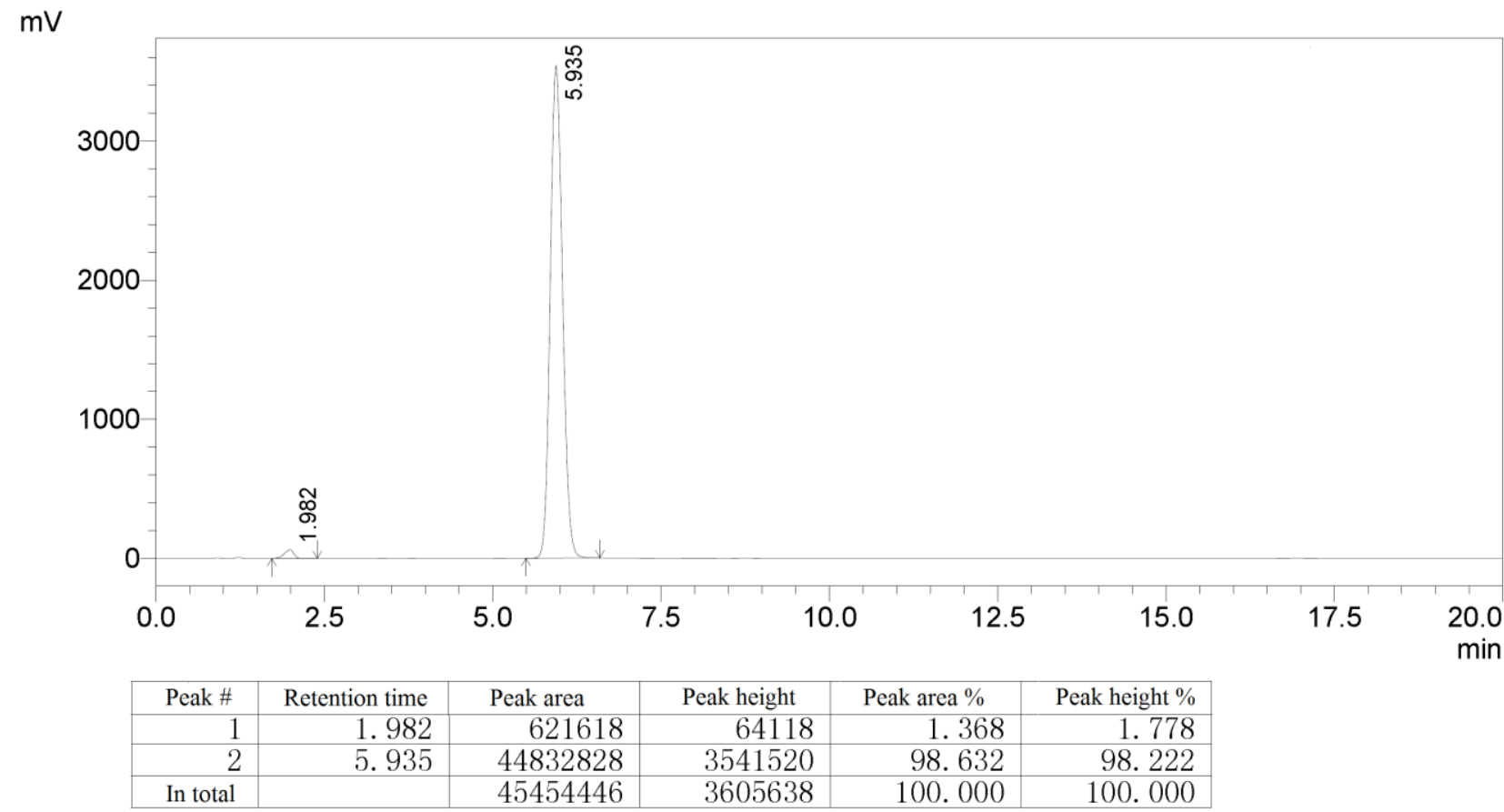

Compound $2 \mathbf{a}$ were eluted with a solution of $\mathrm{MeOH}$ in water $(50 \%, \mathrm{v} / \mathrm{v})$ at a temperature of $25^{\circ} \mathrm{C}$, and a flow rate of $1.0 \mathrm{~mL} / \mathrm{min}$.

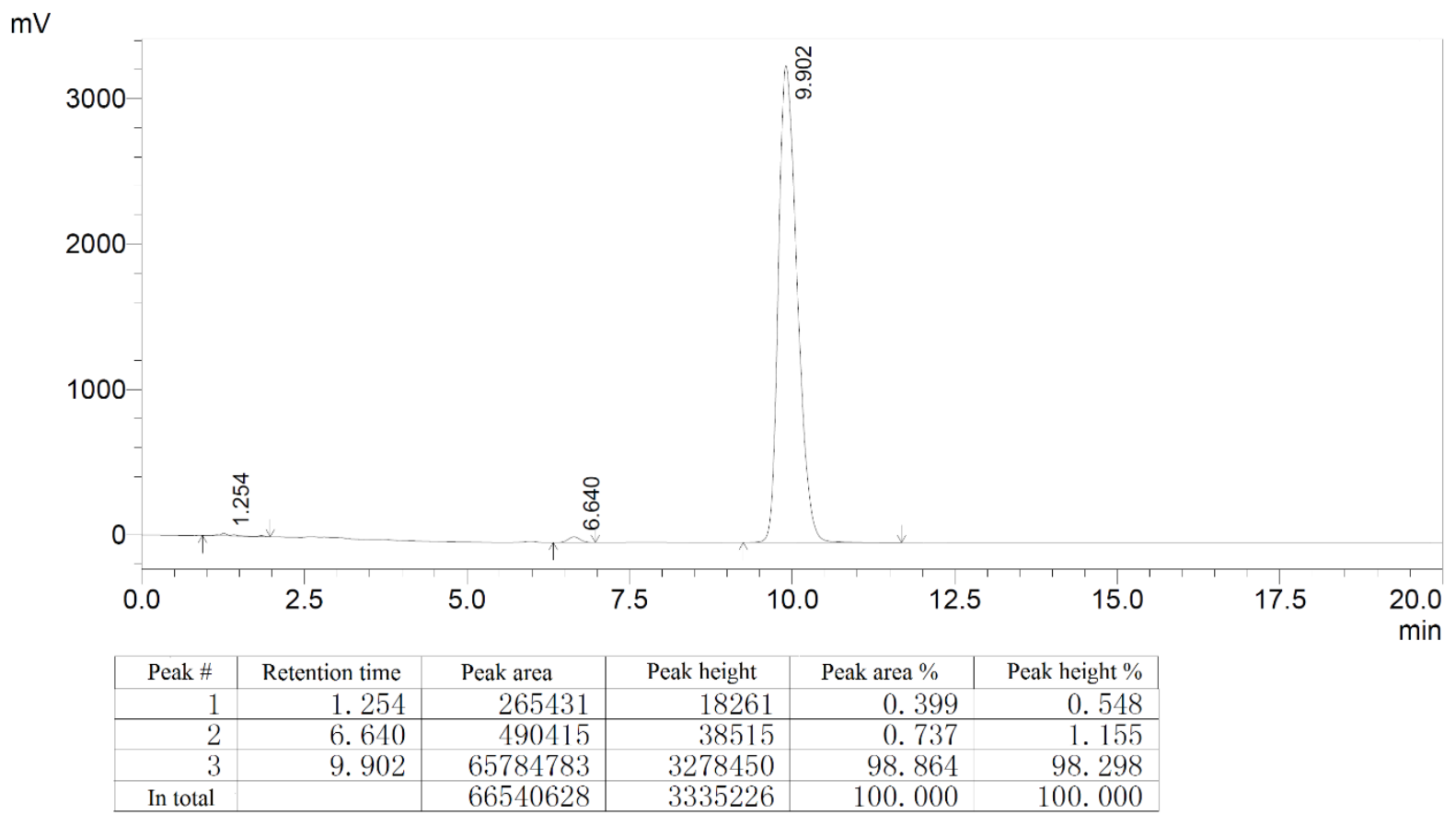


Compound $2 \mathbf{d}$ were eluted with a solution of $\mathrm{MeOH}$ in water $(50 \%, \mathrm{v} / \mathrm{v})$ at a temperature of $25^{\circ} \mathrm{C}$, and a flow rate of $1.0 \mathrm{~mL} / \mathrm{min}$.

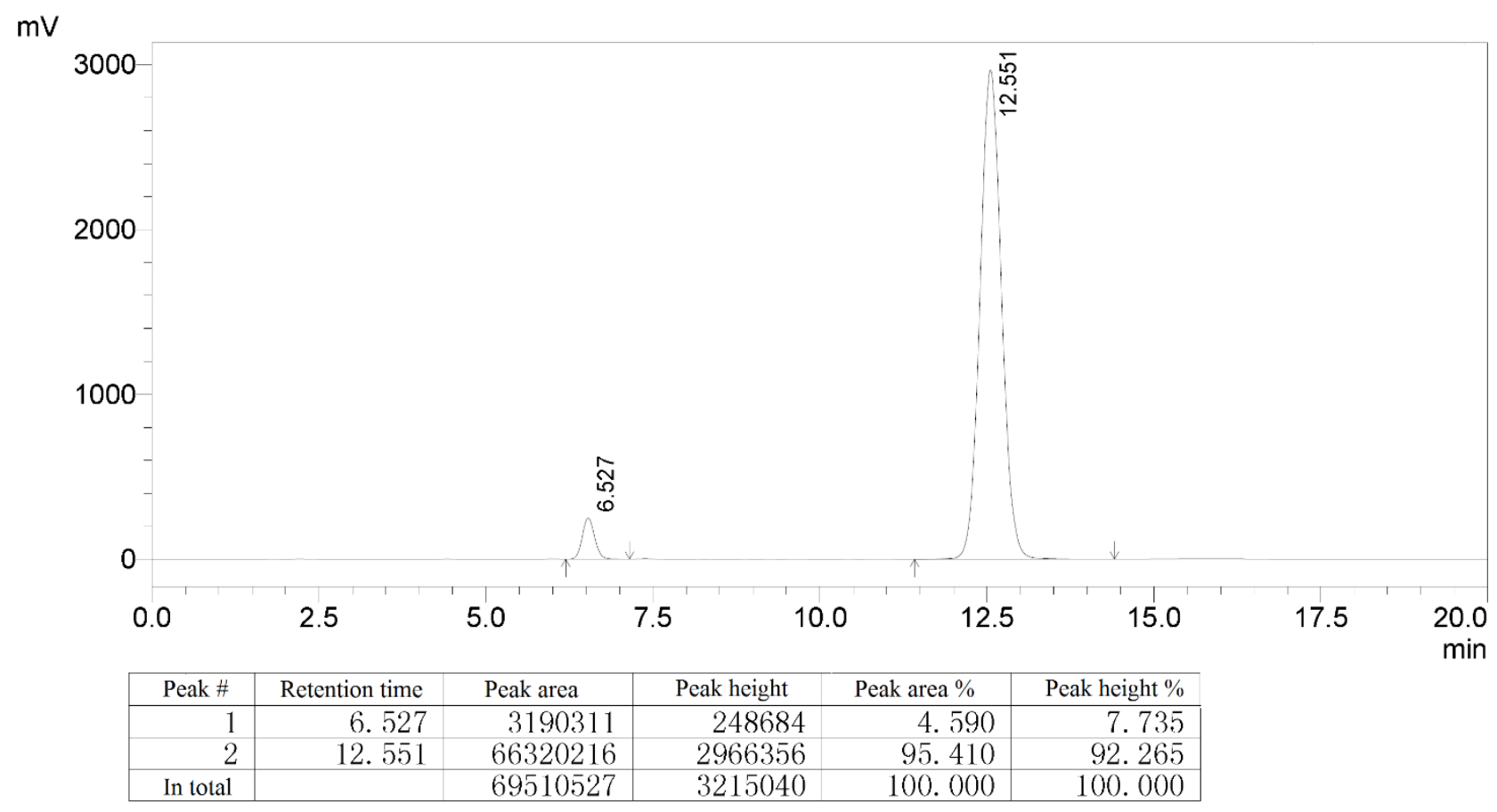

Compound $2 \mathbf{e}$ were eluted with a solution of $\mathrm{MeOH}$ in water $(70 \%, \mathrm{v} / \mathrm{v})$ at a temperature of $25^{\circ} \mathrm{C}$, and a flow rate of $1.0 \mathrm{~mL} / \mathrm{min}$.

$\mathrm{mV}$

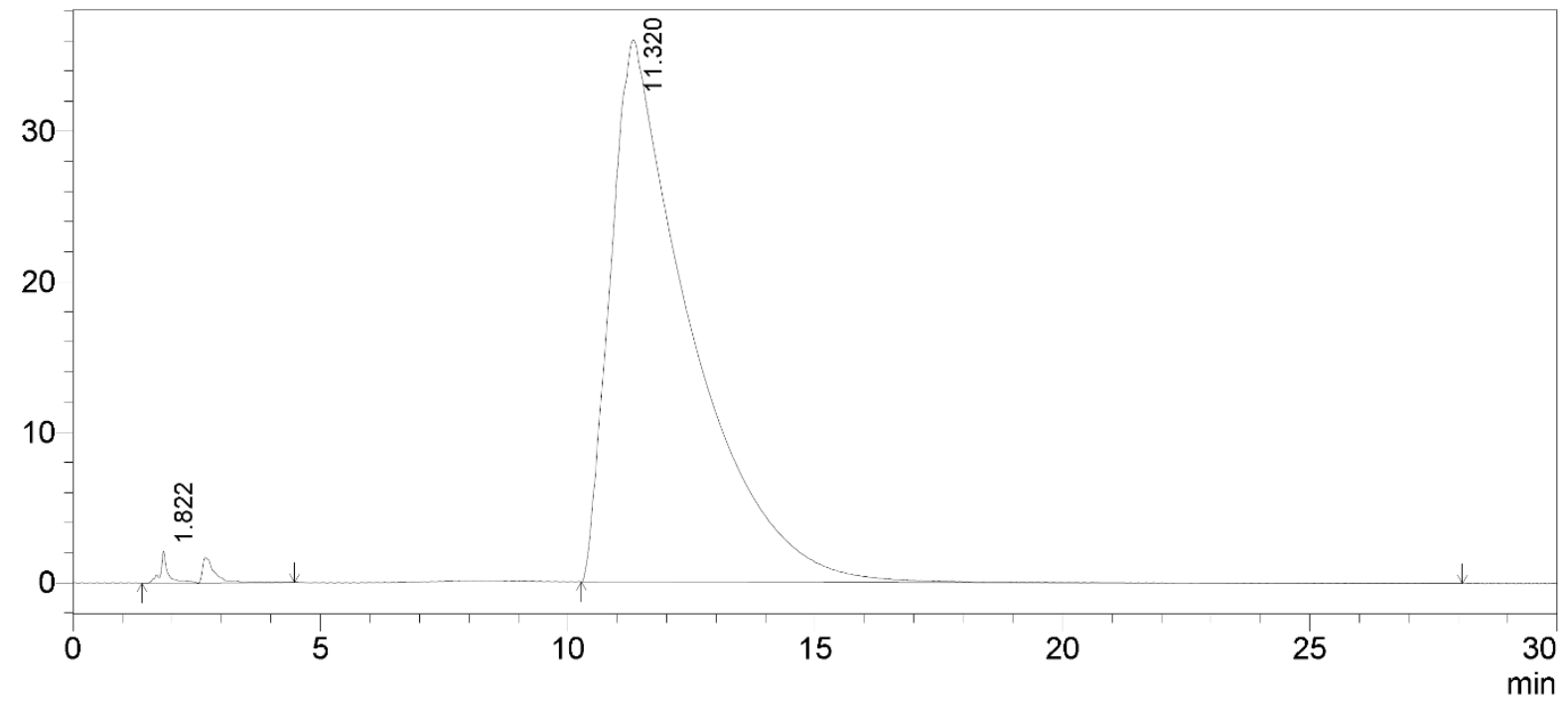

\begin{tabular}{|r|r|r|r|r|r|}
\hline \multicolumn{1}{|c|}{ Peak \# } & Retention time & Peak area & Peak height & Peak area \% & Peak height \% \\
\hline 1 & 1.822 & 52301 & 2134 & 1.276 & 5.596 \\
\hline 2 & 11.320 & 4045486 & 35997 & 98.724 & 94.404 \\
\hline In total & & 4097787 & 38131 & 100.000 & 100.000 \\
\hline
\end{tabular}


Compound $2 \mathrm{~g}$ were eluted with a solution of $\mathrm{MeOH}$ in water $(50 \%, \mathrm{v} / \mathrm{v})$ at a temperature of $25^{\circ} \mathrm{C}$, and a flow rate of $1.0 \mathrm{~mL} / \mathrm{min}$.

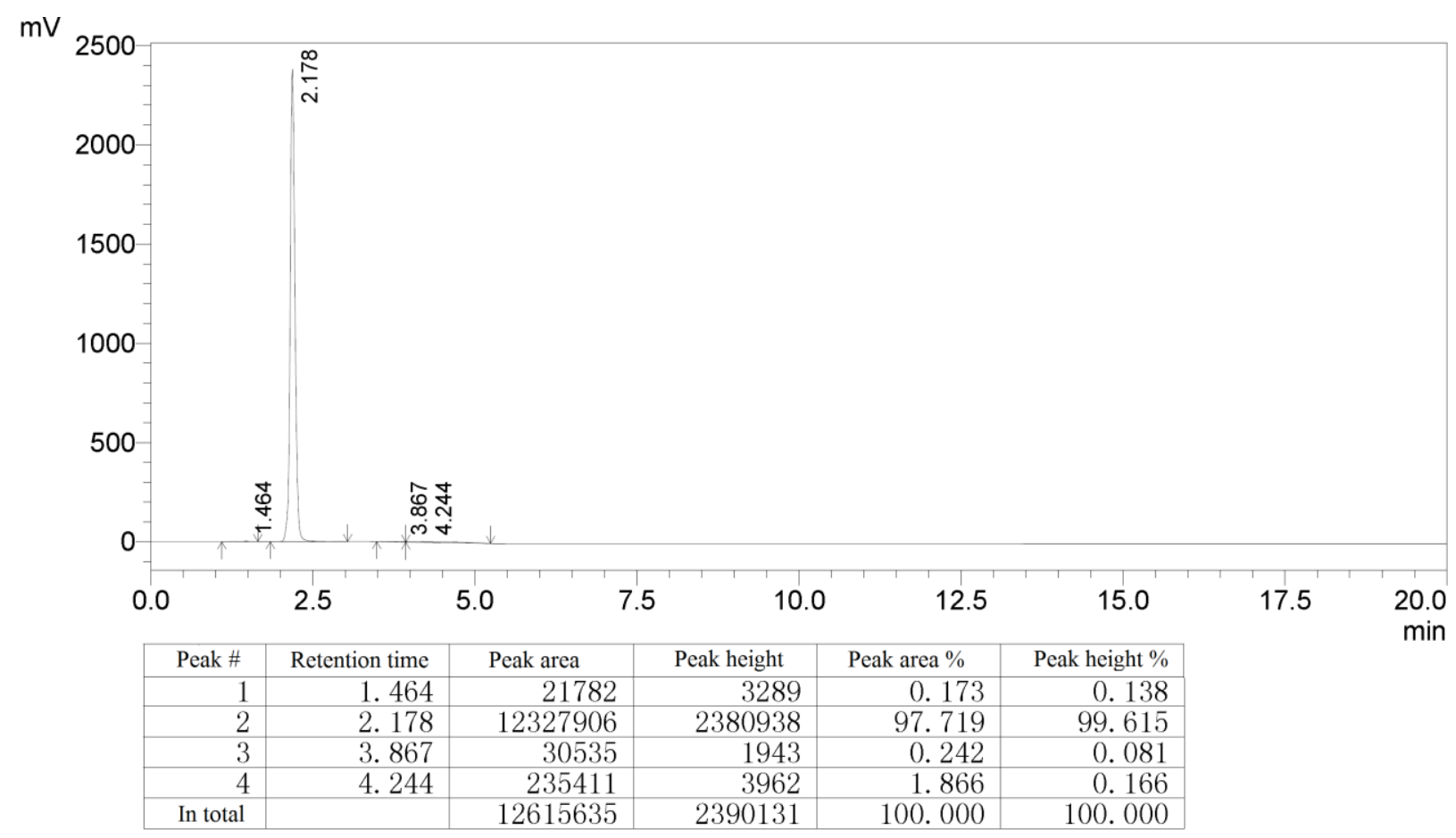

Compound 3a were eluted with a solution of $\mathrm{MeOH}$ in water $(70 \%, \mathrm{v} / \mathrm{v})$ at a temperature of $25^{\circ} \mathrm{C}$, and a flow rate of $1.0 \mathrm{~mL} / \mathrm{min}$.

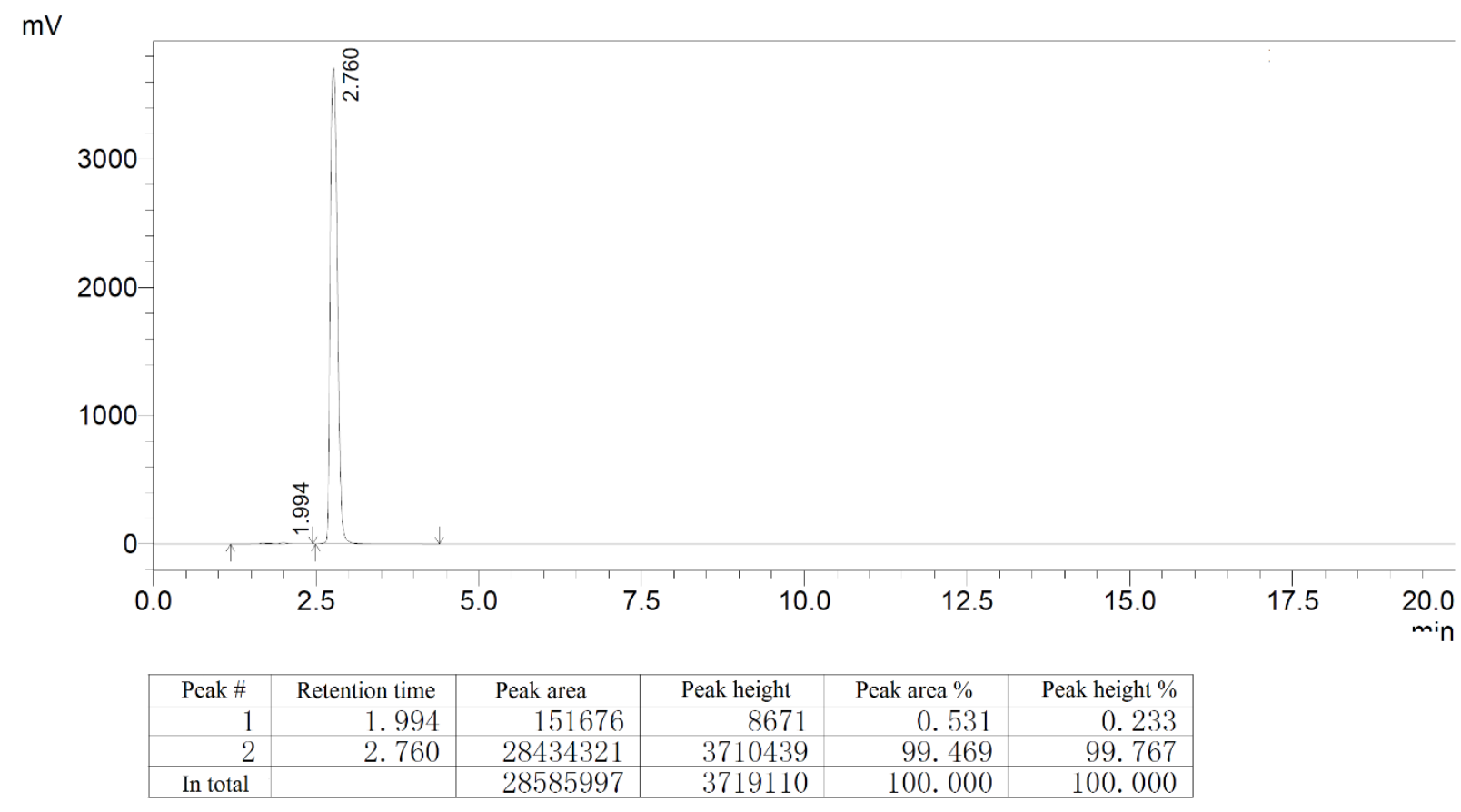


Compound $\mathbf{3 b}$ were eluted with a solution of $\mathrm{MeOH}$ in water $(50 \%, \mathrm{v} / \mathrm{v})$ at a temperature of $25^{\circ} \mathrm{C}$, and a flow rate of $1.0 \mathrm{~mL} / \mathrm{min}$.

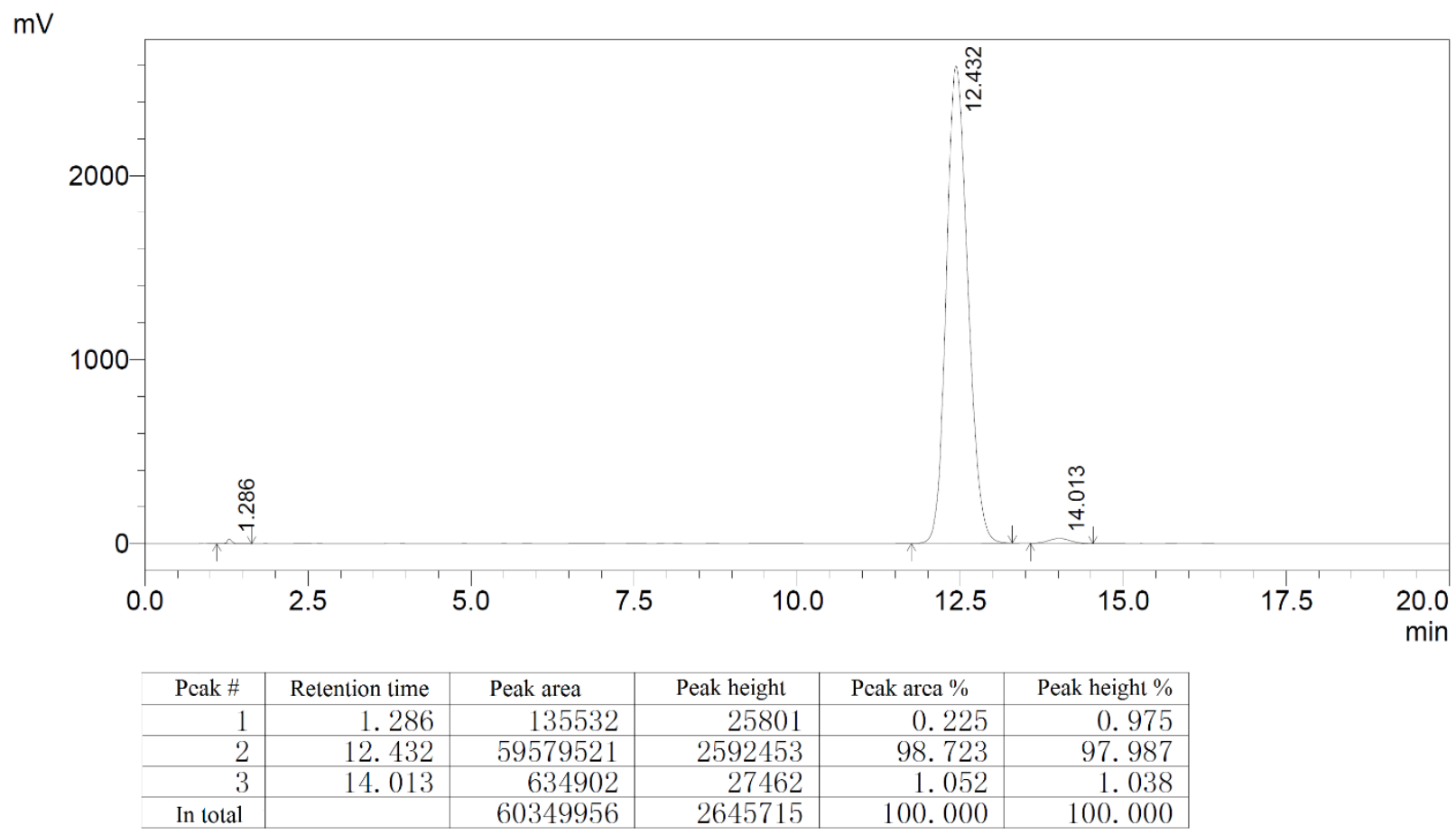

Compound $3 \mathbf{c}$ were eluted with a solution of $\mathrm{MeOH}$ in water $(50 \%, \mathrm{v} / \mathrm{v})$ at a temperature of $25^{\circ} \mathrm{C}$, and a flow rate of $1.0 \mathrm{~mL} / \mathrm{min}$.

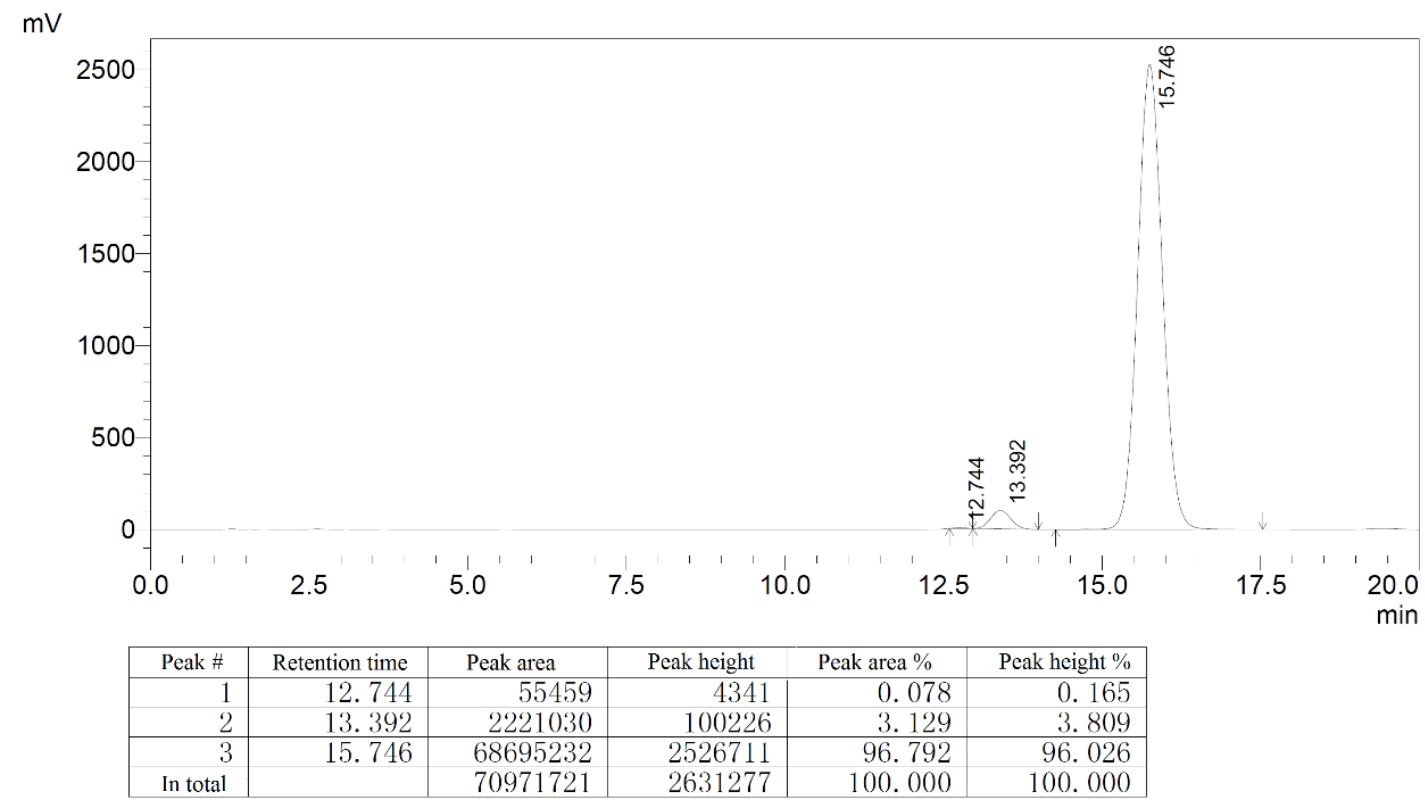


Compound $3 \mathbf{e}$ were eluted with a solution of $\mathrm{MeOH}$ in water $(50 \%, \mathrm{v} / \mathrm{v})$ at a temperature of $25^{\circ} \mathrm{C}$, and a flow rate of $1.0 \mathrm{~mL} / \mathrm{min}$.

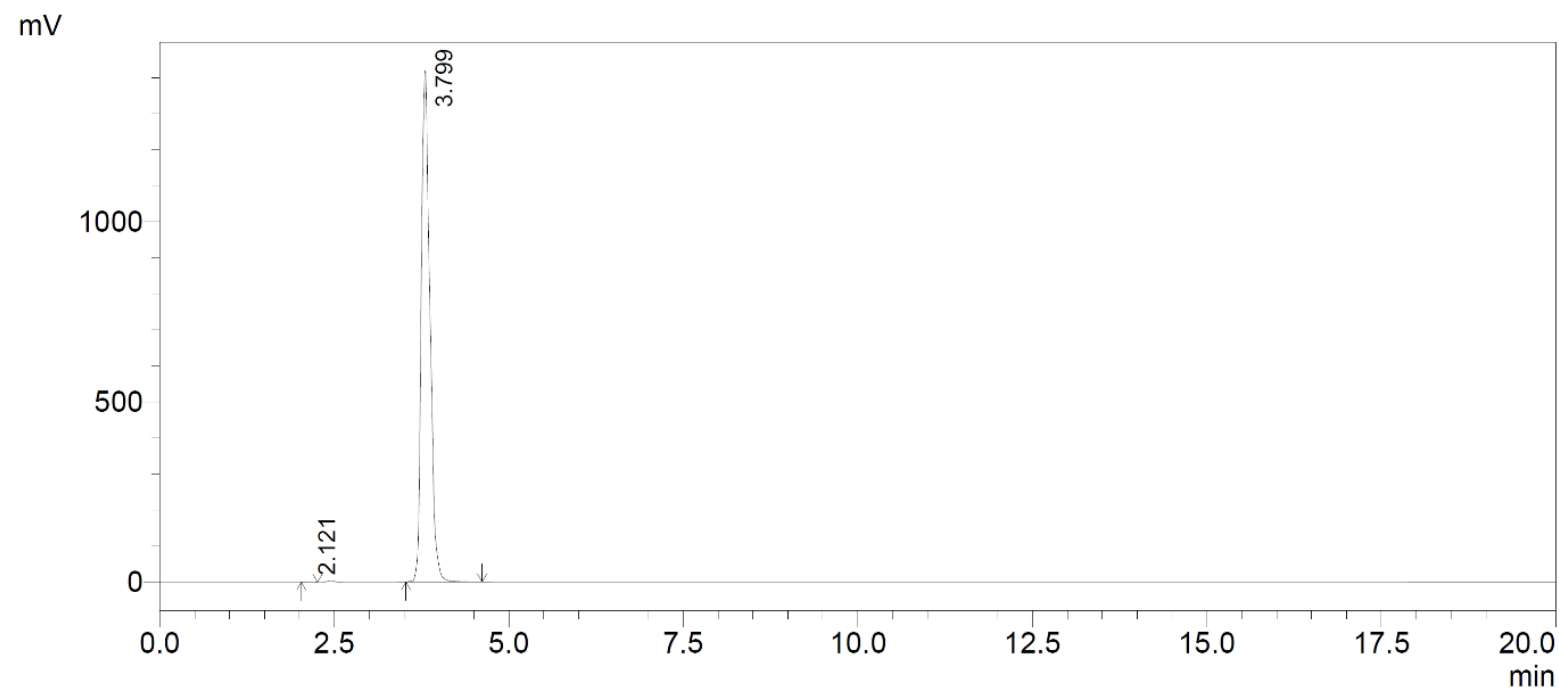

\begin{tabular}{|r|r|r|r|r|r|}
\hline \multicolumn{1}{|c|}{ Peak \# } & Retention time & Peak area & Peak height & Peak area \% & Peak height \% \\
\hline 1 & 2.121 & 5942 & 1197 & 0.048 & 0.084 \\
2 & 3.799 & 12265891 & 1418690 & 99.952 & 99.916 \\
In total & & 12271833 & 1419887 & 100.000 & 100.000
\end{tabular}

Compound $4 \mathbf{a}$ were eluted with a solution of $\mathrm{MeOH}$ in water $(50 \%, \mathrm{v} / \mathrm{v})$ at a temperature of $25^{\circ} \mathrm{C}$, and a flow rate of $1.0 \mathrm{~mL} / \mathrm{min}$.

$\mathrm{mV}$

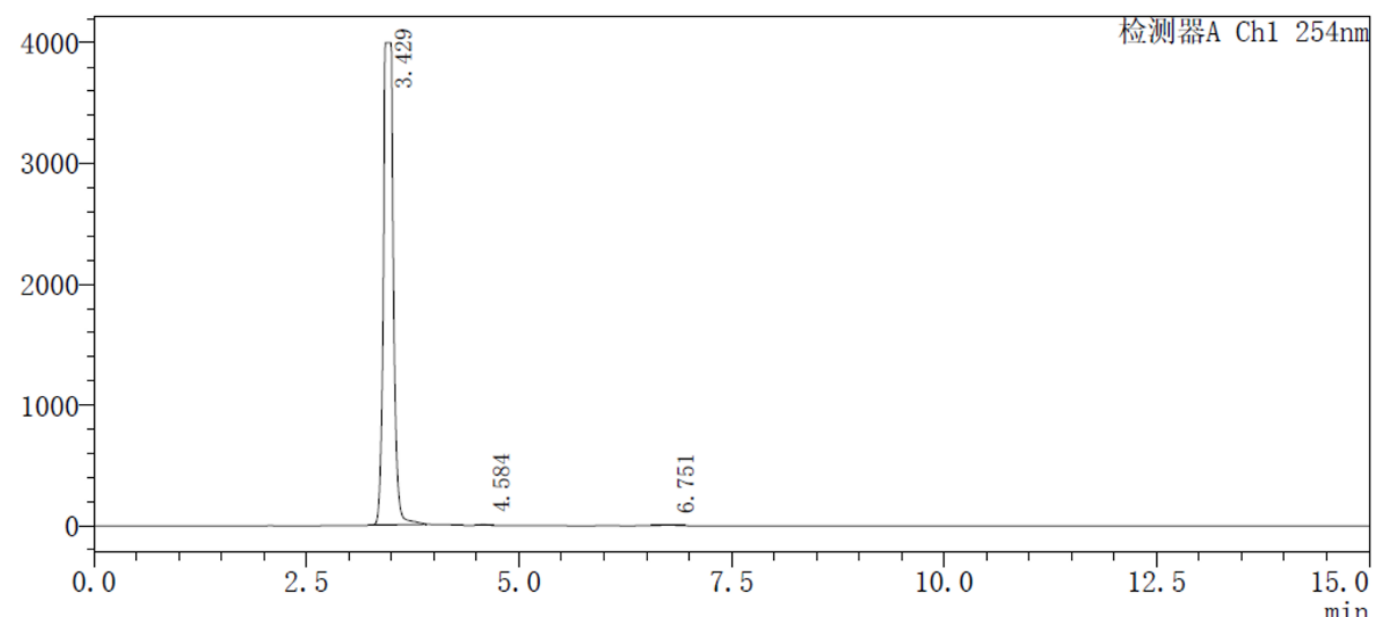

\begin{tabular}{|l|l|l|l|l|}
\hline Peak\# & Retention time & Peak Area & Peak height & Peak Area \% \\
\hline 1 & 3.429 & 32779151 & 3994289 & 99.750 \\
\hline 2 & 4.584 & 24217 & 3959 & 0.074 \\
\hline 3 & 6.751 & 57801 & 4858 & 0.176 \\
\hline In total & & 32861169 & 4003106 & 100 \\
\hline
\end{tabular}




\section{References}

(1) Shao, Y. X.; Huang, M.; Cui, W.; Feng, L. J.; Wu, Y.; Cai, Y.; Li, Z.; Zhu, X.; Liu, P.; Wan, Y.; Ke, H.; Luo, H.-B. Discovery of a Phosphodiesterase 9A Inhibitor as a Potential Hypoglycemic Agent. J. Med. Chem., 2014, 57, 10304-10313.

(2) Wu, D.; Zhang, T.; Chen, Y.; Huang, Y.; Geng, H.; Yu, Y.; Zhang, C.; Lai, Z.; Wu, Y.; Guo, X.; Chen, J.; Luo, H.-B. Discovery and Optimization of Chromeno[2,3-c]pyrrol-9(2H)-ones as Novel Selective and Orally Bioavailable Phosphodiesterase 5 Inhibitors for the Treatment of Pulmonary Arterial Hypertension. J. Med. Chem. 2017, 60, 6622-6637. 